2018-07-27

\title{
Emotion and Sentiment Analysis from Twitter Text
}

Sailunaz, Kashfia

Sailunaz, K. (2018). Emotion and Sentiment Analysis from Twitter Text (Unpublished master's thesis). University of Calgary, Calgary, AB. doi:10.11575/PRISM/32714 http://hdl.handle.net/1880/107533

Downloaded from PRISM Repository, University of Calgary 


\title{
UNIVERSITY OF CALGARY
}

Emotion and Sentiment Analysis from Twitter Text

by

Kashfia Sailunaz

\begin{abstract}
A THESIS
SUBMITTED TO THE FACULTY OF GRADUATE STUDIES

IN PARTIAL FULFILLMENT OF THE REQUIREMENTS FOR THE

DEGREE OF MASTER OF SCIENCE
\end{abstract}

GRADUATE PROGRAM IN COMPUTER SCIENCE

CALGARY, ALBERTA

July, 2018

(C) Kashfia Sailunaz 2018 


\begin{abstract}
Online social networks have emerged as new platform that provide people an arena to share their views and perspectives on different issues and subjects with their friends, family, and other users. We can share our thoughts, mental states, moments and stances on specific social, and political issues through texts, photos, audio/video messages and posts. Indeed, despite the availability of other forms of communication, text is still one of the most common ways of communication in a social network. Twitter was chosen in this research for data collection, experimentation and analysis. The research described in this thesis is to detect and analyze both sentiment and emotion expressed by people through texts in their Twitter posts. Tweets and replies on few recent topics were collected and a dataset was created with text, user, emotion and sentiment information. The customized dataset had user detail like user ID, user name, user's screen name, location, number of tweets/followers/likes/followees. Similarly, for textual information, tweet ID, tweet time, number of likes/replies/retweets, tweet text, reply text and few other text based data were collected. The texts of the dataset were then annotated with proper emotions and sentiments according to some benchmark models. The customized dataset was then used to detect sentiment and emotion from tweets and their replies using machine learning. The influence scores of users were also calculated based on various user-based and tweet-based parameters. Based on those information, both generalized and personalized recommendations were offered for users based on their Twitter activities.
\end{abstract}




\section{Preface}

This thesis is an original work by the author. Few parts of this thesis have been previously published and submitted (in 2018).

Parts of Chapter 1 and 2 were published as "Emotion Detection from Text and Speech: A Survey", in Social Network Analysis and Mining, 8(1), p.28, Springer, 2018[1].

Chapter 3 was published as "Text-Based Analysis of Emotion by Considering Tweets.", in Machine Learning Techniques for Online Social Networks, pp. 219-236, Springer, Cham, $2018[2]$.

Parts of Chapter 1, 2, 4, 5 and 6 were submitted as "Emotion and Sentiment Analysis from Twitter Text", in PLOS ONE, 2018. 


\section{Acknowledgements}

I would like to express my sincere gratitude to my thesis supervisor Dr. Reda Alhajj of the Department of Computer Science at the University of Calgary. His intellectual support and guidelines helped me at every phase of my research work. His participation, suggestions, validations and reviews on every little detail made this research successful. He has been providing unfailing support and continuous encouragement throughout my years of study and through the process of researching and writing this thesis. This accomplishment would not have been possible without him.

I would also like to express my profound gratitude to Dr. Jon Rokne of the Department of Computer Science at the University of Calgary. His continuous support, motivation, encouragement, patience, observation and comments on every step of the research and writing taught me to address and analyze research problems from various perspectives.

I would also like to thank my fellow graduate students at the 'Advanced Database Systems and Applications Lab' of the Department of Computer Science at the University of Calgary. Their friendship, support and motivation helped me coping with my struggles as an international graduate student in a foreign country.

Last but not the least, I would like to thank my parents for supporting me throughout my academic life and being there for me at every step of my life. 


\section{Table of Contents}

$\begin{array}{ll}\text { Abstract } & \text { ii }\end{array}$

$\begin{array}{lll}\text { Preface } & \text { iii }\end{array}$

Acknowledgements $\quad$ iv

Table of Contents $\quad$ v

List of Figures and Illustrations vii

List of Tables $\quad$ ix

List of Symbols, Abbreviations and Nomenclature $\quad \mathrm{x}$

1 Introduction $\quad 1$

1.1 Emotion and Sentiment . . . . . . . . . . . . . . . . 2

1.2 Emotion Analysis and Sentiment Analysis . . . . . . . . . . . . . . . 3

1.3 Emotion and Sentiment in Text . . . . . . . . . . . . . . . . . . . . 4

1.4 Emotion and Sentiment in Twitter Texts . . . . . . . . . . . . . 5

2 Literature Review $\quad 9$

2.1 Emotion Analysis . . . . . . . . . . . . . . . . . . . . 9

2.1.1 Evolution of Emotion . . . . . . . . . . . . . . . . . . . . . . . . . . . . . .

2.1.2 Emotion Models . . . . . . . . . . . . . . . . . . 11

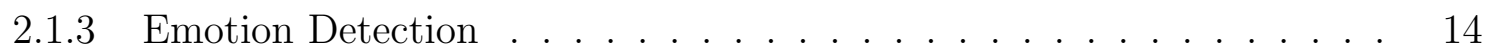

2.2 Emotion Analysis from Text . . . . . . . . . . . . . . . . . 14

2.2.1 Emotion and Sentiment Detection Methods . . . . . . . . . . . . 32

2.2 .2 Datasets . . . . . . . . . . . . . . . . . 34

2.2.3 Influential Users in Social Network . . . . . . . . . . . . . . . . . . . 35

2.3 Few Challenges or Open Problems . . . . . . . . . . . . . . . 38

2.3.1 Text-based Emotion Network Detection . . . . . . . . . . . . . . . 38

2.3.2 Emotion Intensity Detection . . . . . . . . . . . . . . . . . . . . 38

2.3.3 Sarcasm Detection . . . . . . . . . . . . . . . . . . 39

2.3.4 Multiple Emotion Detection . . . . . . . . . . . . . . . . . . 39

2.3.5 Emotion-Cause Detection .................. 39

2.3.6 Personality or Mood Detection . . . . . . . . . . . . . 40 
2.3.7 Emotion versus Individual or Social Parameters . . . . . . . . . . . 40

3 Text-Based Analysis of Emotion by Considering Tweets 42

3.1 Tweet Emotion Detection . . . . . . . . . . . . . . . . . . 42

$3.1 .1 \quad$ Preprocessing . . . . . . . . . . . . . . . . . . . . . . 43

3.1 .2 POS Tagging . . . . . . . . . . . . . . . . . . . . . . . 43

3.1 .3 Emotion Dictionary . . . . . . . . . . . . . . . . . . . . . 44

3.1.4 Unigram Selection and Dataset Annotation . . . . . . . . . . . . 45

3.1.5 Emotion Classification . . . . . . . . . . . . . . . . . 46

3.2 Experimental Results . . . . . . . . . . . . . . . . . . . . . . . 46

3.2 .1 Dataset . . . . . . . . . . . . . . . . . . 47

3.2 .2 Results . . . . . . . . . . . . . . . . . . . . . . . . . 47

3.3 Conclusion . . . . . . . . . . . . . . . . . . . . 55

4 Emotion Detection : From Data Collection to Recommendation 56

4.1 Data Collection . . . . . . . . . . . . . . . . . . . 57

4.2 Pre-processing . . . . . . . . . . . . . . . . . . . . . . . . . . . . . . . . . . 59

4.3 Emotion Detection . . . . . . . . . . . . . . . . . 63

4.4 Influential Users Detection . . . . . . . . . . . . . . . . . . . . 64

4.5 Recommendation . . . . . . . . . . . . . . . 68

5 Experimental Results $\quad 71$

5.1 Emotion and Sentiment Classification . . . . . . . . . . . . . . 71

5.2 Influence Score . . . . . . . . . . . . . . . . . . . . . . . . . . 72

5.3 Recommendations . . . . . . . . . . . . . . . . . . . . . 74

5.4 Networks between Users . . . . . . . . . . . . . . . . . . . . 75

6 Conclusion and Future Works $\quad 82$

6.1 Conclusion . . . . . . . . . . . . . . . . . . . . . . . 82

6.2 Future Works . . . . . . . . . . . . . . . . . . . . . . . . 85

$\begin{array}{ll}\text { Bibliography } & 87\end{array}$ 


\section{List of Figures and Illustrations}

1.1 Sample Tweet and Replies. . . . . . . . . . . . . . . . . . . 6

2.1 Evolution of Emotion in Various Fields . . . . . . . . . . . . . . . . . . 10

2.2 Emotion Models . . . . . . . . . . . . . . . . . . . . . . . . . . . . 13

3.1 Plutchik's Wheel of Emotion. . . . . . . . . . . . . . . . . . 45

3.2 Emotion Network. . . . . . . . . . . . . . . . . . . . . . . . . . . 48

3.3 Gender Vs. Emotion. . . . . . . . . . . . . . . . . . . . . . . . . . . . . 49

3.4 Time Vs. Emotion. . . . . . . . . . . . . . . . . . . . . . 50

3.5 Location Vs. Emotion - Map. . . . . . . . . . . . . . . . . . 51

3.6 Location Vs. Emotion - Pie Chart 1. . . . . . . . . . . . . . . . . . 52

3.7 Location Vs. Emotion - Pie Chart 2. . . . . . . . . . . . . . . 53

3.8 Location Vs. Emotion - Pie Chart 3. . . . . . . . . . . . . . . . . . 54

4.1 Dataset Creation Workflow. . . . . . . . . . . . . . . . . 60

4.2 Preprocessing Workflow. . . . . . . . . . . . . . . . . . . . . . 61

4.3 Sample Tweet Dataset. . . . . . . . . . . . . . . . . . . . . . . . . . 61

4.4 Sample Reply Dataset. . . . . . . . . . . . . . . . . . . . . . . . . . 61

4.5 Sample Retweet Dataset. . . . . . . . . . . . . . . . . . . . . . . . . . 62

4.6 Sample User Dataset. . . . . . . . . . . . . . . . . . . . . . . . . . . . . . 62

4.7 Classification \& Recommender System Workflow. . . . . . . . . . . . . . 63

4.8 Connections between a Tweeter and the Retweeters and Repliers. . . . . . . 65

4.9 Sentiments of Nodes. . . . . . . . . . . . . . . . . . . . . . . . . 65

4.10 Emotions of Nodes. . . . . . . . . . . . . . . . . . . 66

4.11 Suggestions of Connections between Similar Emotion Nodes. . . . . . . . . 66

4.12 Pairwise Comparison Scale in AHP. . . . . . . . . . . . . . . . . 66

4.13 Recommender System Workflow. . . . . . . . . . . . . . . . . . . . 69

5.1 Comparison Matrices for AHP. . . . . . . . . . . . . . . . . . 73

5.2 Users and their Influence Scores. . . . . . . . . . . . . . . . . . . . 74

5.3 General Recommendation according to Sentiment. . . . . . . . . . . . . . 75

5.4 General Recommendation according to Emotion. . . . . . . . . . . . . . . 76

5.5 Personalized Recommendation. . . . . . . . . . . . . . . . . 76

5.6 Retweet Network based on Degree for \#Oscars2018. . . . . . . . . . . . . 77

5.7 Retweet Network for \#Oscars2018. . . . . . . . . . . . . . . . . . . . . . . 78

5.8 Reply Network for \#Oscars2018. . . . . . . . . . . . . . . . . . . . . . 79 
5.9 Tweet Sentiment Network for \#Oscars2018. . . . . . . . . . . . . . . . . . 80

5.10 Tweet Emotion Network for \#Oscars2018. . . . . . . . . . . . . . . . . . 80 


\section{List of Tables}

2.1 Emotion Models. . . . . . . . . . . . . . . . . . . . . . . . . . . . 12

2.2 Related Works with Features and Limitations - Table I. . . . . . . . . . . . 16

2.3 Related Works with Features and Limitations - Table II. . . . . . . . . . . 17

2.4 Related Works with Features and Limitations - Table III. . . . . . . . . . . . 18

2.5 Related Works with Features and Limitations - Table IV. . . . . . . . . . . . 19

2.6 Related Works with Features and Limitations - Table V. . . . . . . . . . . . 20

2.7 Related Works with Features and Limitations - Table VI. . . . . . . . . . . . 21

2.8 Related Works with Features and Limitations - Table VII. . . . . . . . . . . 22

2.9 Related Works with Features and Limitations - Table VIII. . . . . . . . . . . 23

2.10 Related Works with Features and Limitations - Table IX. . . . . . . . . . . . 24

2.11 Related Works with Features and Limitations - Table X. . . . . . . . . . . 25

2.12 Related Works with Features and Limitations - Table XI. . . . . . . . . . . . 26

2.13 Features used in Existing Works - Table I. . . . . . . . . . . . . . . . . 27

2.14 Features used in Existing Works - Table II. . . . . . . . . . . . . . . . . 28

2.15 Features used in Existing Works - Table III. . . . . . . . . . . . . . . . . . 29

2.16 Features used in Existing Works - Table IV. . . . . . . . . . . . . . . . . 30

2.17 Features used in Existing Works - Table V. . . . . . . . . . . . . . . 31

2.18 Datasets . . . . . . . . . . . . . . . . . . . . . . . . 35

4.1 Tweet Attributes . . . . . . . . . . . . . . . . . . . . . . . . . . 59

4.2 User Attributes . . . . . . . . . . . . . . . . . . . . . . . . . . . . . 59

5.1 Sentiment and Emotion Classification Accuracy (in percentage - \%) . . . . 73 


\section{List of Symbols, Abbreviations and Nomenclature}

\begin{tabular}{ll} 
Symbol or abbreviation & Definition \\
e.g. & exempli gratia \\
i.e. & id est \\
etc. & et cetera \\
et al. & et alia \\
HCI & Human Computer Interaction \\
EEG & Electroencephalography \\
OCC & Ortony, Clore and Collins \\
SVM & Support Vector Machine \\
NLP & Natural Language Processing \\
LSTM & Long Short Term Memory \\
POS & Parts of Speech \\
BWS & Best Worst Scaling \\
ETCC & Emotion Tweet Corpus for Classification \\
ETCR & Emotion Tweet Corpus for Relevance \\
CNN & Convolutional Neural Network \\
NN & Neural Network \\
\hline
\end{tabular}


NAVA

PMI

BTM

LIWC

NRC

ESN

ISEAR

T-PICE

AAI

URL

AHP

KNN

API

ID

HDD

MIN

MAX

IS

$\mathrm{IS}_{U}$

$\mathrm{IS}_{T}$

$\mathrm{TS}_{U}$
Noun Adjective Verb Adverb

Pointwise Mutual Information

Biterm Topic Model

Linguistic Inquiry and Word Count

National Research Council Canada

EmoSenticNet

International Survey on Emotion

Antecedents and Reactions

Twitter Personality based Influential Communities Extraction

Acquaintance Affinity Identification

Uniform Resource Locator

Analytic Hierarchy Process

K Nearest Neighbor

Application Programming Interface

Identity Document

Hidden Markov Model

Minimum

Maximum

Influence Score

Influence Score based on

User Parameters

Influence Score based on

Tweet Parameters

Total Number of Tweets Posted

by the User 


\begin{tabular}{|c|c|}
\hline $\mathrm{W}_{T}$ & Weight Assigned to $\mathrm{TS}_{U}$ \\
\hline $\mathrm{FS}_{U}$ & $\begin{array}{l}\text { Total Number of Followers of } \\
\text { the User }\end{array}$ \\
\hline $\mathrm{W}_{F}$ & Weight Assigned to $\mathrm{FS}_{U}$ \\
\hline $\mathrm{OS}_{U}$ & $\begin{array}{l}\text { Total Number of Followees of } \\
\text { the User }\end{array}$ \\
\hline $\mathrm{W}_{O}$ & Weight Assigned to $\mathrm{OS}_{U}$ \\
\hline $\mathrm{LS}_{U}$ & $\begin{array}{l}\text { Total Number of Likes of } \\
\text { the User }\end{array}$ \\
\hline $\mathrm{W}_{L}$ & Weight Assigned to $\mathrm{LS}_{U}$ \\
\hline $\mathrm{RS}_{T}$ & $\begin{array}{l}\text { Total Number of Times a } \\
\text { Tweet was Retweeted }\end{array}$ \\
\hline $\mathrm{W}_{R}$ & Weight Assigned to $\mathrm{RS}_{T}$ \\
\hline $\mathrm{CS}_{T}$ & $\begin{array}{l}\text { Total Number of Replies or } \\
\text { Comments of a Tweet }\end{array}$ \\
\hline $\mathrm{W}_{C}$ & Weight Assigned to $\mathrm{CS}_{T}$ \\
\hline $\mathrm{PS}_{T}$ & $\begin{array}{l}\text { Total Number of Likes } \\
\text { of a Tweet }\end{array}$ \\
\hline $\mathrm{W}_{P}$ & Weight Assigned to $\mathrm{PS}_{T}$ \\
\hline $\mathrm{AS}_{T}$ & $\begin{array}{l}\text { Agreement Score of the } \\
\text { Reply to the Tweet }\end{array}$ \\
\hline $\mathrm{W}_{A}$ & Weight Assigned to $\mathrm{AS}_{T}$ \\
\hline $\mathrm{SS}_{T}$ & $\begin{array}{l}\text { Sentiment Score of } \\
\text { a Tweet or a Reply }\end{array}$ \\
\hline $\mathrm{W}_{S}$ & Weight Assigned to $\mathrm{SS}_{T}$ \\
\hline $\mathrm{ES}_{T}$ & $\begin{array}{l}\text { Emotion Score of } \\
\text { a Tweet or a Reply }\end{array}$ \\
\hline $\mathrm{W}_{E}$ & Weight Assigned to $\mathrm{ES}_{T}$ \\
\hline
\end{tabular}




\section{Chapter 1}

\section{Introduction}

Emotion recognition is the process of identifying human emotion by merely depending on personal skills and interpretation of interpersonal interaction, by automating the process with a computer-based system, or by a semi-automated approach combining both. Many methodologies have been developed for automatic emotion recognition, e.g., human computer interaction (HCI) [3, 4, 5] which targets making HCI more natural[6]. There are several intelligent assistants, such as Siri[7] and Cortana[8] that use many quirks to make the interactions with human users more natural. Emotion recognition can further improve HCI by allowing the system to consider emotions before performing tasks.

Recognizing human emotions accurately is a laborious task due to the versatility and ambiguity of emotions. Same emotions may be expressed in multiple ways, and multiple emotions sometimes have the same expression. Emotions may vary based on personality, gender, location, ethnicity, culture, situation, in addition to many other psychological, social and individual parameters. Sometimes, detecting the actual emotion of a person from a piece of text, his/her speech or facial expressions is difficult even for another human. When it comes to automatic emotion detection by a computer, the level of complication of the problem is easily imaginable. A large number of researchers have been working on emotion 
detection from various types of inputs( e.g., text, image, audio, video, etc.). In other words, emotion recognition can be performed based on different sources of information, such as $\operatorname{speech}[9,10,11], \operatorname{text}[12,13,14,15]$, or video/image[16, 17, 18]. Several existing systems use combination of these information for emotion recognition[19, 20]. However, in many cases, not all textual, audio or visual information are available. For example, some online retailers like Amazon[21] might perform emotion recognition through text, since they have text reviews data available; call centers might use audio speech recognition for emotion detection[22]; while emotion recognition for security cameras will have to rely on visual information only. The focus of this research is emotion and sentiment detection and analysis from text. Although for text based analysis, the input text can be any social media posts, comments, reviews, or any other form of text; Twitter texts were used for analysis and experiments.

\subsection{Emotion and Sentiment}

'Emotion' detection from text is comparatively more complex task than 'sentiment' detection. Although the terms 'emotion' and 'sentiment' are considered synonymous, they represent two different concepts in text analysis[23]. According to Oxford Dictionary, 'emotion' is "a strong feeling deriving from one's circumstances, mood, or relationships with others", whereas 'sentiment' is "a view or opinion that is held or expressed" [24]. Cambridge Dictionary defines 'emotion' as "a strong feeling such as love or anger, or strong feelings in general" and 'sentiment' as "a thought, opinion, or idea based on a feeling about a situation, or a way of thinking about something" [25]. The American Psychological Association defines 'emotion' as "a complex pattern of changes, including physiological arousal, feelings, cognitive pro-

cesses, and behavioral reactions, made in response to a situation perceived to be personally significant" [26]. In general, 'sentiment' is defined as the effect of 'emotion'[27]. 'Happiness', 
'Anger', 'Love' are examples of emotions and 'Positive', 'Negative', 'Negative', respectively, are examples of corresponding sentiments of these emotions. Therefore, 'sentiment' is a more generalized interpretation of 'emotion' and for that reason, the term 'emotion' is mentioned more than 'sentiment' throughout this thesis as sentiment can be derived from emotion.

\subsection{Emotion Analysis and Sentiment Analysis}

The analysis of sentiment and emotion differ like their definitions. 'Sentiment analysis' focuses on identifying and studying subjective information from human sentiments to find out the attitude of a person on some specific topic or the overall context of an event. Similarly, 'emotion analysis' is an effective study of human emotions that tries to identify the proper emotion from context, and analyze it according to some predefined emotion class models. Emotion and sentiment detection from text have their applications in almost every aspect of our daily life, such as, making efficient e-learning systems based on student's emotion; improving human computer interactions; monitoring mental health of people; modifying or improving business strategies according to the emotion of customers; detecting public emotion on any national, international or political event; detecting potential criminals or terrorists by analyzing the emotions of people after a terrorist attack or crime; improving the performances of chatbots and other automatic feedback systems; and so on. For instance, analyzing product reviews to find out the emotions of consumers regarding a product can be used to improve business strategies. Emotions extracted from tweets or other social media posts on different personal or public events can be analyzed for personal or public mood detection. For instance, a person whose posts reflect sadness, frustration and depression may be immediately identified and referred to a specialist before something unpleasant like suicide might happen. Emotion analysis on social media posts about terrorism or terrorists can help law enforcement organizations to detect potential future terrorists. Analyzing the 
emotions of a specific person over time can be an interesting tool for character analysis. Emotion analysis can also be applied in many other business, health, social and political organizations to improve or modify their policies and approaches.

\subsection{Emotion and Sentiment in Text}

Human emotion is a very complicated, multidimensional phenomenon that reflects the personality and behavioral traits of people. In their daily life, people express their emotions on different events, persons, environment and every little thing surrounding them. They use various communication methods to convey their emotions to others. The most common way to reveal emotions to other people is through speech and facial expressions. But, with the rise of social networks, people now capture and express their emotions more frequently and comfortably through their social media activities. Although the advancement of technology allows the users of social network to demonstrate their emotion by 'audios' and 'videos', 'text' is still the most common form of communication in social networking platforms. People profess their emotions and sentiments via their social network posts (e.g. status, comments, blogs, microblogs). In recent textual emotion analysis studies, social network posts have been used for text emotion analysis due to the huge number of participants and posts. Almost 2.46 billion people are active in various social network and are members of one or more social networks like Facebook, Twitter, Instagram, Youtube[28]. Analyzing these texts and detecting emotion from their words and semantics is quite challenging. Emotion and sentiment detection from text has been a promising research topic over the years and considerable efforts have been invested in trying to build a perfect automated system capable of detecting correct human emotion from text.

Researchers in various fields (e.g. psychology, business, computer science, affective com- 
puting, artificial intelligence) have been working on detecting and analyzing emotions from a piece of text. Various methods were applied to detect the specific emotion from texts. Due to the complexity of human emotions, the process has lots of challenges yet to address and overcome. One small paragraph can contain multiple emotions with different polarities (positive or negative sentiments). Sometimes, emotions are not even expressed explicitly; a paragraph may direct to one or several subtle emotions that are implicit and therefore more difficult to detect.

\subsection{Emotion and Sentiment in Twitter Texts}

Emotion detection from tweets is a comparatively new field compared to sentiment analysis[29] using micro-blogs or tweets. Twitter[30] is a social networking website created in 2006 and became very popular because of the lack of restrictions and frequencies of posts. Posts are called tweets and the maximum length of a tweet is only 140 characters. Twitter data is a popular choice for text analysis tasks because of the limited amount characters allowed and the global use of Twitter to express opinions on different issues among people of all ages. Extracting text from Twitter and analyzing tweets posted by people may give an idea of their emotional states and the reasons behind those emotions. The nature of Twitter data and the versatility of human emotions make it difficult for automatic emotion detection from tweets. Sentiment detection, on the other hand, is a relatively easier process.

In this thesis, Twitter network was analyzed for emotion and sentiment detection and analysis. The emotions and sentiments were detected from tweets and their replies and an emotion network was formed based on the texts posted by the users. From the emotion network, the influential people for all types of emotions and sentiments were detected. Finally, a trust network was computed based on the emotion similarities and influences. Figure 1.1 


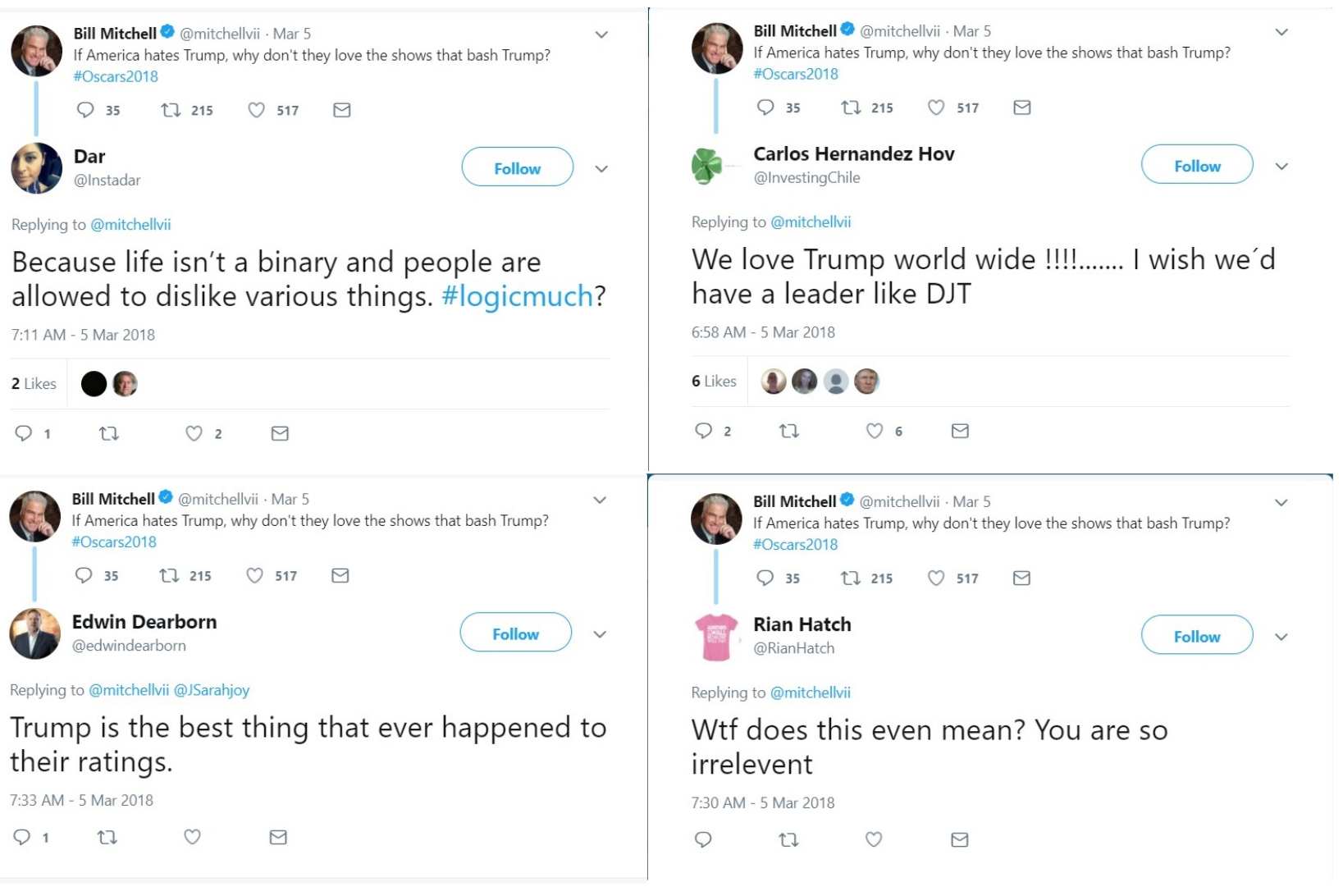

Figure 1.1: Sample Tweet and Replies.

shows an example of a sample tweet and various types of replies to the tweet. The author of the tweet expressed his surprise on an issue and some people replied agreeing to it like the second and third replies. Some people disagreed to the tweet and showed disgust or anger like the fourth reply. Some replies like the first one tried to answer to the tweet with logic and some other replies expressed no particular emotions at all.

As no existing Twitter dataset were found consisting both tweets and their replies, the texts from tweets and replies on specific recent topics were collected to create a customized dataset. Both text-based and user-based data were collected for the dataset. After passing them through few preprocessing and cleaning phases, a structured dataset was generated. All texts were annotated according to their emotions and sentiments. The annotated datasets were then used to train and test separate Naive Bayes classifiers for supervised classification 
of emotions and sentiments. The text-based parameters were then merged with user-based parameters to detect the influential users of the emotion and sentiment networks. The recommender system used k-means clustering to create clusters of users based on their emotions and sentiments individually. Finally, the recommender system used the influence scores of users to provide generalized and personalized recommendation for users. To the best of my knowledge, no existing research works used the replies to a tweet to generate emotion and sentiment network, and applied emotion, sentiment and agreement based scores to calculate influence scores of users in a network.

The novelty of this research is the inclusion of not only the tweets but also the replies of the tweets. To the best of my knowledge, no existing works considered the texts of the replies of tweets. Hence, starting from the creation of the dataset which included the reply texts, including few new parameters with existing parameters for influential user detection and recommendation generation were the unique contributions of this research. Adding three new parameters like agreement score, emotion score and sentiment score based on the texts of tweets and replies in the calculation of influence scores, the calculated influence scores and generated recommendations were more precised to the emotion and sentiment of users on particular topics. In other words, the research problem addressed in this thesis was the inclusion of some emotion and sentiment based parameters with numerical parameters of tweets and users to form an emotion and sentiment based network within the Twitter network. For intermediate methods and calculations, some existing approaches were used. For example, Naive Bayes classifiers were used for emotion and sentiment classification and k-means clustering was used to create sentiment and emotion clusters of users.

The rest of this thesis is outlined as follows. Some related works are summarized in Chapter 2. Chapter 3 explains an emotion based analysis on tweets. Chapter 4 describes the methodology of the system including dataset generation, emotion and sentiment de- 
tection, influence score calculations and recommendation generation with the frameworks and processes used. The datasets, features and methodologies used in Chapter 3 and 4 were completely different. Chapter 3 represents an analysis of a twitter dataset including emotion detection and the variations between emotions and other parameters like gender of the user, tweet collection time and location of the user. On the other hand, chapter 4 represents the methodology of creating another twitter dataset with different parameters and then analyzing the dataset by finding influential users of a network. Chapter 5 shows experimental results and analysis of the results based on the dataset and methodology of chapter 4. Chapter 6 concludes the thesis and outlines some possible future directions. As some parts of this thesis have already been published, the minor overlaps between chapters are keeping the flow of the content of this thesis by connecting the chapters with one another. In this thesis, most of the chapters has distinct methodology and independent testing. 


\section{Chapter 2}

\section{Literature Review}

\subsection{Emotion Analysis}

Human beings express emotions in multidimensional ways. Some common ways are through their writing, their speech, their facial expression, their body language and their gesture. According to previous researches, emotion and sentiment evolve over time and circumstances. These emotions can be categorized with different emotion models defining the set of emotions from various perspectives. This chapter summarizes the research areas and contributions of recent researchers on detection and analysis of human emotion and sentiment from texts.

\subsubsection{Evolution of Emotion}

In 1872, after completing some psychological experiments on facial expressions captured in different circumstances on both humans and animals, Charles Darwin claimed that human and other animals express emotions with similar expressions and behavior under similar circumstances[31]. His theory of emotion was also related to era and associated circumstances. According to his claims, emotions in human and other animals are realized over time. He discussed about general principles of emotions in detail; means of expressions of 
emotions in both man and animals; causes and effects of all possible emotions like anxiety, grief, dejection, despair, joy, love, devotion, etc.; explanation of emotions with images to show the expressions for particular emotions. Darwin claimed that some emotional expressions are universal for people all over the world. He also claimed that not only humans, but also animals of similar species react similarly to a situation. His experiments revealed that for some emotions similar expressions exist even in species which are not very similar. Some philosophical and spiritual categorization of emotions were present before that as described in $[32,33]$.

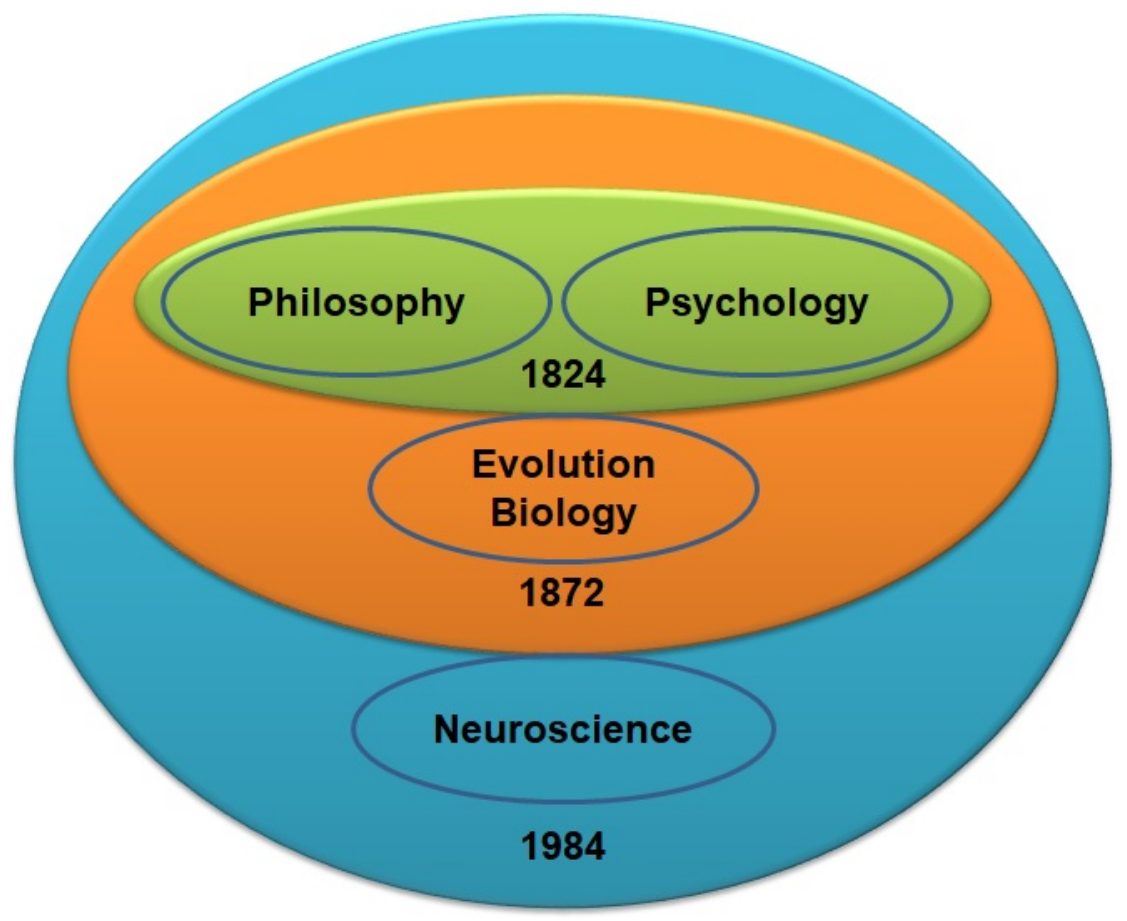

Figure 2.1: Evolution of Emotion in Various Fields

The study of emotions started as a branch of philosophy and psychology theories. Darwin claimed that emotions and their expressions are also related to biological causes. Later, emotions were described as "brain mechanisms that are outputs of functional properties of neural systems" [34]. Figure 2.1 shows the evolution of emotion in different fields of studies. 
According to the evolution theory, it was found that different human emotions were realized at different phases of human life in various eras. Human emotions were defined, explained, categorized and analyzed by psychologists, sociologists, neuroscientists, biologists and researchers from many other fields over time, hence various emotion models emerged to cover all possible human emotions.

\subsubsection{Emotion Models}

From psychological point of view, human emotions can be identified and grouped based on emotion type, emotion intensity, and many other parameters, which can be all combined and realized into emotion models. Emotion models are structured form or way of defining various human emotions according to some scores, ranks or dimensions. Existing emotion models define various kinds of emotions according to duration, intensity, synchronization, rapidity of change, event focus, appraisal elicitation, and behavioral impact[35].

Based on different emotion theories, existing emotion models can be divided into two classes - 'Categorical' and 'Dimensional'[12]. Categorical emotion models define a list of categories of emotions which are discrete from each others. On the other hand, Dimensional emotion models define a few dimensions with some parameters and specify emotions according to those dimensions. Two or three dimensions are used in most dimensional emotion models - 'valence' (indicates the positivity or negativity of an emotion), 'arousal' (indicates the excitement level of an emotion) and 'dominance' (indicates the level of control over an emotion) $[36,37]$. 


\begin{tabular}{|c|c|c|c|}
\hline Model & Emotions & Approach & Structure \\
\hline Ekman[38] & "Anger, Disgust, Fear, Joy, Sadness, Surprise & Categorical & - \\
\hline Shaver[39] & Anger, Fear, Joy, Love, Sadness, Surprise & Categorical & Tree \\
\hline Oatley[40] & Anger, Anxiety, Disgust, Happiness, Sadness & Categorical & - \\
\hline Plutchik[41] & $\begin{array}{c}\text { Acceptance, Admiration, Aggressiveness, } \\
\text { Amazement, Anger, Annoyance, } \\
\text { Anticipation, Apprehension, Awe, } \\
\text { Boredom, Contempt, Disapproval, } \\
\text { Disgust, Distraction, Ecstasy, } \\
\text { Fear, Grief, Interest, } \\
\text { Joy, Loathing, Love, } \\
\text { Optimism, Pensiveness, Rage, } \\
\text { Remorse, Sadness, Serenity, } \\
\text { Submission, Surprise, Terror, } \\
\text { Trust, Vigilance }\end{array}$ & Dimensional & Wheel \\
\hline Circumplex $[42]$ & $\begin{array}{c}\text { Afraid, Alarmed, Angry, } \\
\text { Annoyed, Aroused, Astonished, } \\
\text { At ease, Bored, Calm, Content, } \\
\text { Delighted, Depressed, Distressed, } \\
\text { Droopy, Excited, Frustrated, } \\
\text { Glad, Gloomy, Happy, } \\
\text { Miserable, Pleased, Relaxed, } \\
\text { Sad, Satisfied, Serene, } \\
\text { Sleepy, Tense, Tired }\end{array}$ & Dimensional & $\begin{array}{l}\text { Valence, } \\
\text { Arousal }\end{array}$ \\
\hline $\mathrm{OCC}[43]$ & $\begin{array}{l}\text { Admiration, Anger, Appreciation, } \\
\text { Disappointment, Disliking, Fear, } \\
\text { Fears-confirmed, Gloating, } \\
\text { Gratification, Gratitude, Happy-for, } \\
\text { Hope, Liking, Pity, Pride, } \\
\text { Sorry-for, Relief, Remorse, Reproach, } \\
\text { Resentment, Self-Reproach, Shame }\end{array}$ & Dimensional & Tree \\
\hline Lovheim $[44]$ & $\begin{array}{c}\text { Anger/Rage, Contempt/Disgust, } \\
\text { Distress/Anguish, Enjoyment/Joy, } \\
\text { Fear/Terror, Interest/Excitement, } \\
\text { Shame/Humiliation, Surprise/Startle }\end{array}$ & Dimensional & Cube \\
\hline
\end{tabular}

Table 2.1: Emotion Models. 

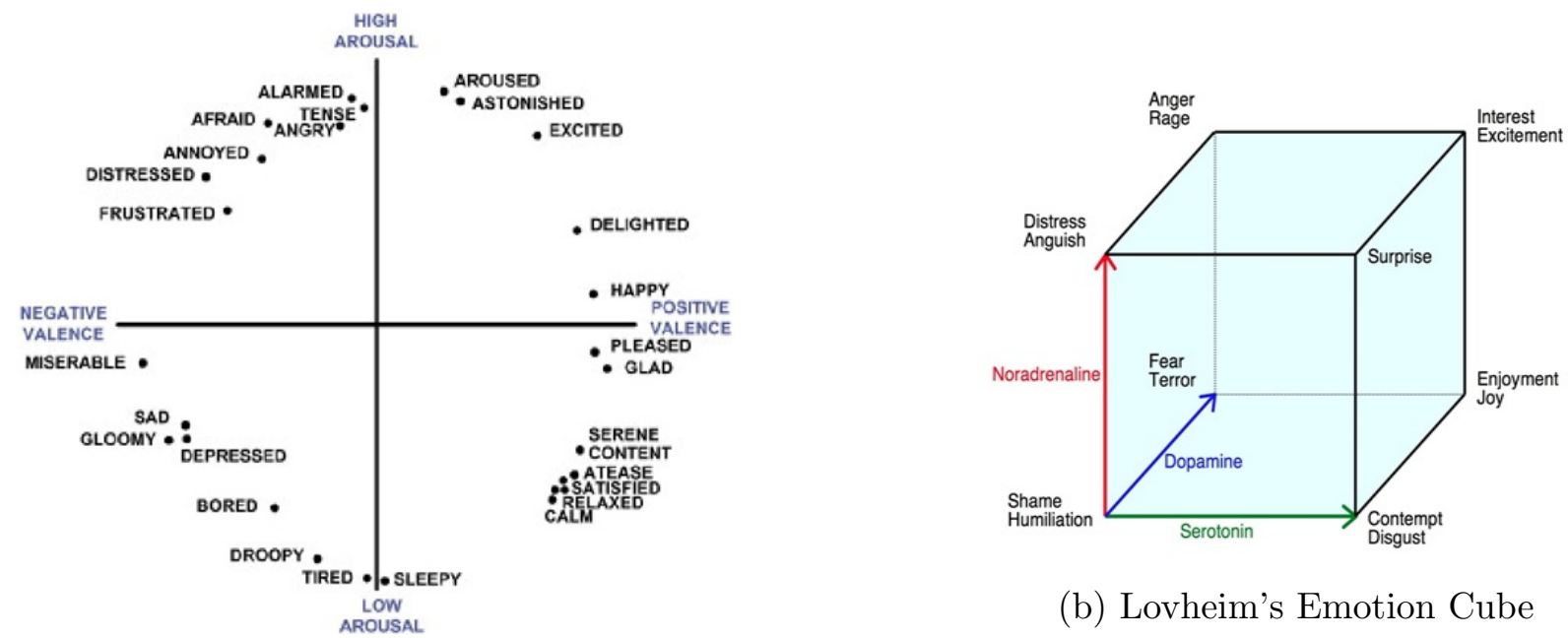

(b) Lovheim's Emotion Cube

(a) Circumplex Emotion Model

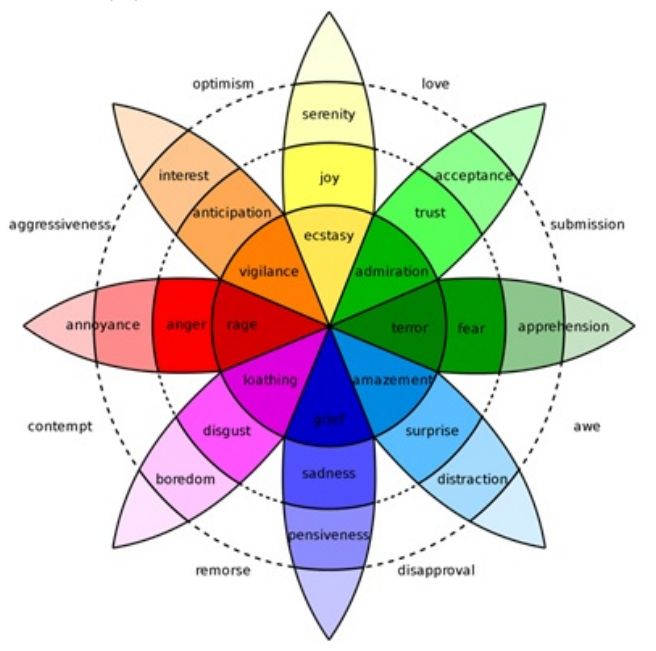

(c) Plutchik's Emotion Wheel

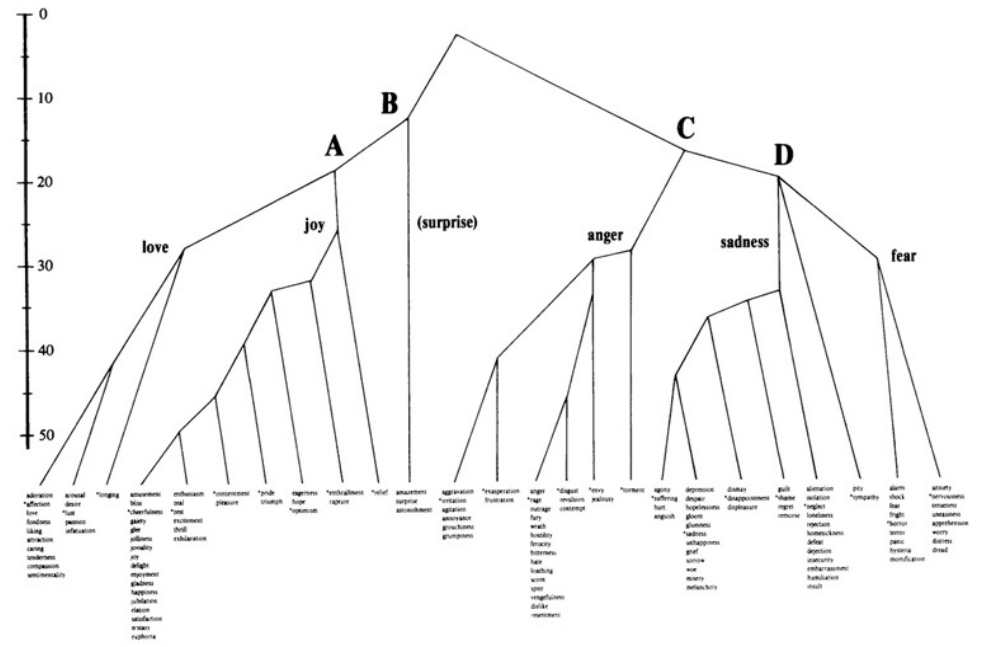

(d) Shaver's emotion model

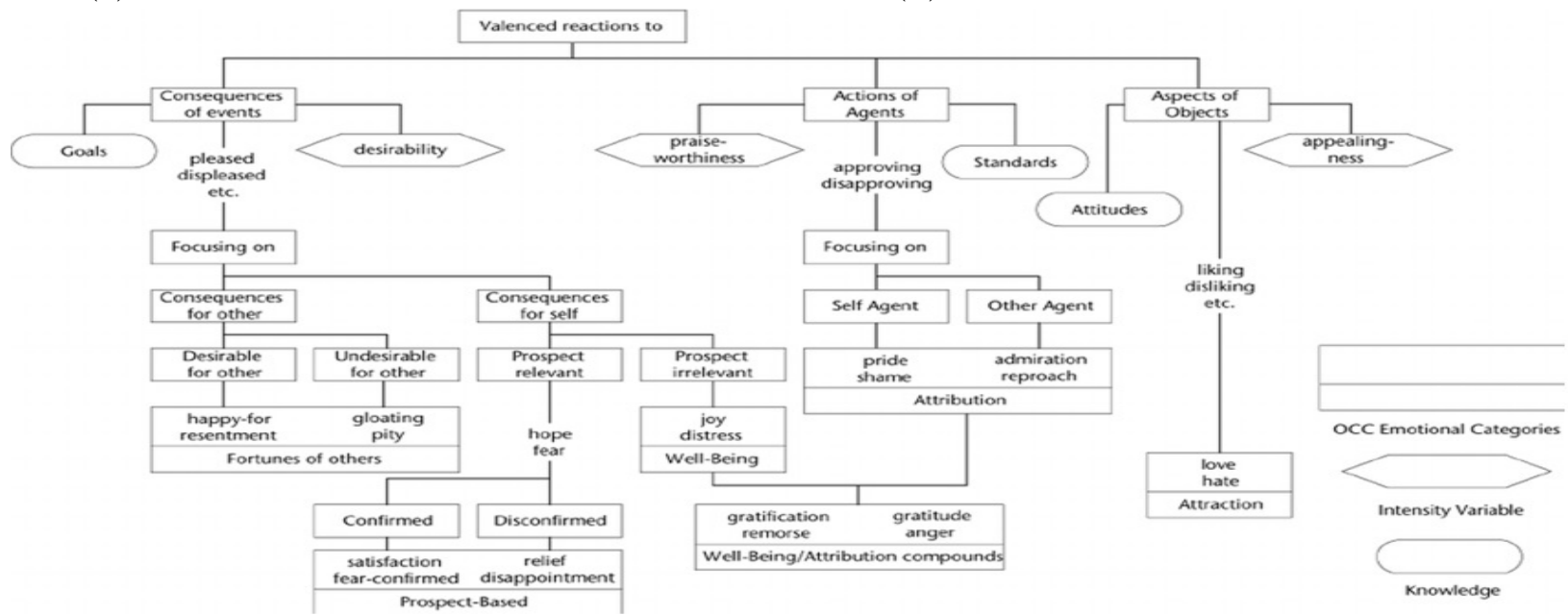

(e) OCC Emotion Model

Figure 2.2: Emotion Models 
Table 2.1 summarizes few basic emotion models used frequently in the literature and express almost all possible human emotions. The table lists the emotions for each model and the dimensions for dimensional emotion models. Figure 2.2 shows some emotion models that are mostly used in emotion based research.

\subsubsection{Emotion Detection}

Emotion extraction from different types of social network components is a research topic which is being investigated for a long time now. Various kinds of contents posted by people on social networking platforms have been analyzed to detect emotions behind the posts. In some works, any combination of voice tone, speech, facial expressions, gestures, EEG signals, different types of bio signals and texts were used to detect emotion from multimodal data $[45,46,47]$. On the other hand, most of the works on emotion detection focus on one specific type of content. Speech, voice tone and frequency from audios were used as inputs for emotion detection in[48, 49], whereas facial expressions and gestures from videos were used to extract emotions in[50]. But as mentioned earlier, this thesis will focus on emotion and sentiment detection from text.

\subsection{Emotion Analysis from Text}

In the present era of smart phones and social networking platforms, a number of people prefer sharing their emotions, sentiments and achievements via audio or video files because of the real time features of these components. However, even if the popularity of these interactive contents is increasing, most people still turn to text for their communication and interaction in daily life as well as on social networking platforms. 
Text is still the primary choice for people to express their feelings towards other persons, events or things. Extracting emotion from text is still more difficult because of the nature of the data. Detecting emotions from text is easier if words representing a particular emotion were explicit in the text. But most of the time, emotion is expressed in a subtle way. Again, sometimes, multiple emotions exist in a single piece of text. Some texts has emotions and words which are ambiguous, some words have multiple meanings, and multiple words mean the same emotion. Some text represents sarcasm, or use slangs. Multilingual text, spelling mistakes, acronyms, grammatically incorrect sentences are some common characteristics of texts available online. These limitations of textual data sometimes make automatic emotion detection nearly impossible. Research on emotion extraction from text is a very popular topic. Modifications, improvements and new approaches to address challenges of this task is attracting the attention of researchers all over the world. Some existing surveys on text emotion detection and analysis summarize the works done in this field from different timelines describing existing emotion detection methods, approaches, datasets, experiments and outcomes[51, 52, 53, 1]. Some researchers proposed their own approach with a detailed survey on textual emotion $[54,55,56]$. In Table $2.2-2.12$, some of the existing works and their contributions in this field are listed. The works from the last ten years (2008-2017) were included in this summary. 


\begin{tabular}{|c|c|c|c|c|c|c|}
\hline Authors & Type & Approach & Method & Emotions & Features & Limitations \\
\hline $\begin{array}{l}\text { Yadollahi } \\
\text { et al.[51] }\end{array}$ & Survey & - & $\begin{array}{l}\text { Existing } \\
\text { emotion } \\
\text { detection } \\
\text { methods }\end{array}$ & $\begin{array}{l}\text { Basic } \\
\text { Emotion } \\
\text { Theories }\end{array}$ & $\begin{array}{l}\text { Existing Lexicons, } \\
\text { Datasets, Mining } \\
\text { methods for } \\
\text { Twitter, Mining } \\
\text { for English } \\
\text { and other } \\
\text { languages; } \\
\text { Well structured } \\
\text { investigation and } \\
\text { categorization of } \\
\text { emotion mining }\end{array}$ & - \\
\hline $\begin{array}{c}\text { Binali } \\
\text { et al. [54] }\end{array}$ & $\begin{array}{c}\text { Survey } \\
\text { and a } \\
\text { proposed } \\
\text { approach }\end{array}$ & $\begin{array}{l}\text { Hybrid of } \\
\text { keyword } \\
\text { and } \\
\text { learning } \\
\text { based } \\
\text { method }\end{array}$ & $\begin{array}{c}\text { Keyword and } \\
\text { learning } \\
\text { based } \\
\text { methods }\end{array}$ & $\begin{array}{l}\text { Basic } \\
\text { Emotion } \\
\text { Theories }\end{array}$ & $\begin{array}{c}\text { Description of } \\
\text { computational } \\
\text { approaches } \\
\text { for emotion } \\
\text { detection from } \\
\text { text; Improved } \\
\text { emotion detection by } \\
\text { combining semantic } \\
\text { and syntactic } \\
\text { information }\end{array}$ & - \\
\hline $\begin{array}{l}\text { Canales } \\
\text { et al.[37] }\end{array}$ & Survey & - & $\begin{array}{l}\text { Lexicon and } \\
\text { machine- } \\
\text { learning } \\
\text { based } \\
\text { methods }\end{array}$ & $\begin{array}{l}\text { Basic } \\
\text { Emotion } \\
\text { Theories }\end{array}$ & $\begin{array}{c}\text { Collection of } \\
\text { Lexical-based, } \\
\text { Supervised } \\
\text {-learning based } \\
\text { and Unsupervised- } \\
\text { learning based } \\
\text { existing works; } \\
\text { Comparisons } \\
\text { between } \\
\text { lexical and } \\
\text { machine-learning } \\
\text { based approach } \\
\text { and limitations } \\
\text { of existing } \\
\text { systems }\end{array}$ & $\begin{array}{l}\text { Proposed } \\
\text { a new } \\
\text { direction } \\
\text { on deep } \\
\text { analysis } \\
\text { but didn’t } \\
\text { explain } \\
\text { in detail }\end{array}$ \\
\hline
\end{tabular}

Table 2.2: Related Works with Features and Limitations - Table I. 


\begin{tabular}{|c|c|c|c|c|c|c|}
\hline Authors & Type & Approach & Method & Emotions & Features & Limitations \\
\hline $\begin{array}{l}\text { Chopade } \\
\text { et al. [52] }\end{array}$ & Survey & - & $\begin{array}{l}\text { Keyword } \\
\text { Spotting, } \\
\text { Lexical } \\
\text { Affinity, } \\
\text { Learning } \\
\text { Based } \\
\text { and Hybrid } \\
\text { method }\end{array}$ & $\begin{array}{l}\text { Basic } \\
\text { Emotion } \\
\text { Theories }\end{array}$ & $\begin{array}{l}\text { Applications of } \\
\text { textual emotion } \\
\text { detection; } \\
\text { Limitations of } \\
\text { existing } \\
\text { approaches; } \\
\text { Text normalization } \\
\text { techniques }\end{array}$ & - \\
\hline $\begin{array}{l}\text { Tripathi } \\
\text { et al.[53] }\end{array}$ & Survey & - & $\begin{array}{l}\text { Keyword } \\
\text { Spotting, } \\
\text { Lexical } \\
\text { Affinity, } \\
\text { Statistical } \\
\text { NLP }\end{array}$ & $\begin{array}{l}\text { Basic } \\
\text { emotions, } \\
\text { their } \\
\text { properties } \\
\text { represen- } \\
\text { tations } \\
\text { and } \\
\text { models }\end{array}$ & $\begin{array}{l}\text { Emotive potential } \\
\text { of text, Definition } \\
\text { and generation of } \\
\text { Datasets and } \\
\text { Lexicons; } \\
\text { List of existing } \\
\text { textual emotion } \\
\text { detection works; } \\
\text { Emotion analysis } \\
\text { applications; } \\
\text { Possible future } \\
\text { directions }\end{array}$ & - \\
\hline $\begin{array}{c}\text { Kao } \\
\text { et al.[55] }\end{array}$ & $\begin{array}{c}\text { Survey } \\
\text { and a } \\
\text { proposed } \\
\text { approach }\end{array}$ & $\begin{array}{c}\text { Case-based } \\
\text { reasoning } \\
\text { method }\end{array}$ & $\begin{array}{l}\text { Keyword- } \\
\text { based, } \\
\text { machine- } \\
\text { learning } \\
\text { based and } \\
\text { hybrid } \\
\text { methods }\end{array}$ & $\begin{array}{l}22 \text { OCC } \\
\text { emotions }\end{array}$ & $\begin{array}{c}\text { Formally defined } \\
\text { 'emotion detection' } \\
\text { problem, existing } \\
\text { approaches and their } \\
\text { limitations, } \\
\text { possible solutions; } \\
\text { A new approach } \\
\text { with semantic } \\
\text { analysis and } \\
\text { case-based } \\
\text { reasoning }\end{array}$ & $\begin{array}{c}\text { Proposed } \\
\text { method } \\
\text { was not } \\
\text { implem- } \\
\text { ented }\end{array}$ \\
\hline
\end{tabular}

Table 2.3: Related Works with Features and Limitations - Table II. 


\begin{tabular}{|c|c|c|c|c|c|c|}
\hline Authors & Type & Approach & Method & Emotions & Features & Limitations \\
\hline $\begin{array}{l}\text { Shivhare } \\
\text { et al.[56] }\end{array}$ & $\begin{array}{c}\text { Survey } \\
\text { and a } \\
\text { proposed } \\
\text { approach }\end{array}$ & $\begin{array}{c}\text { Emotion } \\
\text { ontology } \\
\text { based } \\
\text { emotion } \\
\text { detector } \\
\text { algorithm }\end{array}$ & $\begin{array}{c}\text { Keyword } \\
\text { Spotting, } \\
\text { Lexical } \\
\text { Affinity, } \\
\text { Learning } \\
\text { Based } \\
\text { and Hybrid } \\
\text { method }\end{array}$ & - & $\begin{array}{c}\text { Discussions on } \\
\text { different emotion } \\
\text { detection } \\
\text { techniques, } \\
\text { their limitations; } \\
\text { Design of a new } \\
\text { ontology based } \\
\text { algorithm }\end{array}$ & $\begin{array}{l}\text { Proposed } \\
\text { method } \\
\text { was not } \\
\text { implem- } \\
\text { ented }\end{array}$ \\
\hline $\begin{array}{c}\text { Gupta } \\
\text { et al.[57] }\end{array}$ & $\begin{array}{c}\text { Novel } \\
\text { approach }\end{array}$ & $\begin{array}{c}\text { LSTM } \\
\text { based } \\
\text { Deep } \\
\text { Learning } \\
\text { model }\end{array}$ & $\begin{array}{c}\text { Machine- } \\
\text { learning } \\
\text { (SVM, } \\
\text { Decision } \\
\text { Trees, Naive } \\
\text { Bayes) }\end{array}$ & $\begin{array}{c}\text { Angry, Happy, } \\
\text { Sad, Others }\end{array}$ & $\begin{array}{c}\text { Combines } \\
\text { sentiment } \\
\text { and semantic } \\
\text { based } \\
\text { embeddings; } \\
\text { Outperforms } \\
\text { most baseline } \\
\text { machine learning } \\
\text { approaches }\end{array}$ & $\begin{array}{l}\text { Unable to } \\
\text { handle } \\
\text { context }\end{array}$ \\
\hline $\begin{array}{l}\text { Desmet } \\
\text { et al.[58] }\end{array}$ & $\begin{array}{c}\text { Novel } \\
\text { approach }\end{array}$ & $\begin{array}{c}\text { NLP and } \\
\text { sentiment } \\
\text { mining }\end{array}$ & $\begin{array}{l}\text { Machine- } \\
\text { learning- } \\
\text { SVM }\end{array}$ & $\begin{array}{c}\text { Abuse, Anger, } \\
\text { Blame, Fear, } \\
\text { Forgiveness, } \\
\text { Guilt, } \\
\text { Happiness, } \\
\text { Hopefulness, } \\
\text { Hopelessness, } \\
\text { Information, } \\
\text { Instructions, } \\
\text { Love, } \\
\text { Pride, Sorrow, } \\
\text { Thankfulness }\end{array}$ & $\begin{array}{l}\text { Uses machine } \\
\text { learning for } \\
\text { emotion } \\
\text { detection in } \\
\text { suicide notes; } \\
\text { Uses both } \\
\text { semantic and } \\
\text { lexical } \\
\text { features like } \\
\text { bags-of-words, } \\
\text { POS tags } \\
\text { and trigrams }\end{array}$ & $\begin{array}{c}\text { Lack of } \\
\text { data; Unable } \\
\text { to recognize } \\
\text { rare } \\
\text { emotions; } \\
\text { Did not } \\
\text { consider } \\
\text { negations }\end{array}$ \\
\hline
\end{tabular}

Table 2.4: Related Works with Features and Limitations - Table III. 


\begin{tabular}{|c|c|c|c|c|c|c|}
\hline Authors & Type & Approach & Method & Emotions & Features & Limitations \\
\hline $\begin{array}{c}\text { Dini } \\
\text { et al. [59] }\end{array}$ & $\begin{array}{c}\text { Novel } \\
\text { approach }\end{array}$ & $\begin{array}{c}\text { Symbolic } \\
\text { and } \\
\text { Machine- } \\
\text { learning }\end{array}$ & $\begin{array}{l}\text { Lexical- } \\
\text { based } \\
\text { and } \\
\text { machine- } \\
\text { learning }\end{array}$ & $\begin{array}{c}\text { Anger, } \\
\text { Disgust, } \\
\text { Fear, Joy, } \\
\text { Sadness, } \\
\text { Surprise }\end{array}$ & $\begin{array}{c}\text { Creates Emotion } \\
\text { Tweet Corpus for } \\
\text { Classification } \\
\text { (ETCC) and } \\
\text { Emotion Tweet } \\
\text { Corpus for } \\
\text { Relevance (ETCR); } \\
\text { Uses word, } \\
\text { lemma, } \\
\text { noun phrase, } \\
\text { dependencies } \\
\text { between } \\
\text { POS as } \\
\text { feature set }\end{array}$ & $\begin{array}{c}\text { Quality } \\
\text { verification } \\
\text { needed for } \\
\text { two new } \\
\text { corpora }\end{array}$ \\
\hline $\begin{array}{c}\text { Mohammad } \\
\text { et al. }[60]\end{array}$ & $\begin{array}{c}\text { Novel } \\
\text { approach }\end{array}$ & $\begin{array}{c}\text { Best } \\
\text { Worst } \\
\text { Scaling } \\
\text { (BWS) }\end{array}$ & Regression & $\begin{array}{c}\text { Anger, } \\
\text { Fear, } \\
\text { Joy, } \\
\text { Sadness }\end{array}$ & $\begin{array}{l}\text { Detects emotion } \\
\text { intensity; Creates } \\
\text { four tweet } \\
\text { datasets; } \\
\text { Shows correlations } \\
\text { between emotion } \\
\text { pairs; Uses word } \\
\text { n-grams, character } \\
\text { n-grams, word } \\
\text { embeddings } \\
\text { and affect } \\
\text { lexicons } \\
\text { for regression }\end{array}$ & $\begin{array}{c}\text { Accuracy } \\
\text { not } \\
\text { verified }\end{array}$ \\
\hline $\begin{array}{l}\text { Summa } \\
\text { et al.[61] }\end{array}$ & $\begin{array}{c}\text { Novel } \\
\text { approach }\end{array}$ & $\begin{array}{l}\text { Interdis- } \\
\text { ciplinary } \\
\text { method }\end{array}$ & $\begin{array}{l}\text { Graph- } \\
\text { based } \\
\text { semi- } \\
\text { supervised } \\
\text { learning, } \\
\text { NLP }\end{array}$ & $\begin{array}{c}\text { Anger, } \\
\text { Happiness, } \\
\text { Disgust, } \\
\text { Fear, } \\
\text { Sadness, } \\
\text { Surprise, } \\
\text { None }\end{array}$ & $\begin{array}{c}\text { Combines } \\
\text { linguistic, } \\
\text { temporal, and } \\
\text { spatial } \\
\text { information; } \\
\text { Computes } \\
\text { similarity } \\
\text { between } \\
\text { tweet nodes }\end{array}$ & $\begin{array}{c}\text { Accuracy } \\
\text { was not } \\
\text { very high; } \\
\text { Parameters } \\
\text { selection } \\
\text { was random }\end{array}$ \\
\hline
\end{tabular}

Table 2.5: Related Works with Features and Limitations - Table IV. 


\begin{tabular}{|c|c|c|c|c|c|c|}
\hline Authors & Type & Approach & Method & Emotions & Features & Limitations \\
\hline $\begin{array}{c}\text { Sen } \\
\text { et } \\
\text { al. }[62]\end{array}$ & $\begin{array}{c}\text { Novel } \\
\text { approach }\end{array}$ & $\begin{array}{l}\text { Jointly } \\
\text { learnt } \\
\text { model for } \\
\text { emotion } \\
\text { and } \\
\text { sentiment }\end{array}$ & $\begin{array}{c}\text { Machine- } \\
\text { learning } \\
\text { (SVM, } \\
\text { CNN) }\end{array}$ & $\begin{array}{c}\text { Emotion - } \\
\text { Anger, } \\
\text { Amusement, } \\
\text { Excitement, } \\
\text { Happiness, } \\
\text { Hope, Love, } \\
\text { Sadness; } \\
\text { Sentiment - } \\
\text { Positive, } \\
\text { Negative, } \\
\text { Neutral, } \\
\text { None }\end{array}$ & $\begin{array}{l}\text { Uses sentiments as } \\
\text { auxiliary input } \\
\text { for textual emotion } \\
\text { detection; Uses } \\
\text { multi-tasking NN } \\
\text { for embedding } \\
\text { learning }\end{array}$ & - \\
\hline $\begin{array}{c}\text { Jain } \\
\text { et } \\
\text { al.[63] }\end{array}$ & $\begin{array}{c}\text { Survey } \\
\text { and a } \\
\text { proposed } \\
\text { approach }\end{array}$ & $\begin{array}{c}\text { Machine- } \\
\text { learning } \\
\text { (SVM, } \\
\text { Naive } \\
\text { Bayes) }\end{array}$ & $\begin{array}{l}\text { Intelligent } \\
\text { text } \\
\text { processing }\end{array}$ & $\begin{array}{c}\text { Basic } \\
\text { Emotion } \\
\text { Theories, } \\
\text { Ekman } \\
\text { (for } \\
\text { framework) }\end{array}$ & $\begin{array}{c}\text { Compilation of } \\
\text { different emotion } \\
\text { detection methods } \\
\text { in existing } \\
\text { researches; Detail } \\
\text { survey on election } \\
\text { prediction tweets; } \\
\text { Combines } \\
\text { corpus-based } \\
\text { features and } \\
\text { emotion related } \\
\text { features for } \\
\text { multilingual text } \\
\text { emotion detection }\end{array}$ & $\begin{array}{c}\text { Proposed } \\
\text { framework } \\
\text { focuses on } \\
\text { only } \\
\text { political, } \\
\text { health and } \\
\text { sports } \\
\text { domain }\end{array}$ \\
\hline $\begin{array}{c}\text { Kang } \\
\text { et. } \\
\text { al.[64] }\end{array}$ & $\begin{array}{c}\text { Novel } \\
\text { approach }\end{array}$ & $\begin{array}{c}\text { Bayesian } \\
\text { Model }\end{array}$ & $\begin{array}{l}\text { Bayesian } \\
\text { Inference } \\
\text { Method }\end{array}$ & $\begin{array}{c}\text { Anger, } \\
\text { Anxiety, } \\
\text { Expect, Hate, } \\
\text { Joy, Love, } \\
\text { Sorrow, } \\
\text { Surprise }\end{array}$ & $\begin{array}{c}\text { Works with } \\
\text { contextual } \\
\text { information } \\
\text { to get the latent } \\
\text { semantic } \\
\text { dimensions; } \\
\text { Predicts emotion } \\
\text { for both word and } \\
\text { document text; } \\
\text { Two Bayesian } \\
\text { models DWET } \\
\text { and HDWET were } \\
\text { applied; DWET } \\
\text { outperforms all } \\
\text { baseline methods. }\end{array}$ & $\begin{array}{c}\text { Bayesian } \\
\text { model } \\
\text { will never } \\
\text { converge } \\
\text { if the } \\
\text { semantic } \\
\text { dimensions } \\
\text { increase } \\
\text { much; } \\
\text { Works with } \\
\text { Chinese } \\
\text { language } \\
\text { only }\end{array}$ \\
\hline
\end{tabular}

Table 2.6: Related Works with Features and Limitations - Table V. 


\begin{tabular}{|c|c|c|c|c|c|c|}
\hline Authors & Type & Approach & Method & Emotions & Features & Limitations \\
\hline $\begin{array}{c}\text { Perikos } \\
\text { et. } \\
\text { al.[13] }\end{array}$ & $\begin{array}{l}\text { Ensemble } \\
\text { Approach }\end{array}$ & $\begin{array}{l}\text { Machine } \\
\text { Learning }\end{array}$ & $\begin{array}{c}\text { Ensemble } \\
\text { of } \\
\text { Naive } \\
\text { Bayes, } \\
\text { Maximum } \\
\text { Entropy } \\
\text { learner, } \\
\text { Knowledge- } \\
\text { based } \\
\text { Tool }\end{array}$ & $\begin{array}{l}\text { Circum- } \\
\text { plex } \\
\text { Model }\end{array}$ & $\begin{array}{c}\text { A sentence-level } \\
\text { emotion and } \\
\text { polarity } \\
\text { detector; } \\
\text { Analyzes } \\
\text { each sentence for } \\
\text { feature extraction } \\
\text { and inserts them } \\
\text { into three } \\
\text { classifiers; } \\
\text { Output is } \\
\text { decided } \\
\text { by a voting } \\
\text { system }\end{array}$ & $\begin{array}{l}\text { Lack of } \\
\text { lexical } \\
\text { resources; } \\
\text { Accuracy } \\
\text { is not } \\
\text { better } \\
\text { than all } \\
\text { baseline } \\
\text { methods }\end{array}$ \\
\hline $\begin{array}{l}\text { Nahin } \\
\text { et. } \\
\text { al. [14] }\end{array}$ & $\begin{array}{c}\text { Novel } \\
\text { approach }\end{array}$ & $\begin{array}{c}\text { Machine } \\
\text { Learning } \\
\text { (SVM } \\
\text { with } \\
\text { Jaccard } \\
\text { Similarity) }\end{array}$ & $\begin{array}{l}\text { Combin- } \\
\text { ation } \\
\text { of } \\
\text { keystroke } \\
\text { and text } \\
\text { pattern } \\
\text { analysis }\end{array}$ & $\begin{array}{c}\text { Anger, } \\
\text { Disgust, } \\
\text { Fear, } \\
\text { Guilt, } \\
\text { Joy, } \\
\text { Sadness, } \\
\text { Shame }\end{array}$ & $\begin{array}{l}\text { Collects user } \\
\text { keystrokes and } \\
\text { emotions with } \\
\text { and without some } \\
\text { specific texts; } \\
\text { Uses this } \\
\text { data to train } \\
\text { and test the } \\
\text { classifiers }\end{array}$ & $\begin{array}{c}\text { Lack of } \\
\text { proper } \\
\text { user } \\
\text { participation; } \\
\text { Time } \\
\text { consuming } \\
\text { data } \\
\text { collection; } \\
\text { Lack of } \\
\text { adequate } \\
\text { words in } \\
\text { dataset }\end{array}$ \\
\hline $\begin{array}{l}\text { Moha- } \\
\text { mmad } \\
\text { et } \\
\text { al.[15] }\end{array}$ & $\begin{array}{c}\text { Novel } \\
\text { approach }\end{array}$ & $\begin{array}{l}\text { Machine } \\
\text { Learning } \\
\text { (Majority } \\
\text { classifier, } \\
\text { SVM) }\end{array}$ & $\begin{array}{c}\text { Machine } \\
\text { Learning } \\
\text { with } \\
\text { combin- } \\
\text { ations } \\
\text { of } 11 \\
\text { feature } \\
\text { sets } \\
\text { containing } \\
\text { different } \\
\text { features }\end{array}$ & $\begin{array}{c}\text { Anger, } \\
\text { Disgust, } \\
\text { Fear, } \\
\text { Joy, } \\
\text { Sadness, } \\
\text { Surprise }\end{array}$ & $\begin{array}{c}\text { Collection of } \\
\text { emotion tweets } \\
\text { labeled with } \\
\text { hashtag emotion } \\
\text { words; Generation } \\
\text { of a large } \\
\text { word-emotion } \\
\text { association lexicon; } \\
\text { Detection of both } \\
\text { emotion and } \\
\text { personality; } \\
\text { Calculation of } \\
\text { extroversion, } \\
\text { neuroticism, } \\
\text { agreeably, } \\
\text { conscientiousness } \\
\text { and openness }\end{array}$ & - \\
\hline
\end{tabular}

Table 2.7: Related Works with Features and Limitations - Table VI. 


\begin{tabular}{|c|c|c|c|c|c|c|}
\hline Authors & Type & Approach & Method & Emotions & Features & Limitations \\
\hline $\begin{array}{c}\text { Agrawal } \\
\text { et. } \\
\text { al. }[65]\end{array}$ & $\begin{array}{c}\text { Novel } \\
\text { approach }\end{array}$ & $\begin{array}{l}\text { Machine } \\
\text { Learning }\end{array}$ & $\begin{array}{l}\text { Unsuper- } \\
\text { vised } \\
\text { Context- } \\
\text { based } \\
\text { Method }\end{array}$ & $\begin{array}{c}\text { Ekman's } \\
\text { Model }\end{array}$ & $\begin{array}{l}\text { Unsupervised approach } \\
\text { classifies emotions } \\
\text { based on the } \\
\text { semantic } \\
\text { relationships } \\
\text { of Noun, Verb, } \\
\text { Adjective and } \\
\text { Adverb; Generates } \\
\text { emotion vectors } \\
\text { with NAVA; } \\
\text { Uses a few emotion } \\
\text { representative words } \\
\text { to start; Does not } \\
\text { need any annotated } \\
\text { dataset or lexicon; } \\
\text { Accurate than other } \\
\text { unsupervised and } \\
\text { some supervised } \\
\text { method; } \\
\text { Can work with any } \\
\text { emotion model }\end{array}$ & $\begin{array}{l}\text { Semantic } \\
\text { relatedness } \\
\text { is } \\
\text { dependent } \\
\text { on the } \\
\text { corpus }\end{array}$ \\
\hline $\begin{array}{c}\text { Tiwari } \\
\text { et. } \\
\text { al.[66] }\end{array}$ & $\begin{array}{c}\text { Novel } \\
\text { Approach }\end{array}$ & $\begin{array}{c}\text { Hybrid } \\
\text { Approach }\end{array}$ & $\begin{array}{l}\text { Hybrid of } \\
\text { Machine } \\
\text { Learning } \\
\text { and Rule } \\
\text { based } \\
\text { methods }\end{array}$ & $\begin{array}{c}\text { Ekman's } \\
\text { Model }\end{array}$ & $\begin{array}{c}\text { Detail explanations } \\
\text { about emotion, } \\
\text { emotion models, } \\
\text { emotion detection } \\
\text { methods and their } \\
\text { limitations; Uses } \\
\text { a combination } \\
\text { of machine learning } \\
\text { and rule based } \\
\text { method; Uses emotion } \\
\text { vectors with } \\
\text { NAVA extracted by } \\
\text { POS tagger }\end{array}$ & $\begin{array}{l}\text { Uses a } \\
\text { small } \\
\text { dataset } \\
\text { which } \\
\text { effects the } \\
\text { general- } \\
\text { ization } \\
\text { and } \\
\text { standards } \\
\text { of the } \\
\text { results }\end{array}$ \\
\hline $\begin{array}{c}\text { Lee } \\
\text { et. } \\
\text { al. }[67]\end{array}$ & $\begin{array}{c}\text { Novel } \\
\text { Approach }\end{array}$ & $\begin{array}{l}\text { Emotion- } \\
\text { Cause } \\
\text { Detection }\end{array}$ & $\begin{array}{l}\text { Rule- } \\
\text { based } \\
\text { emotion- } \\
\text { cause } \\
\text { detection }\end{array}$ & $\begin{array}{c}\text { Anger, } \\
\text { Fear, } \\
\text { Happiness, } \\
\text { Sadness, } \\
\text { Surprise }\end{array}$ & $\begin{array}{l}\text { Generated a Chinese } \\
\text { annotated emotion- } \\
\text { cause corpus; Linguistic } \\
\text { analysis was done } \\
\text { to find out the } \\
\text { correlation between } \\
\text { emotion and cause; } \\
\text { Linguistic rules used } \\
\text { for cause detection }\end{array}$ & $\begin{array}{c}\text { Works } \\
\text { with } \\
\text { Chinese } \\
\text { language } \\
\text { only }\end{array}$ \\
\hline
\end{tabular}

Table 2.8: Related Works with Features and Limitations - Table VII. 


\begin{tabular}{|c|c|c|c|c|c|c|}
\hline Authors & Type & Approach & Method & Emotions & Features & Limitations \\
\hline $\begin{array}{c}\mathrm{Li} \\
\text { et. } \\
\text { al.[68] }\end{array}$ & $\begin{array}{c}\text { Novel } \\
\text { Approach }\end{array}$ & $\begin{array}{c}\text { Emotion } \\
\text { and } \\
\text { Cause } \\
\text { Detection } \\
\text { and } \\
\text { analysis }\end{array}$ & $\begin{array}{c}\text { Rule- } \\
\text { based } \\
\text { emotion- } \\
\text { cause } \\
\text { detection } \\
\text { and } \\
\text { machine } \\
\text { learning } \\
\text { based } \\
\text { emotion } \\
\text { detection }\end{array}$ & $\begin{array}{c}\text { Ekman's } \\
\text { Model }\end{array}$ & $\begin{array}{c}\text { Works with } \\
\text { Chinese } \\
\text { micro-blog data; } \\
\text { Emotion-cause } \\
\text { extraction } \\
\text { system was } \\
\text { used to } \\
\text { extract the } \\
\text { features; } \\
\text { System was } \\
\text { trained } \\
\text { with that data; } \\
\text { SVM was } \\
\text { used to } \\
\text { detect emotion }\end{array}$ & $\begin{array}{c}\text { Works } \\
\text { with } \\
\text { Chinese } \\
\text { language } \\
\text { only; Do } \\
\text { not work } \\
\text { well with } \\
\text { complicated } \\
\text { linguistic } \\
\text { patterns }\end{array}$ \\
\hline $\begin{array}{c}\text { An } \\
\text { et. } \\
\text { al. }[69]\end{array}$ & $\begin{array}{c}\text { Novel } \\
\text { Approach }\end{array}$ & $\begin{array}{c}\text { Machine } \\
\text { Learning } \\
\text { based } \\
\text { approach } \\
\text { for emotion } \\
\text { detection } \\
\text { in song } \\
\text { lyrics }\end{array}$ & $\begin{array}{c}\text { Machine } \\
\text { Learning } \\
\text { (Naive } \\
\text { Bayes) }\end{array}$ & $\begin{array}{c}\text { Comfortable, } \\
\text { Happy, } \\
\text { Inspirational, } \\
\text { Joyful, } \\
\text { Lonely, } \\
\text { Miss, } \\
\text { Nostalgic, } \\
\text { Passionate, } \\
\text { Quiet, } \\
\text { Relaxed, } \\
\text { Romantic, } \\
\text { Sad, } \\
\text { Soulful, } \\
\text { Sweet, } \\
\text { Yearning }\end{array}$ & $\begin{array}{c}\text { Naive Bayes } \\
\text { classifier } \\
\text { was used for } \\
\text { emotion } \\
\text { classifications from } \\
\text { music lyrics; } \\
\text { Positivity and } \\
\text { negativity } \\
\text { of emotions were } \\
\text { also analyzed; Four } \\
\text { different dataset of } \\
\text { English and } \\
\text { Chinese } \\
\text { music lyrics } \\
\text { were used } \\
\text { for classification }\end{array}$ & $\begin{array}{c}\text { The } \\
\text { result } \\
\text { for } \\
\text { English } \\
\text { lyrics } \\
\text { were } \\
\text { not as } \\
\text { accurate } \\
\text { as } \\
\text { Chinese } \\
\text { lyrics }\end{array}$ \\
\hline $\begin{array}{l}\text { Hajar } \\
\text { et. } \\
\text { al.[70] }\end{array}$ & $\begin{array}{c}\text { Novel } \\
\text { Approach }\end{array}$ & $\begin{array}{l}\text { Machine } \\
\text { Learning }\end{array}$ & $\begin{array}{c}\text { Unsuper- } \\
\text { vised } \\
\text { Machine } \\
\text { Learning } \\
\text { Approach }\end{array}$ & $\begin{array}{c}\text { Ekman's } \\
\text { Model }\end{array}$ & $\begin{array}{c}\text { You tube } \\
\text { comments } \\
\text { texts were used for } \\
\text { emotion detection; } \\
\text { NAVA words were } \\
\text { extracted from } \\
\text { the sentences; } \\
\text { PMI for each } \\
\text { emotion word } \\
\text { against } \\
\text { NAVA words were } \\
\text { calculated for } \\
\text { measuring the } \\
\text { correlations }\end{array}$ & - \\
\hline
\end{tabular}

Table 2.9: Related Works with Features and Limitations - Table VIII. 


\begin{tabular}{|c|c|c|c|c|c|c|}
\hline Authors & Type & Approach & Method & Emotions & Features & Limitations \\
\hline $\begin{array}{c}\mathrm{Li} \\
\text { et. } \\
\text { al. }[71]\end{array}$ & $\begin{array}{c}\text { Novel } \\
\text { Approach }\end{array}$ & $\begin{array}{l}\text { Machine } \\
\text { Learning }\end{array}$ & $\begin{array}{c}\text { Hybrid of } \\
\text { Neural } \\
\text { Networks } \\
\text { (Biterm } \\
\text { Topic } \\
\text { Model and } \\
\text { Convolution } \\
\text { Neural } \\
\text { Network) }\end{array}$ & $\begin{array}{c}\text { Ekman's } \\
\text { Model }\end{array}$ & $\begin{array}{l}\text { Uses Biterm Topic } \\
\text { Model(BTM) } \\
\text { which uses } \\
\text { probabilistic latent } \\
\text { semantic analysis } \\
\text { on the words of a } \\
\text { text to find out } \\
\text { the co-occurrence } \\
\text { patterns; BTM is } \\
\text { used to learn } \\
\text { the layers of a } \\
\text { neural network; } \\
\text { Back-propagation } \\
\text { method is used to } \\
\text { fine-tune the } \\
\text { network }\end{array}$ & $\begin{array}{c}\text { User } \\
\text { context } \\
\text { was not } \\
\text { considered }\end{array}$ \\
\hline $\begin{array}{c}\text { Strapp- } \\
\text { arava } \\
\text { et. } \\
\text { al.[72] }\end{array}$ & $\begin{array}{c}\text { Compar- } \\
\text { ative } \\
\text { Study }\end{array}$ & $\begin{array}{l}\text { Existing } \\
\text { Methods }\end{array}$ & $\begin{array}{c}\text { Keyword- } \\
\text { based, } \\
\text { Lexicon- } \\
\text { based, } \\
\text { Supervised } \\
\text { and } \\
\text { Unsupervised } \\
\text { Machine } \\
\text { Learning } \\
\text { based } \\
\text { Methods }\end{array}$ & $\begin{array}{c}\text { Ekman's } \\
\text { Model }\end{array}$ & $\begin{array}{l}\text { A comparative } \\
\text { study to find } \\
\text { out which } \\
\text { method is } \\
\text { better for } \\
\text { annotating } \\
\text { datasets }\end{array}$ & $\begin{array}{c}\text { No decision } \\
\text { was made } \\
\text { after the } \\
\text { comparisons; } \\
\text { Not a } \\
\text { complete } \\
\text { survey on } \\
\text { emotion } \\
\text { detection; } \\
\text { No new } \\
\text { approach } \\
\text { was } \\
\text { proposed }\end{array}$ \\
\hline $\begin{array}{l}\text { Ghazi } \\
\text { et. } \\
\text { al. [73] }\end{array}$ & $\begin{array}{c}\text { Novel } \\
\text { Approach }\end{array}$ & $\begin{array}{l}\text { Hybrid } \\
\text { Method }\end{array}$ & $\begin{array}{l}\text { Hybrid of } \\
\text { keyword- } \\
\text { based, } \\
\text { lexicon-based } \\
\text { and machine } \\
\text { learning } \\
\text { based } \\
\text { approaches } \\
\text { with } \\
\text { logical } \\
\text { regression }\end{array}$ & $\begin{array}{c}\text { Ekman's } \\
\text { Model }\end{array}$ & $\begin{array}{l}\text { Uses keywords to } \\
\text { find to match with } \\
\text { the sentences; } \\
\text { Extracts syntactic } \\
\text { and semantic } \\
\text { features from } \\
\text { the sentences; } \\
\text { Applies machine } \\
\text { learning to classify } \\
\text { the sentences } \\
\text { according to } \\
\text { their emotions } \\
\text { by using the } \\
\text { extracted features }\end{array}$ & $\begin{array}{c}\text { Lack of } \\
\text { generalization } \\
\text { of features; } \\
\text { Indirect } \\
\text { dependencies } \\
\text { between } \\
\text { emotion word } \\
\text { and other } \\
\text { words of the } \\
\text { sentence was } \\
\text { ignored }\end{array}$ \\
\hline
\end{tabular}

Table 2.10: Related Works with Features and Limitations - Table IX. 


\begin{tabular}{|c|c|c|c|c|c|c|}
\hline Authors & Type & Approach & Method & Emotions & Features & Limitations \\
\hline $\begin{array}{c}\text { Hasan } \\
\text { et. } \\
\text { al. [74] }\end{array}$ & $\begin{array}{c}\text { Novel } \\
\text { Approach }\end{array}$ & $\begin{array}{l}\text { Machine } \\
\text { Learning }\end{array}$ & $\begin{array}{c}\text { Naive } \\
\text { Bayes, } \\
\text { Support } \\
\text { Vector } \\
\text { Machine, } \\
\text { Decision } \\
\text { Tree, } \\
\text { K Nearest } \\
\text { Neighbor }\end{array}$ & $\begin{array}{l}\text { Circum- } \\
\text { plex } \\
\text { Model }\end{array}$ & $\begin{array}{c}\text { Uses hashtag } \\
\text { words, } \\
\text { punctuations, } \\
\text { emoticons and } \\
\text { negations } \\
\text { as features; Uses } \\
\text { hashtags to label } \\
\text { the tweets } \\
\text { automatically }\end{array}$ & - \\
\hline $\begin{array}{c}\text { Hasan } \\
\text { et. } \\
\text { al.[75] }\end{array}$ & $\begin{array}{c}\text { Novel } \\
\text { Approach }\end{array}$ & $\begin{array}{l}\text { Machine } \\
\text { Learning }\end{array}$ & $\begin{array}{c}\text { Emotex } \\
\text { Classifier }\end{array}$ & $\begin{array}{c}\text { Angry, } \\
\text { Happy, } \\
\text { Sad }\end{array}$ & $\begin{array}{c}\text { Filters } \\
\text { tweets with } \\
\text { a binary } \\
\text { classifier } \\
\text { to find out the } \\
\text { tweets with } \\
\text { emotion; } \\
\text { Applies Emotex } \\
\text { to extract } \\
\text { features; } \\
\text { Classifies tweets } \\
\text { about a public } \\
\text { event; Generates } \\
\text { decision about } \\
\text { public mood } \\
\text { on an } \\
\text { event; Tracks } \\
\text { temporal changes } \\
\text { in public emotions }\end{array}$ & $\begin{array}{c}\text { Considers } \\
\text { only three } \\
\text { emotions }\end{array}$ \\
\hline $\begin{array}{c}\text { Kanger } \\
\text { et. } \\
\text { al. }[76]\end{array}$ & $\begin{array}{c}\text { Novel } \\
\text { Approach }\end{array}$ & $\begin{array}{l}\text { Machine } \\
\text { Learning }\end{array}$ & $\begin{array}{c}\text { Combination } \\
\text { of fuzzy } \\
\text { logic and } \\
\text { Neural } \\
\text { Network }\end{array}$ & $\begin{array}{c}\text { Anger, } \\
\text { Happy, } \\
\text { Sad }\end{array}$ & $\begin{array}{l}\text { Applies hybrid of } \\
\text { fuzzy logic and } \\
\text { neural network; } \\
\text { Creates fuzzy } \\
\text { rules and extracts } \\
\text { features from the } \\
\text { input data }\end{array}$ & $\begin{array}{l}\text { Absence of } \\
\text { results on } \\
\text { benchmark } \\
\text { data; } \\
\text { Lack of } \\
\text { generalization } \\
\text { for any } \\
\text { domain; Lack } \\
\text { of detail } \\
\text { of some } \\
\text { steps of the } \\
\text { approach; } \\
\text { Works } \\
\text { with three } \\
\text { emotions } \\
\text { only }\end{array}$ \\
\hline
\end{tabular}

Table 2.11: Related Works with Features and Limitations - Table X. 


\begin{tabular}{|c|c|c|c|c|c|c|}
\hline Authors & Type & Approach & Method & Emotions & Features & Limitations \\
\hline $\begin{array}{c}\text { Grover } \\
\text { et. } \\
\text { al. }[77]\end{array}$ & $\begin{array}{c}\text { Hybrid } \\
\text { Approach }\end{array}$ & $\begin{array}{c}\text { Hybrid of } \\
\text { Keyword- } \\
\text { based and } \\
\text { Machine } \\
\text { Learning }\end{array}$ & $\begin{array}{c}\text { Keyword- } \\
\text { based and } \\
\text { Machine- } \\
\text { Learning } \\
\text { (Naive } \\
\text { Bayes } \\
\text { and SVM) }\end{array}$ & $\begin{array}{c}\text { Ekman's } \\
\text { Model }\end{array}$ & $\begin{array}{l}\text { Tokenizes the data; } \\
\text { After removing } \\
\text { stop words applies } \\
\text { stemming steps; } \\
\text { Extracts features } \\
\text { and applies rule-based } \\
\text { engine to filter data } \\
\text { with emotions; } \\
\text { Uses classifiers } \\
\text { to classify the } \\
\text { filtered data }\end{array}$ & $\begin{array}{c}\text { Works } \\
\text { with } \\
\text { Punjabi } \\
\text { language } \\
\text { only }\end{array}$ \\
\hline $\begin{array}{c}\text { Joshi } \\
\text { et. } \\
\text { al. }[78]\end{array}$ & $\begin{array}{c}\text { Novel } \\
\text { Approach }\end{array}$ & $\begin{array}{l}\text { Lexicon- } \\
\text { based } \\
\text { Approach }\end{array}$ & $\begin{array}{c}\text { Lexicon } \\
\text { (LIWC } \\
\text { and } \\
\text { Emo- } \\
\text { Lex) } \\
\text { based } \\
\text { Emotion } \\
\text { Tracker }\end{array}$ & $\begin{array}{c}\text { Angry, } \\
\text { Anxious, } \\
\text { Happy, } \\
\text { Sad }\end{array}$ & $\begin{array}{c}\text { Develops an emotion } \\
\text { tracker; Downloads } \\
\text { tweets, } \\
\text { predicts emotion, } \\
\text { predicts overall } \\
\text { emotion in a time } \\
\text {-period; Generates } \\
\text { and shows emotion } \\
\text { time-sequence graphs; } \\
\text { Computes the } \\
\text { excitement } \\
\text { levels of a } \\
\text { cricket match, } \\
\text { emotions } \\
\text { between characters } \\
\text { of a play and } \\
\text { emotions towards a } \\
\text { product accurately }\end{array}$ & $\begin{array}{c}\text { Lack of } \\
\text { detection } \\
\text { of } \\
\text { subjectivity } \\
\text { and emotion } \\
\text { interactions }\end{array}$ \\
\hline
\end{tabular}

Table 2.12: Related Works with Features and Limitations - Table XI. 


\begin{tabular}{|c|c|c|c|c|c|c|}
\hline Features & $\begin{array}{c}\text { Binali } \\
\text { et al. } \\
{[54]}\end{array}$ & $\begin{array}{l}\text { Kao } \\
\text { et al. } \\
{[55]}\end{array}$ & $\begin{array}{c}\text { Shivhare } \\
\text { et al. } \\
{[56]}\end{array}$ & $\begin{array}{c}\text { Gupta } \\
\text { et al. } \\
{[57]}\end{array}$ & $\begin{array}{l}\text { Desmet } \\
\text { et al. } \\
{[58]}\end{array}$ & $\begin{array}{l}\text { Dini } \\
\text { et al. } \\
{[59]}\end{array}$ \\
\hline Keyword-based & $\mathrm{X}$ & & & & & \\
\hline Lexicon-based & & & & & & $\mathrm{X}$ \\
\hline Learning-based & $\mathrm{X}$ & & & $\mathrm{X}$ & $\mathrm{X}$ & $\mathrm{X}$ \\
\hline Supervised Machine Learning-based & & & & $\mathrm{X}$ & $\mathrm{X}$ & \\
\hline Support Vector Machine & & & & & $\mathrm{X}$ & \\
\hline Naive Bayes & & & & $\mathrm{X}$ & & \\
\hline Bayesian Models & & & & & & \\
\hline Decision Tree & & & & $\mathrm{X}$ & & \\
\hline K-Nearest Neighbor & & & & & & \\
\hline Neural Networks & & & & & & \\
\hline Maximum Entropy & & & & & & \\
\hline Majority Classifier & & & & & & \\
\hline Unsupervised Machine Learning-based & & & & & & \\
\hline Semi-supervised Machine Learning-based & & & & & & \\
\hline Hybrid Method & $\mathrm{X}$ & & & & & \\
\hline Ensemble Method & & & & & & \\
\hline Fuzzy-Logic & & & & & & \\
\hline Rule-based Method & & & & & & \\
\hline Reasoning Method & & $\mathrm{X}$ & & & & \\
\hline Semantic-based Method & & $\mathrm{X}$ & & $\mathrm{X}$ & $\mathrm{X}$ & \\
\hline Ontology-based Method & & & $\mathrm{X}$ & & & \\
\hline Regression-based Method & & & & & & \\
\hline Uses Sentiment & & & $\mathrm{X}$ & $\mathrm{X}$ & & \\
\hline Generation of new Data Corpus & & & & & & $\mathrm{X}$ \\
\hline Generation of new Lexicon & & & & & & \\
\hline Works with English Language & $\mathrm{X}$ & $\mathrm{X}$ & $\mathrm{X}$ & $\mathrm{X}$ & $\mathrm{X}$ & $\mathrm{X}$ \\
\hline Works with Other Languages & & & & & & \\
\hline Detects Single Emotion & $\mathrm{X}$ & $\mathrm{X}$ & $\mathrm{X}$ & $\mathrm{X}$ & $\mathrm{X}$ & $\mathrm{X}$ \\
\hline Detects Multiple Emotions & & & & & & \\
\hline Word-level Detection & $\mathrm{X}$ & & $\mathrm{X}$ & $\mathrm{X}$ & $\mathrm{X}$ & $\mathrm{X}$ \\
\hline Sentence-level Detection & & $\mathrm{X}$ & & & & \\
\hline Document-level Detection & & & & & & \\
\hline Emotion Intensity Detection & & & & & & \\
\hline Personality Detection & & & & & & \\
\hline Emotion-Cause Detection & & & & & & \\
\hline Public Mood Detection & & & & & & \\
\hline Temporal Effect & & & & & & \\
\hline
\end{tabular}

Table 2.13: Features used in Existing Works - Table I. 


\begin{tabular}{|c|c|c|c|c|c|c|}
\hline Features & $\begin{array}{c}\text { Kang } \\
\text { et. al. } \\
{[64]}\end{array}$ & $\begin{array}{c}\text { Perikos } \\
\text { et. al. } \\
{[13]}\end{array}$ & $\begin{array}{c}\text { Nahin } \\
\text { et. al. } \\
{[14]}\end{array}$ & $\begin{array}{c}\text { Moha- } \\
\text { mmad } \\
\text { et al. } \\
{[15]}\end{array}$ & $\begin{array}{c}\text { Agrawal } \\
\text { et. al. } \\
{[65]}\end{array}$ & $\begin{array}{c}\text { Tiwari } \\
\text { et. al. } \\
{[66]}\end{array}$ \\
\hline \multicolumn{7}{|l|}{ Keyword-based } \\
\hline \multicolumn{7}{|l|}{ Lexicon-based } \\
\hline Learning-based & $\mathrm{X}$ & $\mathrm{X}$ & $\mathrm{X}$ & $\mathrm{X}$ & $\mathrm{X}$ & $\mathrm{X}$ \\
\hline Supervised Machine Learning-based & $\mathrm{X}$ & $\mathrm{X}$ & $\mathrm{X}$ & $\mathrm{X}$ & & \\
\hline Support Vector Machine & & & & $\mathrm{X}$ & & \\
\hline Naive Bayes & & $\mathrm{X}$ & & & & \\
\hline Bayesian Models & $\mathrm{X}$ & & & & & \\
\hline \multicolumn{7}{|l|}{ Decision Tree } \\
\hline \multicolumn{7}{|l|}{ K-Nearest Neighbor } \\
\hline \multicolumn{7}{|l|}{ Neural Networks } \\
\hline Maximum Entropy & & $\mathrm{X}$ & & & & \\
\hline Majority Classifier & & & & $\mathrm{X}$ & & \\
\hline Unsupervised Machine Learning-based & & & & & $\mathrm{X}$ & \\
\hline \multicolumn{7}{|l|}{ Semi-supervised Machine Learning-based } \\
\hline Hybrid Method & & & & & & $\mathrm{X}$ \\
\hline Ensemble Method & & $\mathrm{X}$ & & & & \\
\hline \multicolumn{7}{|l|}{ Fuzzy-Logic } \\
\hline Rule-based Method & & & & & & $\mathrm{X}$ \\
\hline \multicolumn{7}{|l|}{ Reasoning Method } \\
\hline Semantic-based Method & $\mathrm{X}$ & & & & $\mathrm{X}$ & \\
\hline \multicolumn{7}{|l|}{ Ontology-based Method } \\
\hline \multicolumn{7}{|l|}{ Regression-based Method } \\
\hline Uses Sentiment & & $\mathrm{X}$ & & & & \\
\hline \multicolumn{7}{|l|}{ Generation of new Data Corpus } \\
\hline Generation of new Lexicon & & & & $\mathrm{X}$ & & \\
\hline Works with English Language & & & $\mathrm{X}$ & $\mathrm{X}$ & $\mathrm{X}$ & $\mathrm{X}$ \\
\hline Works with Other Languages & $\mathrm{X}$ & $\mathrm{X}$ & & & & \\
\hline Detects Single Emotion & $\mathrm{X}$ & $\mathrm{X}$ & $\mathrm{X}$ & $\mathrm{X}$ & $\mathrm{X}$ & $\mathrm{X}$ \\
\hline \multicolumn{7}{|l|}{ Detects Multiple Emotions } \\
\hline Word-level Detection & $\mathrm{X}$ & & $\mathrm{X}$ & $\mathrm{X}$ & $\mathrm{X}$ & $\mathrm{X}$ \\
\hline Sentence-level Detection & & $\mathrm{X}$ & & & & \\
\hline Document-level Detection & $\mathrm{X}$ & & & & & \\
\hline \multicolumn{7}{|l|}{ Emotion Intensity Detection } \\
\hline Personality Detection & & & & $\mathrm{X}$ & & \\
\hline \multicolumn{7}{|l|}{ Emotion-Cause Detection } \\
\hline \multicolumn{7}{|l|}{ Public Mood Detection } \\
\hline Temporal Effect & & & & & & \\
\hline
\end{tabular}

Table 2.14: Features used in Existing Works - Table II. 


\begin{tabular}{|c|c|c|c|c|c|c|}
\hline Features & $\begin{array}{c}\text { Hajar } \\
\text { et. al. } \\
{[70]}\end{array}$ & $\begin{array}{c}\text { Li } \\
\text { et. al. } \\
{[71]}\end{array}$ & $\begin{array}{c}\text { Strapparava } \\
\text { et. al. } \\
{[72]}\end{array}$ & $\begin{array}{c}\text { Ghazi } \\
\text { et. al. } \\
{[73]}\end{array}$ & $\begin{array}{c}\text { Hasan } \\
\text { et. al. } \\
{[74]}\end{array}$ & $\begin{array}{c}\text { Hasan } \\
\text { et. al. } \\
{[75]}\end{array}$ \\
\hline \hline Keyword-based & & & $\mathrm{X}$ & $\mathrm{X}$ & & \\
\hline Lexicon-based & & & $\mathrm{X}$ & $\mathrm{X}$ & $\mathrm{X}$ & $\mathrm{X}$ \\
\hline Learning-based & $\mathrm{X}$ & $\mathrm{X}$ & $\mathrm{X}$ & $\mathrm{X}$ & $\mathrm{X}$ & $\mathrm{X}$ \\
\hline Supervised Machine Learning-based & & $\mathrm{X}$ & $\mathrm{X}$ & $\mathrm{X}$ & $\mathrm{X}$ & $\mathrm{X}$ \\
\hline Support Vector Machine & & & & $\mathrm{X}$ & $\mathrm{X}$ & $\mathrm{X}$ \\
\hline Naive Bayes & & & $\mathrm{X}$ & & $\mathrm{X}$ & $\mathrm{X}$ \\
\hline Bayesian Models & & & & & & \\
\hline Decision Tree & & & & & $\mathrm{X}$ & $\mathrm{X}$ \\
\hline K-Nearest Neighbor & & $\mathrm{X}$ & & & & $\mathrm{X}$ \\
\hline Neural Networks & & & & & & \\
\hline Maximum Entropy & & & & & & \\
\hline Majority Classifier & $\mathrm{X}$ & & & & & \\
\hline Unsupervised Machine Learning-based & & & & & & \\
\hline Semi-supervised Machine Learning-based & & $\mathrm{X}$ & & $\mathrm{X}$ & & \\
\hline Hybrid Method & & & & & & \\
\hline Ensemble Method & & & & & & \\
\hline Fuzzy-Logic & & & & & & \\
\hline Rule-based Method & & & & & & \\
\hline Reasoning Method & & $\mathrm{X}$ & & $\mathrm{X}$ & & \\
\hline Semantic-based Method & & & & & & \\
\hline Ontology-based Method & & & & $\mathrm{X}$ & & \\
\hline Regression-based Method & & & & & & \\
\hline Uses Sentiment & & & & & & \\
\hline Generation of new Data Corpus & & & $\mathrm{X}$ & & & \\
\hline Generation of new Lexicon & & & & & & \\
\hline Works with English Language & $\mathrm{X}$ & $\mathrm{X}$ & $\mathrm{X}$ & $\mathrm{X}$ & $\mathrm{X}$ & $\mathrm{X}$ \\
\hline Works with Other Languages & & & & & & \\
\hline Detects Single Emotion & $\mathrm{X}$ & $\mathrm{X}$ & $\mathrm{X}$ & $\mathrm{X}$ & $\mathrm{X}$ & $\mathrm{X}$ \\
\hline Detects Multiple Emotions & & & & & & \\
\hline Word-level Detection & & & $\mathrm{X}$ & & $\mathrm{X}$ & $\mathrm{X}$ \\
\hline Sentence-level Detection & $\mathrm{X}$ & $\mathrm{X}$ & & & & \\
\hline Document-level Detection & & & & & & $\mathrm{X}$ \\
\hline Emotion Intensity Detection & & & & & & \\
\hline Personality Detection & & & & & & \\
\hline Emotion-Cause Detection & & & & & & \\
\hline Public Mood Detection & & & & & & \\
\hline Temporal Effect & & & & & & \\
\hline & & & & & & \\
\hline & & & & & & \\
\hline
\end{tabular}

Table 2.15: Features used in Existing Works - Table III. 


\begin{tabular}{|c|c|c|c|c|c|c|}
\hline Features & $\begin{array}{c}\text { Mohammad } \\
\text { et al. } \\
{[60]}\end{array}$ & $\begin{array}{c}\text { Summa } \\
\text { et al. } \\
{[61]}\end{array}$ & $\begin{array}{l}\text { Sen } \\
\text { et al. } \\
{[62]}\end{array}$ & $\begin{array}{l}\text { Jain } \\
\text { et al. } \\
{[63]}\end{array}$ & $\begin{array}{l}\text { Lee } \\
\text { et. al. } \\
{[67]}\end{array}$ & $\begin{array}{c}\mathrm{Li} \\
\text { et. al. } \\
{[68]}\end{array}$ \\
\hline \multicolumn{7}{|l|}{$\overline{\text { Keyword-based }}$} \\
\hline Lexicon-based & $\mathrm{X}$ & & & & & \\
\hline Learning-based & & $\mathrm{X}$ & $\mathrm{X}$ & $\mathrm{X}$ & & $\mathrm{X}$ \\
\hline Supervised Machine Learning-based & & & $\mathrm{X}$ & $\mathrm{X}$ & & $\mathrm{X}$ \\
\hline Support Vector Machine & & & $\mathrm{X}$ & $\mathrm{X}$ & & \\
\hline Naive Bayes & & & & $\mathrm{X}$ & & $\mathrm{X}$ \\
\hline \multicolumn{7}{|l|}{ Bayesian Models } \\
\hline \multicolumn{7}{|l|}{ Decision Tree } \\
\hline \multicolumn{7}{|l|}{ K-Nearest Neighbor } \\
\hline Neural Networks & & & $\mathrm{X}$ & & & \\
\hline \multicolumn{7}{|l|}{ Maximum Entropy } \\
\hline \multicolumn{7}{|l|}{ Majority Classifier } \\
\hline \multicolumn{7}{|l|}{ Unsupervised Machine Learning-based } \\
\hline Semi-supervised Machine Learning-based & & $\mathrm{X}$ & & & & \\
\hline \multicolumn{7}{|l|}{ Hybrid Method } \\
\hline \multicolumn{7}{|l|}{ Ensemble Method } \\
\hline \multicolumn{7}{|l|}{ Fuzzy-Logic } \\
\hline Rule-based Method & & & & & $\mathrm{X}$ & \\
\hline \multicolumn{7}{|l|}{ Reasoning Method } \\
\hline \multicolumn{7}{|l|}{ Semantic-based Method } \\
\hline \multicolumn{7}{|l|}{ Ontology-based Method } \\
\hline Regression-based Method & $\mathrm{X}$ & & & & & \\
\hline Uses Sentiment & & & $\mathrm{X}$ & & & \\
\hline Generation of new Data Corpus & $\mathrm{X}$ & & & & & \\
\hline \multicolumn{7}{|l|}{ Generation of new Lexicon } \\
\hline Works with English Language & $\mathrm{X}$ & $\mathrm{X}$ & $\mathrm{X}$ & $\mathrm{X}$ & & \\
\hline Works with Other Languages & & & & $\mathrm{X}$ & $\mathrm{X}$ & $\mathrm{X}$ \\
\hline Detects Single Emotion & $\mathrm{X}$ & $\mathrm{X}$ & $\mathrm{X}$ & $\mathrm{X}$ & $\mathrm{X}$ & $\mathrm{X}$ \\
\hline \multicolumn{7}{|l|}{ Detects Multiple Emotions } \\
\hline Word-level Detection & $\mathrm{X}$ & $\mathrm{X}$ & $\mathrm{X}$ & $\mathrm{X}$ & $\mathrm{X}$ & $\mathrm{X}$ \\
\hline \multicolumn{7}{|l|}{ Sentence-level Detection } \\
\hline \multicolumn{7}{|l|}{ Document-level Detection } \\
\hline Emotion Intensity Detection & $\mathrm{X}$ & & & & & \\
\hline \multicolumn{7}{|l|}{ Personality Detection } \\
\hline Emotion-Cause Detection & & & & & $\mathrm{X}$ & $\mathrm{X}$ \\
\hline Public Mood Detection & & & & & & \\
\hline Temporal Effect & & & & & & \\
\hline
\end{tabular}

Table 2.16: Features used in Existing Works - Table IV. 


\begin{tabular}{|c|c|c|c|c|}
\hline Features & $\begin{array}{c}\text { An } \\
\text { et. al. } \\
{[69]}\end{array}$ & $\begin{array}{c}\text { Kanger } \\
\text { et. al. } \\
{[76]}\end{array}$ & $\begin{array}{c}\text { Grover } \\
\text { et. al. } \\
{[77]}\end{array}$ & $\begin{array}{c}\text { Joshi } \\
\text { et. al. } \\
{[78]}\end{array}$ \\
\hline Keyword-based & & & $\mathrm{X}$ & \\
\hline Lexicon-based & & & & $\mathrm{X}$ \\
\hline Learning-based & $\mathrm{X}$ & $\mathrm{X}$ & $\mathrm{X}$ & \\
\hline Supervised Machine Learning-based & $\mathrm{X}$ & $\mathrm{X}$ & & \\
\hline Support Vector Machine & & & $\mathrm{X}$ & \\
\hline Naive Bayes & $\mathrm{X}$ & & $\mathrm{X}$ & \\
\hline \multicolumn{5}{|l|}{ Bayesian Models } \\
\hline \multicolumn{5}{|l|}{ Decision Tree } \\
\hline \multicolumn{5}{|l|}{ K-Nearest Neighbor } \\
\hline Neural Networks & & $\mathrm{X}$ & & \\
\hline \multicolumn{5}{|l|}{ Maximum Entropy } \\
\hline \multicolumn{5}{|l|}{ Majority Classifier } \\
\hline \multicolumn{5}{|l|}{ Unsupervised Machine Learning-based } \\
\hline \multicolumn{5}{|l|}{ Semi-supervised Machine Learning-based } \\
\hline Hybrid Method & & & $\mathrm{X}$ & \\
\hline \multicolumn{5}{|l|}{ Ensemble Method } \\
\hline Fuzzy-Logic & & $\mathrm{X}$ & & \\
\hline Rule-based Method & & $\mathrm{X}$ & $\mathrm{X}$ & $\mathrm{X}$ \\
\hline \multicolumn{5}{|l|}{ Reasoning Method } \\
\hline \multicolumn{5}{|l|}{ Semantic-based Method } \\
\hline \multicolumn{5}{|l|}{ Ontology-based Method } \\
\hline \multicolumn{5}{|l|}{ Regression-based Method } \\
\hline \multicolumn{5}{|l|}{ Uses Sentiment } \\
\hline \multicolumn{5}{|l|}{ Generation of new Data Corpus } \\
\hline \multicolumn{5}{|l|}{ Generation of new Lexicon } \\
\hline Works with English Language & $\mathrm{X}$ & $\mathrm{X}$ & & $\mathrm{X}$ \\
\hline Works with Other Languages & $\mathrm{X}$ & & $\mathrm{X}$ & \\
\hline Detects Single Emotion & $\mathrm{X}$ & $\mathrm{X}$ & $\mathrm{X}$ & $\mathrm{X}$ \\
\hline \multicolumn{5}{|l|}{ Detects Multiple Emotions } \\
\hline Word-level Detection & $\mathrm{X}$ & $\mathrm{X}$ & $\mathrm{X}$ & $\mathrm{X}$ \\
\hline Sentence-level Detection & & & & $\mathrm{X}$ \\
\hline \multicolumn{5}{|l|}{ Document-level Detection } \\
\hline \multicolumn{5}{|l|}{ Emotion Intensity Detection } \\
\hline \multicolumn{5}{|l|}{ Personality Detection } \\
\hline \multicolumn{5}{|l|}{ Emotion-Cause Detection } \\
\hline \multicolumn{5}{|l|}{ Public Mood Detection } \\
\hline Temporal Effect & & & & $\mathrm{X}$ \\
\hline
\end{tabular}

Table 2.17: Features used in Existing Works - Table V. 


\subsubsection{Emotion and Sentiment Detection Methods}

Several approaches exist for textual emotion and sentiment detection. Emotion and sentiment recognition task is a part of Affective Computing and the computational methods used in this area have been classified into several categories by various researchers. The methods defined by these researchers can be generalized into four categories - 'Keyword-based Method', 'Lexicon-based Method', 'Machine learning Method' and 'Hybrid Method'. Existing works utilized unigrams (one word), n-grams (multiple words), emotiocons, hashtag words, punctuations, negations as features for the emotion detection task. Approaches for textual emotion detection are defined as described in the following subsections.

\section{Keyword-based Method}

Keyword-based emotion detection is the most intuitive and straightforward approach. The idea is to find out patterns similar to emotion keywords and match them. The first task is to find out the word which expresses the emotion in a sentence. This is normally done by tagging the words of a sentence with Parts-Of-Speech tagger and then extracting the Noun, Verb, Adjective and Adverb (NAVA) words. Most linguistic and emotion based researches mentioned that these are the most probable emotion carrying words $[65,66,70]$. Then these words are matched against a list of words representing emotions according to a specific emotion model. Whichever emotion matches with the keyword is considered as the emotion of the specific sentence. If the word matches with multiple emotions from the list, then different approaches can be applied. In some keyword-dictionaries, each word has a probability score for each emotion and the emotion with the highest probability is picked as the emotion of the word. In some other works, the first emotion matched with the word is picked as the primary emotion of the word. For the reference list of keyword or the keyword dictionary, it differs depending on the researcher. The keyword dictionary is normally made by the

researchers based on the emotions and the words they are working with. There are online 
tools and programs like WordNet[74] which can find synonyms and antonyms of words which can be used to make the dictionary.

\section{Lexicon-based Method}

Lexicon-based emotion detection approach classifies text using a lexicon (a knowledge-base with text labeled according to emotions) appropriate for the input dataset[59, 60]. Emotion detection is similar to the previous method, but in this case, an emotion lexicon is used instead of the word list. National Research Council Canada(NRC), EmoSenticNet(ESN) are some mostly used emotion and sentiment lexicons[75].

\section{Machine learning Method}

Both 'supervised' $[57,62]$ and 'unsupervised' $[65,70]$ machine learning methods are used for textual emotion detection in which a model is designed to train a classifier with a part of the dataset and then test the classifier with the rest of the data. For supervised method, an annotated emotion dataset is used for training and testing the supervised classifier. Naive Bayes, Support Vector Machine, and Decision Tree may be named as the mostly used classifiers. By the definition of 'Unsupervised Classification', the data used is not labeled with the classes. The classifier starts with several seed words for each emotion which are then cross-referenced to the sentences, thus classifying the sentences to corresponding emotions. This trains the classifier model which is then further used to label the testing data. Unsupervised method is a more generalized one but in most cases, supervised classification achieves better accuracy. 


\section{Hybrid Method}

Hybrid approach for emotion detection in text combines any two or all three methods defined to achieve the benefit of multiple methods and reach the maximum level of accuracy. In some previous works, it was proved that applying a combination of multiple emotion detection methods gives better results than individual methods[54, 66].

According to the previous surveys $[37,51,54,52,53]$ done on comparison between the accuracy of emotion detection using Keyword-based Method, Lexicon-based Method, Machine learning Method and Hybrid Method, it was mentioned that Keyword-based Method and Lexicon-based Method worked better. Machine learning Method worked better with larger datasets and Hybrid Method had similar accuracy to Machine learning Method. Although these methods are the benchmark approaches for textual emotion detection, some researchers tried natural language processing[58], linguistic rule-based methods[76], ensemble of multiple methods[13] or some novel methods almost completely unique and achieved some good results. Modifications to those novel methods may lead to an improved and highly accurate automatic emotion detection system from text input.

\subsubsection{Datasets}

In the literature, different existing or customized datasets have been used for emotion detection according to the types of the experiments of different researchers. The annotated datasets are annotated with particular emotion models, hence bearing only those emotions that exist in the specific emotion model. A small number of emotion labeled datasets are available. Some researchers contributed some data corpus that they built to utilized in their experiments. But still the number of available datasets for emotion analysis is not enough. Further, there is no generalized dataset which can be used for any emotion model. Researchers are either bound to use the emotion model that exists in the datasets or to 


\begin{tabular}{|c|c|c|}
\hline Dataset & Datasize & Description \\
\hline \hline EmoBank[79] & 10K Sentences & $\begin{array}{c}\text { Double annotation with valence, arousal } \\
\text { and dominance were used from the } \\
\text { perspectives of both writer and reader }\end{array}$ \\
\hline $\begin{array}{c}\text { The Valence and } \\
\text { Arousal Facebook } \\
\text { Posts[80] }\end{array}$ & $\begin{array}{c}2895 \text { Facebook } \\
\text { posts }\end{array}$ & $\begin{array}{c}\text { Double annotation with } \\
\text { valence and arousal values }\end{array}$ \\
\hline $\begin{array}{c}\text { The Emotion in } \\
\text { Text data set[81] }\end{array}$ & 40000 Tweets & $\begin{array}{r}\text { Annotated with Anger, Boredom, Empty, Enthusiasm, } \\
\text { Fun, Happiness, Hate, Love, Relief, } \\
\text { Sadness, Surprise, Worry, Neutral }\end{array}$ \\
\hline EmoLex[82] & 14182 Unigrams & $\begin{array}{r}\text { Annotated with Sentiments- Negative, Positive and } \\
\text { Emotions- Anger, Anticipation, Disgust, } \\
\text { Fear, Joy, Sadness, Surprise, Trust }\end{array}$ \\
\hline ISEAR[83] & 7666 Sentences & $\begin{array}{c}\text { Contains responses of questionnaires } \\
\text { on seven emotions (Joy, Fear, Anger, } \\
\text { Sadness, Disgust, Shame, and Guilt) } \\
\text { from 37 countries from 5 continents }\end{array}$ \\
\hline Affective Text[84] & $\begin{array}{c}\text { Annotated with 6 basic emotions } \\
\text { from Ekman's model and polarity }\end{array}$ \\
\hline
\end{tabular}

Table 2.18: Datasets

generate a data corpus and then manually annotate the data according to emotion labels. The latter task is time consuming. Some of the most commonly used datasets in recent works are listed in Table 2.18 together with a brief description of the amount of data that exists in each dataset.

\subsubsection{Influential Users in Social Network}

Finding influential people and groups in any social network can be significant to detect and control the information flow. Haewoon et. al[85] analyzed Twitter and generated the first quantitative study on Twitter. They crawled Twitter on July 2009 and got more than 41 million user information along with the following/follower relations. They computed the distributions of users, their followers, the people they were following, the recent trends, separation, homophily and diffusion of information in Twitter. Their analysis showed noticeable differences between Twitter and other social networks. Because of the one directional relation 
between users in Twitter, it defied the power law and the distributions were not very structured. The list of top ranked users also differed according to the method of the computationnumber of followers, page rank, number of retweets. They also mentioned and calculated the active periods of trends which was less than a week in most cases. Their retweet trees and temporal analysis of retweets showed the diffusion of information and it's difference from other media. Vosoughi et. al[86] showed how both true and false news spread in Twitter network. According to their observation, false news spread more than true news and false news tend to be unique. Among various kinds of false news, rumors regarding political issues spread quickly among huge number of people rather than other types of rumors.

Riquelme et. al[87] conducted a detailed survey on Twitter activities, influences and popularity of the users. They showed a classification of different measures of Twitter data based on various metrics and contents. The relationships between users and tweets was discussed in detail. Twitter metrics and their time complexity were explained with detail anatomy of Twitter. They mentioned existing methods of finding influential users and experiments done on Twitter data. Finally, some open research problems were stated for researchers to analyze. T-PICE[88] is an influential community extraction system which identifies influential people by classifying people into some personality classes based on their tweets on a specific topic. NavigTweet[89] is a visualization tool which finds the influential people from the friend list of an user by analyzing the contents of tweets that are common between them. An analysis on the tweets about two car companies to find out the influential users led to some hypotheses in [90]. It represented some interesting patterns of influential people of a social network and some of the textual characteristics like their use of words, hashtags, links, expressions etc. Emotion contagion in Facebook was analyzed by Coviello et. al[91]. Facebook posts were analyzed using instrumental variable regression method to detect positive and negative emotions of people in rainy days and the effect of one's emotion on his/her friends' emotion. Detecting influential people from a social network is a very lucrative research area for referral 
marketing to spread the information of any product and reach highest possible nodes of a network $[92]$.

In most of the previous works, researchers counted the number of followers to find out the influential users from Twitter. The idea was that "the more follower a person has, the more influential he/she is". Some researchers ranked the users based on their total number of tweets[93] or summation of total number of tweets and the number of retweets of those tweets[94]. To find the most influential users on a specific topic, the number of tweets, the number of replies by the author, the number of retweets on a specific topic were used and normalized with the total number of tweets on that topic[95]. Followers and following counts were also used to find the influential users. The ratio of number of followers and followees[96], ratio of followers and summation of followers and followees $[93,87]$ were used to compute how influential a user is in Twitter. In [97], the reciprocal relationship between users were also counted in case the user had less followers than the number of people the user followed.

Acquaintance Affinity Identification(AAI) score was calculated with the number of followers, number of users mentioning the author, number of users replied to that author's tweets, number of users retweeted the author's tweets[98]. Various common centrality measures like betweenness and closeness centrality were used by researchers to find influential users from a network[99], whereas few researchers found their own centrality indexes like H-index which considered a maximum h value for replies, retweets, likes for the calculations[100, 101]. Google's page rank algorithm was modified as TunkRank[102] and UserRank[103] using followers, followees and tweets to rank Twitter users. Lahuerta-Otero et. al[90] used regression model with lexical diversities in tweets, average number of characters used in tweets, hashtags, user mentions, URLs, followees, positive and negative sentiments to extract influential users from Twitter. Exponential of number of followers was used in [104] to find the popular users from Twitter. Users were ranked using both tweet-based and user-based scores by 
Francalanci et. al[89]. They used favorites, followers, following, listed, tweets for user-based score and favorited, retweets, urls, hashtags, mentions for tweets-based score. Each parameter was assigned with weights based on Analytic Hierarchy Process (AHP).

\subsection{Few Challenges or Open Problems}

Emotion and sentiment analysis is a research field with lots of unturned stones and possibilities. Due to the diverse nature of human emotions, there are still lots of scopes for developing unique systems or improving and enriching existing systems with effective modifications and capabilities. Some possible future directions in the research of emotion and sentiments detection and analysis from texts are listed as follows.

\subsubsection{Text-based Emotion Network Detection}

The existing research works based on textual emotion in social networks mostly focused on the post by a user. The emotions of the comments or replies to those posts can give us an idea about the similarity and dissimilarity between emotions of users in a network. This can be used to detect emotion networks within the social networks and the propagation of particular emotions.

\subsubsection{Emotion Intensity Detection}

Considering the amount of research going on about emotion detection, a very small number

of researchers are concentrating on intensity of the detected emotion[60]. Each emotion may have different intensity level and detecting the intensity of an emotion can be beneficial for emotion analysis. For example, if a person writes "I am sad" or "I want to end my life, there 
is nothing left for me", then the existing systems will label both of these declarations as 'sad' emotions. However, in reality, the intensity of sadness in both statements is different - the former represents normal sadness while the other reflects severe depression or suicidal emotion.

\subsubsection{Sarcasm Detection}

Sarcasm detection from text is a very complex task and the maximum accuracy achieved so far is not very much. Correct sarcasm detection depends on correctly using sentence structure, detecting accurate emotion behind the sentence, understanding the context of the sentence, and many other parameters. For example, "I'm trying to imagine you with a personality" [105] represents a sarcasm which is very implicit and hard to detect for an existing automatic sarcasm detection system.

\subsubsection{Multiple Emotion Detection}

In most of the emotion detection efforts, researchers concentrate on the primary emotion in the text. Sentences expressing multiple emotions are either discarded or labeled with the

first emotion that was detected. For example, if a person writes "I was sad this morning but now I am happy" or "This makes me happy and sad at the same time", then the system should be able to recognize both emotions with a temporal and/or spatial dimension.

\subsubsection{Emotion-Cause Detection}

A limited number of works focus on the cause of the emotion that was expressed by a person$[67,68]$. Sometimes, detecting the cause can increase the accuracy of the detection 
of the correct emotion in a text or even speech. For example, if a person expresses "I am so happy!!! It is raining!!", then the system should recognize that the reason of his/her happiness is rain. This can also be applied to personalized systems where the system has knowledge of the likes and dislikes of a person and can detect the cause of an emotion even if the cause is not directly stated in the same sentence.

\subsubsection{Personality or Mood Detection}

By detecting the emotion from text for a particular person, his/her personality or mood can be detected and analyzed[15]. Personality or mood detection can be integrated into existing social networking platforms and other applications for personalized suggestions. For example, if a person is angry and is expressing anger in his/her text, then an application may suggest to him/her different things he/she likes (i.e. a nearby restaurant which serves his/her favorite food, a nearby movie theater where a new movie is playing which is of his/her favorite genre, and so on).

\subsubsection{Emotion versus Individual or Social Parameters}

Human emotions can be related to their age, gender, time, location, ethnicity, political views, educational qualifications, in addition to some other individual, social, temporal and spatial parameters. The connection between emotion and these parameters can present some new

dimensions for emotion analysis based research[2]. For example, adult people are in general more concerned about political issues than teenagers. Issues in one country may effect people of neighbor countries and other countries of the world in different ways.

In this research, the problems of detecting the emotions and sentiments of Twitter users from their tweets and replies and finding their influence score in the network was addressed. 
One of the mostly used supervised classifier, 'Naive Bayes' was applied for classifying the emotions and sentiments of texts. Then the influential users on a specific topic were extracted by combining the parameters of user and his/her tweets and replies. Few parameters from the existing works were included and at the same time, few new parameters were introduced that were applicable for the calculations. 


\section{Chapter 3}

\section{Text-Based Analysis of Emotion by Considering Tweets}

This chapter is based on the emotion detection and analysis work in [2]. In this work, after extracting tweets on various topics, they were preprocessed and each tweet was divided into words and then corresponding parts-of-speeches were identified for those words. After analyzing the words, they were classified into 32 emotion classes according to Robert Plutchik's emotion classification model[41]. Emotions were then analyzed with respect to gender and location of the user and the time of the tweet. The analysis revealed some interesting outcomes and some new challenges. The rest of this chapter is outlined as follows. Subsection 3.1 describes the methodology of the emotion classification process. Subsection 3.2 shows experimental results and analysis of the results. Subsection 3.3 concludes the work and outlines some possible future directions.

\subsection{Tweet Emotion Detection}

The process for detecting emotions from tweets and analyzing them with respect to user's gender and location involves several sequential steps. Tweets should be preprocessed before 
using them in the emotion analysis model. After that, parts-of-speech tagging is necessary to find out which words from the tweets to focus on. An emotion dictionary is required according to the chosen emotion class model. After detecting emotion unigrams, they should be classified into corresponding emotion classes.

\subsubsection{Preprocessing}

Collected tweets contained considerable amount of noises (e.g., extra and missing symbols, characters, spelling mistakes). The dataset was preprocessed by removing unnecessary parts in order to have more usability. First, @username (e.g., @mary), URLs (e.g., http://a.com/) and emoticons (e.g., ':-)') were removed from the dataset. Then, multiple

dots (e.g., ....), symbols (e.g., !!!), characters (e.g., happyyyyy) were also removed. Hashtag symbols were removed without removing the words associated with them (e.g., in the hashtag \#sad - only \# was removed, not 'sad'). After removing these symbols, the dataset was checked for redundancy. Finally, the filtered dataset had tweets with no emoticons, hashtags, unnecessary multiple occurrences of one symbol or illegible words.

\subsubsection{POS Tagging}

A 'parts-of-speech tagger' was used to tag each word of the tweets into corresponding parts-ofspeech. In total, 36 parts-of-speech classes were used. They were- Coordinating conjunction, Cardinal number, Determiner, Existential there, Foreign word, Preposition or subordinating conjunction, Adjective, Adjective - comparative, Adjective - superlative, List item marker, Modal, Noun - singular or mass, Noun - plural, Proper noun - singular, Proper noun plural, Predeterminer, Possessive ending, Personal pronoun, Possessive pronoun, Adverb, Adverb - comparative, Adverb - superlative, Particle, Symbol, to, Interjection, Verb - base form, Verb - past tense, Verb - gerund or present participle, Verb - past participle, Verb - 
non 3rd person singular present, Verb - 3rd person singular present, Wh determiner, Wh pronoun, Possessive wh pronoun), Wh adverb and Others. Tweet words were tagged and each word was stored in one of those 37 (36 POS and Others) classes against user IDs.

\subsubsection{Emotion Dictionary}

Existing emotion detection systems use different emotion models according to their requirements. As described in the literature, Ekman's model[38], Johnson-laird and Oatley model[40] and Circumplex model[42] are the mostly used emotion classification models. But the limitation of these models is that they focus on only few basic emotions. The Circumplex model works with 28 emotions and it is a widely used emotion model. Another emotion model called 'Plutchik's Wheel of Emotion'[41] defined 32 emotions. Emotion classification used in this work was Plutchik's model with 32 emotions (as shown in Fig.3.1) - Acceptance, Admiration, Aggressiveness, Amazement, Anger, Annoyance, Anticipation, Apprehension, Awe, Boredom, Contempt, Disapproval, Disgust, Distraction, Ecstasy, Fear, Grief, Interest, Joy, Loathing, Love, Optimism, Pensiveness, Rage, Remorse, Sadness, Serenity, Submission, Surprise, Terror, Trust, and Vigilance.

An interesting observation about tweets is that some of them did not express any specific emotion; they just expressed some facts or asked some questions. Some tweets represented taunts, requests, advises, suggestions or sarcasms. Some tweets had words from multiple languages. Some tweets did not make any sense, they looked like just some random words put together. Those tweets were classified as Others.

The emotion dictionary was built based on these 32 emotions. Synonyms for each emotion word were collected from various online English dictionaries[106, 25, 107, 108, 109]. After inserting the synonyms from English dictionaries, some common synonymous words 


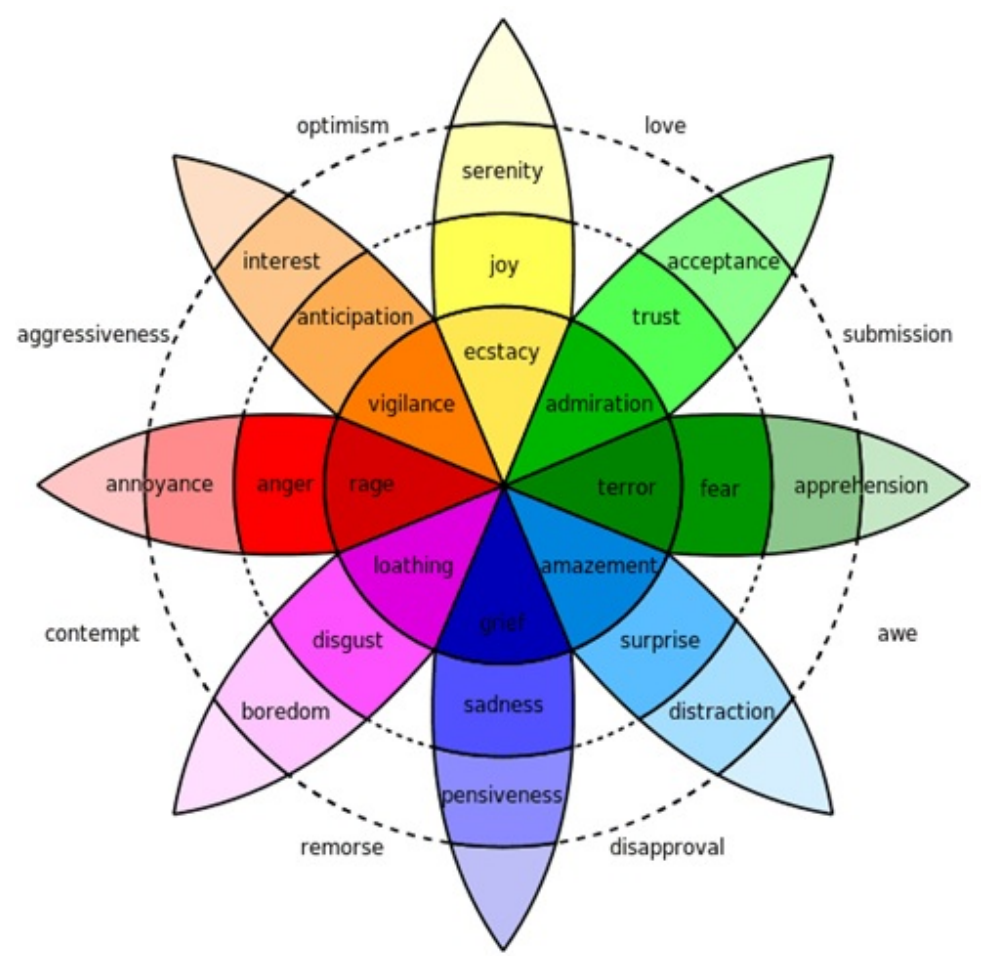

Figure 3.1: Plutchik's Wheel of Emotion.

used in tweets were collected from some dictionaries based on tweet words only[110, 111]. Those words were also inserted into the emotion dictionary for each emotion. Finally some common acronyms used nowadays were extracted from different sources and were inserted for each emotion. After merging all these synonymous words and acronyms, the emotion dictionary had more than 20 synonyms for each emotion.

\subsubsection{Unigram Selection and Dataset Annotation}

After building the emotion dictionary, the dataset was annotated with emotions. To select emotion unigrams (one word), all unnecessary POSs were removed from the dataset (i.e., articles, prepositions, etc.) and the concentration was on the rest of the words to annotate each tweet with an emotion. Nouns, adjectives and adverbs were the POSs that were used. For tweets in which multiple emotions existed, the primary emotion was considered and 
the tweet was annotated accordingly. The emotion model used in this work has not been previously applied in any other work, so no automated system for data annotation exists according to this model. Therefore, the dataset annotation was done manually.

\subsubsection{Emotion Classification}

The final step for emotion detection was emotion classification by a classifier using the emotion dictionary. Different supervised classifiers like KNN, Naive Bayes classifier, and Decision Tree have been used in literature for emotion classification. In most cases, the Naive Bayes classifier produced the most accurate results, hence was used for this work.

$$
\begin{gathered}
p\left(C_{k} \mid x_{1}, \ldots, x_{n}\right)=\frac{p\left(C_{k}\right)}{p(x)} \prod_{i=1}^{n} p\left(x_{i} \mid C_{k}\right) \\
L=\arg \max _{k \in\{1, \ldots, K\}} p\left(C_{k}\right) \prod_{i=1}^{n} p\left(x_{i} \mid C_{k}\right)
\end{gathered}
$$

Equations 1 and 2 represent the Naive Bayes classifier. Here $C_{k}$ is the emotion class. This research used 32 emotion classes. So for this case, $k$ varied from 1 to 32 and $x_{i}$ 's are the unigrams used for emotion detection from each tweet. Finally, $70 \%$ of the annotated data was used as the training set and the rest $30 \%$ data acted as the test set for the classifier.

\subsection{Experimental Results}

Every step of the methodology- preprocessing, parts-of-speech tagging, creating emotion dictionary, implementing Naive Bayes classifier, classifying tweets according to their emotions and analyzing the results were implemented and tested using different packages of $\mathrm{R}$ 
for networks, graphs, matrices, Natural Language Processing, words, parts-of-speech, and synonyms. Several data matrices and data frames were used for the intermediate calculations.

\subsubsection{Dataset}

The dataset used in this experiment was collected from the tweets of 26 October 2015[112]. The user ID, tweet location (city or some local information), user gender and time the tweets were created were extracted from the dataset. The time zone of the tweets were not considered while including the tweet creation time in the analysis. Almost $30 \%$ of the tweets had to be removed because those tweets were advertisement tweets for different products or websites. Retweets and illegible tweets were deleted from the rest of the tweets. The location of each tweet was replaced with corresponding country name based on the given city name or local information. Finally, each data in the dataset had a user ID, tweet text, gender of the user, location of the user (country) and time of the tweet expressed in 'hh:mm' format.

\subsubsection{Results}

Tweets were classified into 32 emotion classes with their corresponding ID, gender, location and time of each tweet. Users were then classified according to these parameters and analyzed based on their emotions.

\section{Emotion Network}

Shown in Figure 3.2 is the emotion network for all the 32 emotions. The network could be divided into clusters of nodes based on different emotions and nodes represent users. People who shared the same emotion are connected to each other and form one cluster. So, for each emotion, a cluster was formed with people who expressed the same emotion via their tweets. 


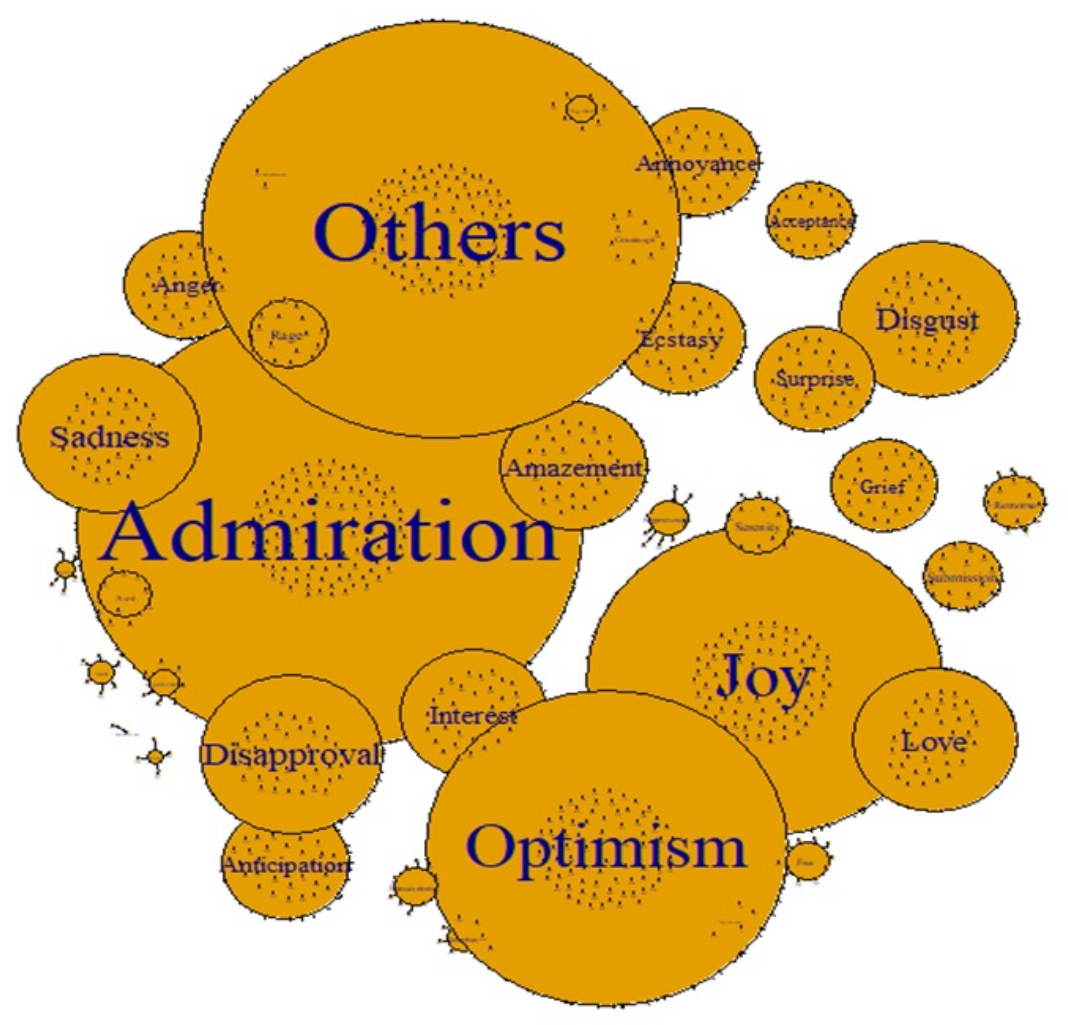

Figure 3.2: Emotion Network.

Some emotions were used more than others in tweets and this is the reason for different sized clusters. From Figure 3.2, it can be clearly realized that majority of the tweets represented Admiration, Joy, Optimism, Others. Some other notable emotions appeared in the dataset where quite a lot of users expressed Sadness, Disgust, Love, Disapproval. The rest of the emotions were encountered as well, but were not that much prominent in the dataset.

\section{Gender versus Emotion}

Gender information which was collected from the tweets was divided into two classes, namely male and female. In Figure 3.3, the $\mathrm{X}$-axis represents emotions and the $\mathrm{Y}$-axis represents percentages of tweets of that emotion from a gender. Blue bars show Female tweets, and red bars show Male tweets. 
Emotion Vs. Gender

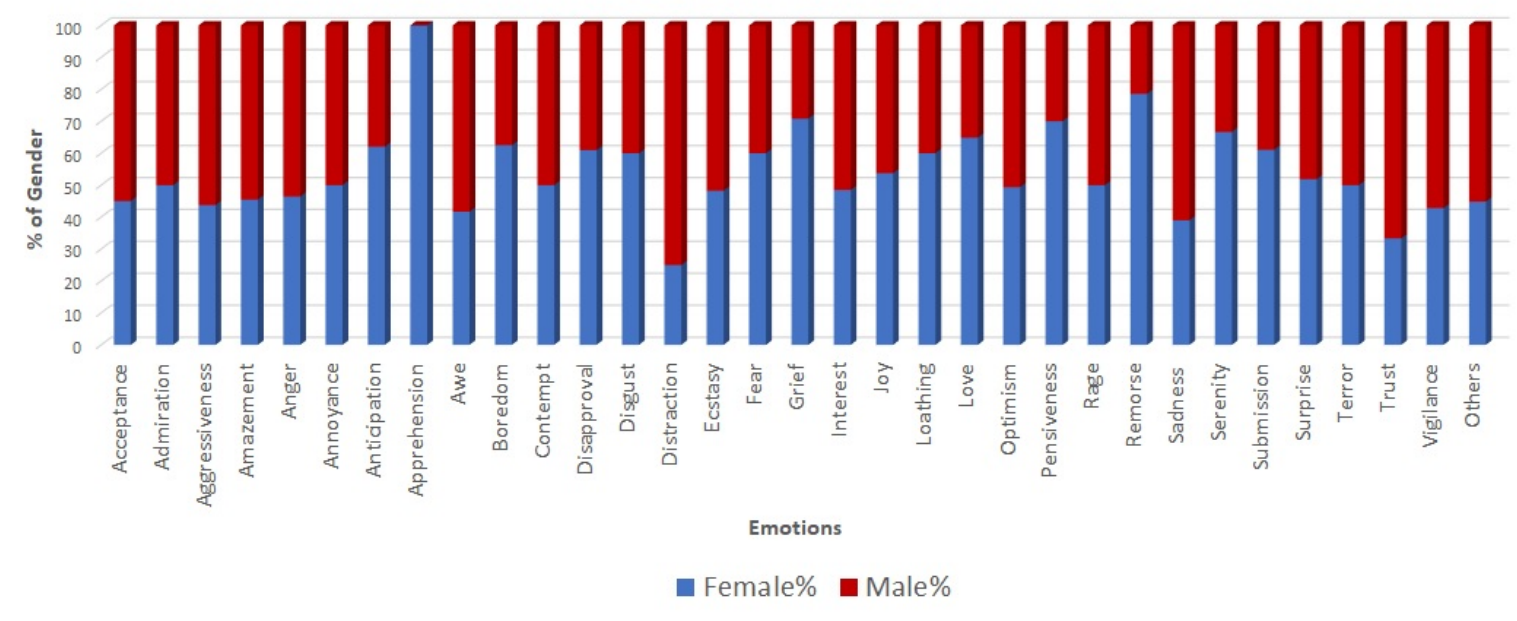

Figure 3.3: Gender Vs. Emotion.

Female users had the majority in positive emotions like Anticipation, Joy, Love, Pensiveness, Serenity, but they were also the major contributors to negative emotions like Boredom, Disgust, Disapproval, Fear, Grief, Loathing, Remorse, and Submission. In contrast, Male users expressed positive emotions like Acceptance, Amazement, Awe, and Trust and negative emotions like Aggressiveness, Anger, Distraction, Sadness, and Vigilance more than Females. Other emotions were mentioned in tweets from both Male and Female almost equally. Surprisingly, Apprehension was expressed by Females only.

\section{Time versus Emotion}

Temporal information collected from the tweets was divided into two classes. Tweets which were posted until 12:00 noon were classified as Morning tweets, and tweets that were posted after 12:00 noon were classified as Afternoon tweets. In Figure 3.4, the X-axis represents emotions and the Y-axis represents corresponding percentages of tweets based on their temporal classes. Blue bars show Afternoon tweets and red bars show Morning tweets.

From the graph shown in Figure 3.4, it is easily visible that negative emotions like 


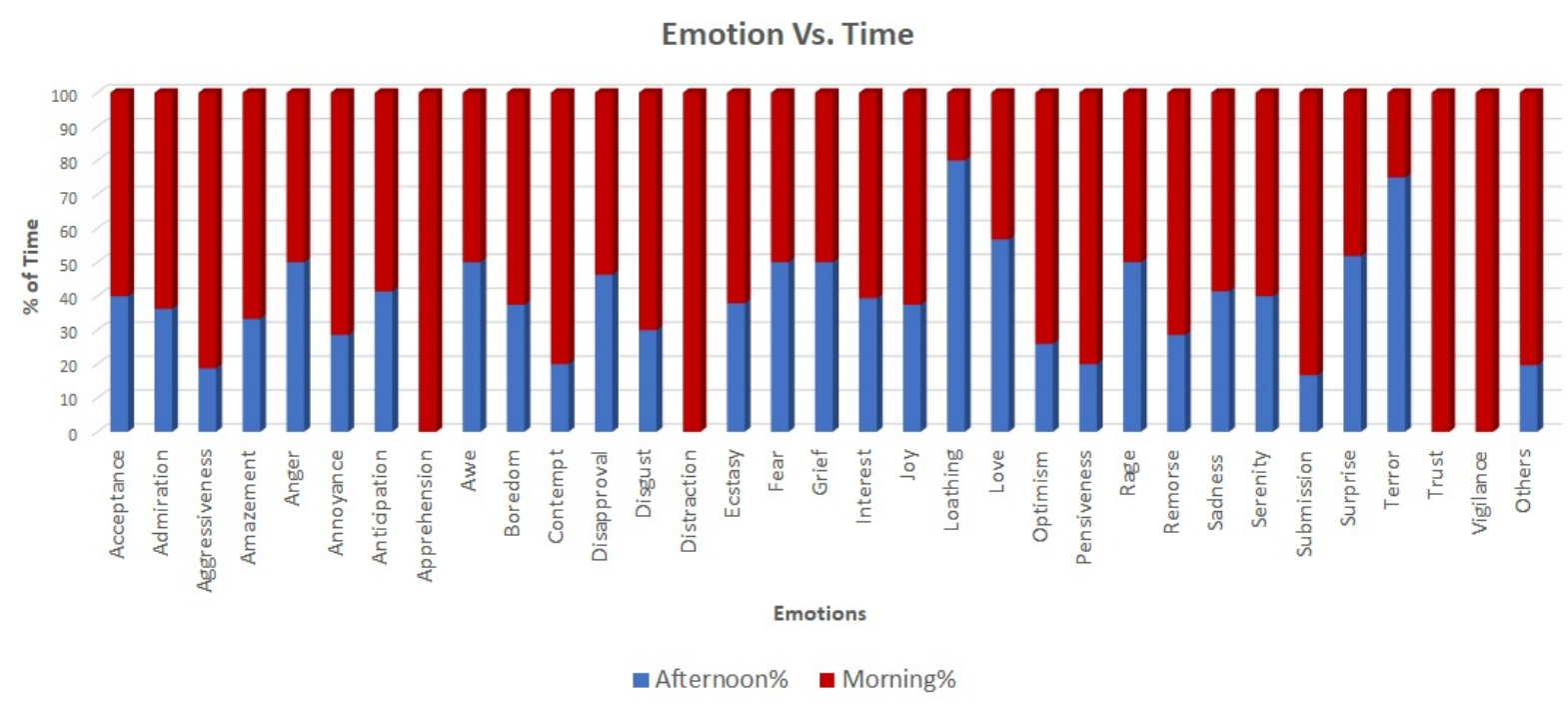

Figure 3.4: Time Vs. Emotion.

Loathing, and Terror were prominent in Afternoon tweets with one exception, namely Love.

On the other hand, positive emotions like Acceptance, Admiration, Amazement, Anticipation, Ecstasy, Interest, Joy, Optimism, Pensiveness, and Serenity; and negative emotions like Aggressiveness, Annoyance, Boredom, Contempt, Disgust, Remorse, Sadness, and Submission mostly occurred in Morning tweets. Apprehension, Distraction, Trust, and Vigilance have been encountered only in Morning tweets.

\section{Location versus Emotion}

The relationship between emotion and user location (country) is shown in Figures 3.5, 3.6, 3.7 and 3.8. In Figure 3.5, all the 32 emotions are shown in the map with different colors according to their locations. The pie charts shown in Figures 3.6-3.8 were generated based on 33 emotion classes (32 emotions and others) according to 63 countries (Africa, Argentina, Armenia, Australia, Austria, Belgium, Brazil, Canada, Chile, China, Colombia, Croatia, Czech Republic, Denmark, Dominican Republic, Ecuador, Egypt, Europe, France, Germany, Greece, Greenland, India, Indonesia, Iraq, Ireland, Israel, Italy, Japan, Kenya, Korea, Kuwait, Lebanon, Malaysia, Mexico, Morocco, Netherlands, New Zealand, Nigeria, 

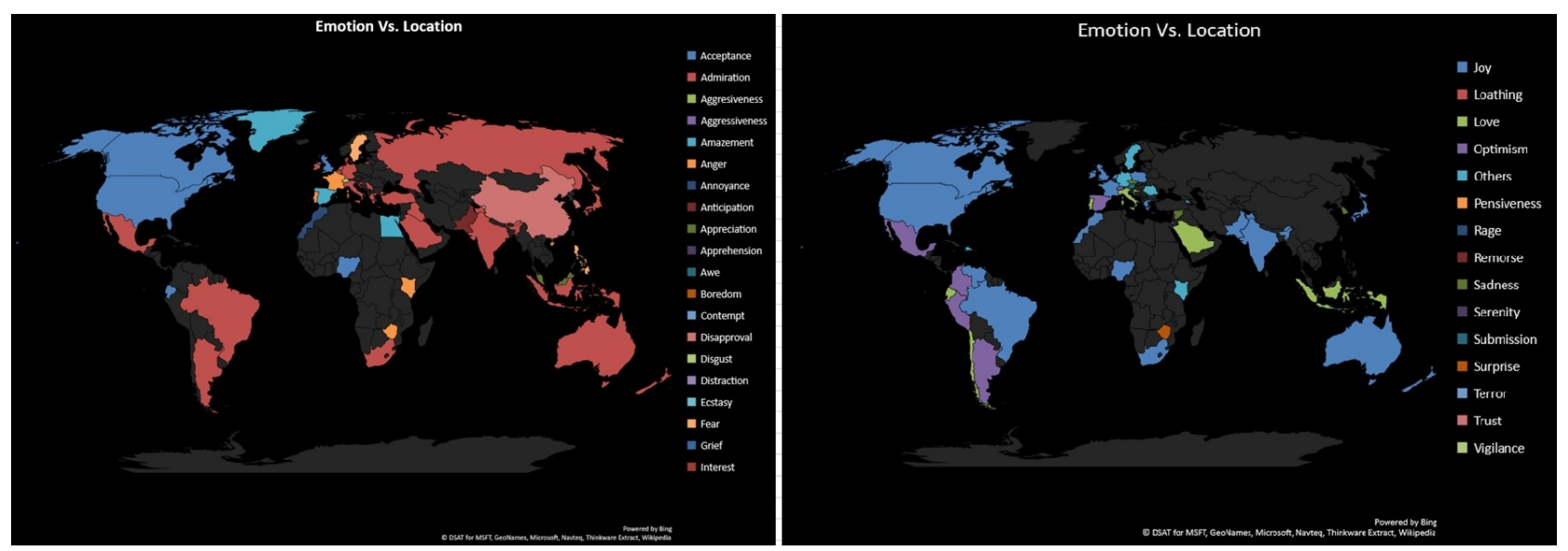

Figure 3.5: Location Vs. Emotion - Map.

Pakistan, Pennsylvania, Peru, Philippines, Poland, Polynesia, Portugal, Romania, Rome, Russia, Saudi Arabia, Serbia, Singapore, Spain, Sweden, Switzerland, Syria, Trinidad and Tobago, Turkey, UK, USA, Venezuela, Zimbabwe) which were detected from the dataset. Each circle represents an emotion and countries which contributed to that particular emotion. As most tweets were from USA and UK, these two countries were the two major contributors to all emotions.

For positive emotions like Ecstasy, Joy, Admiration, Acceptance, Anticipation, Interest, Amazement, Surprise, Pensiveness, Love, and Optimism, in addition to USA and UK, tweets from countries like Canada, Greece, Netherlands, Australia, India, and Nigeria are some major participators. Similarly, other than USA and UK, countries like Canada, Netherlands, Germany, New Zealand, Mexico, Australia, Ecuador, Europe, Indonesia, Sweden, Kenya, and Africa expressed negative emotions like Grief, Sadness, Loathing, Disgust, Boredom, Rage, Anger, Annoyance, Terror, Fear, Submission, Disapproval, Remorse, and Aggressiveness more than other countries.

Experimental result reflected some interesting aspects regarding emotions of a person with respect to his/her gender, location and time. But these results can not be generalized 

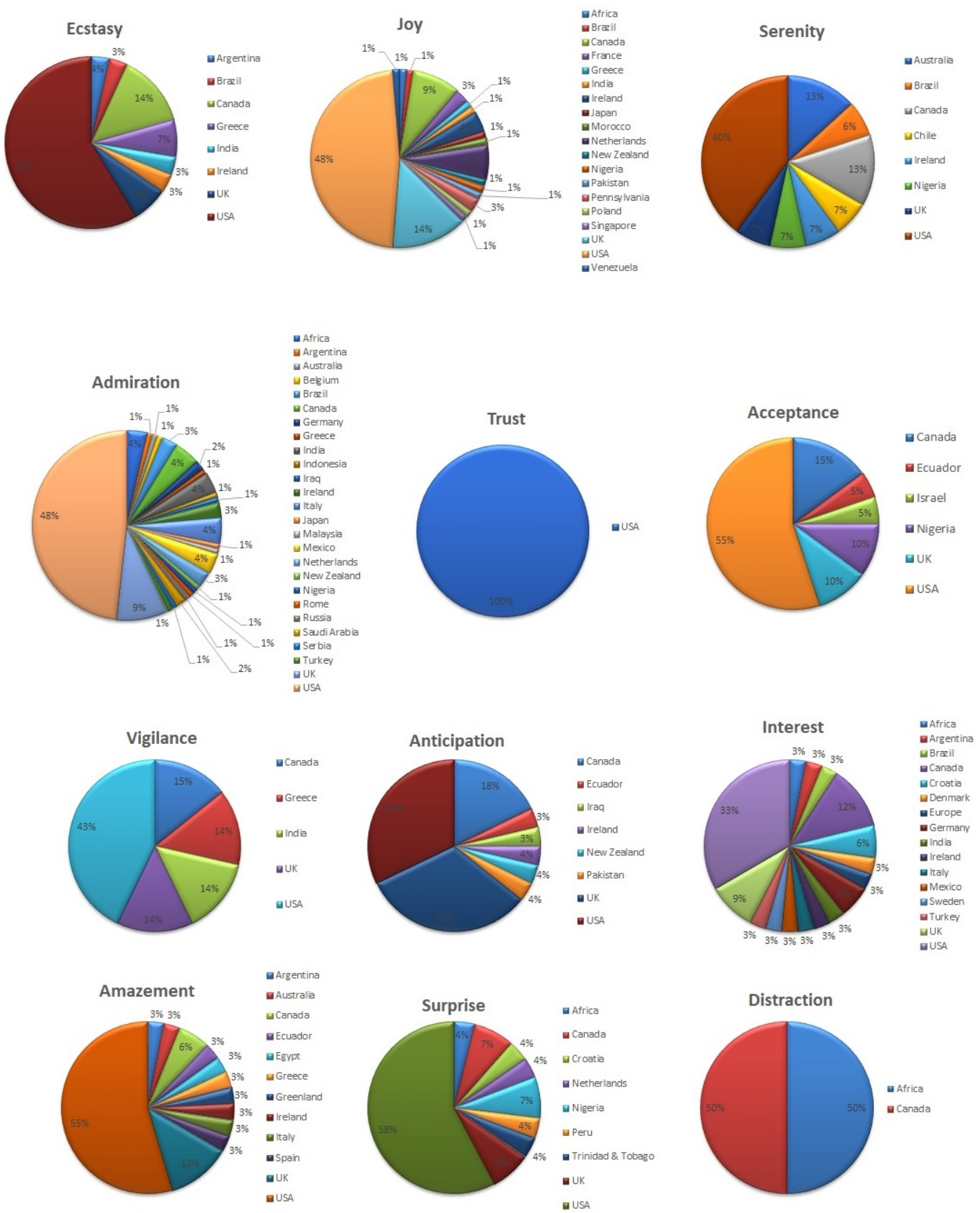

Figure 3.6: Location Vs. Emotion - Pie Chart 1. 

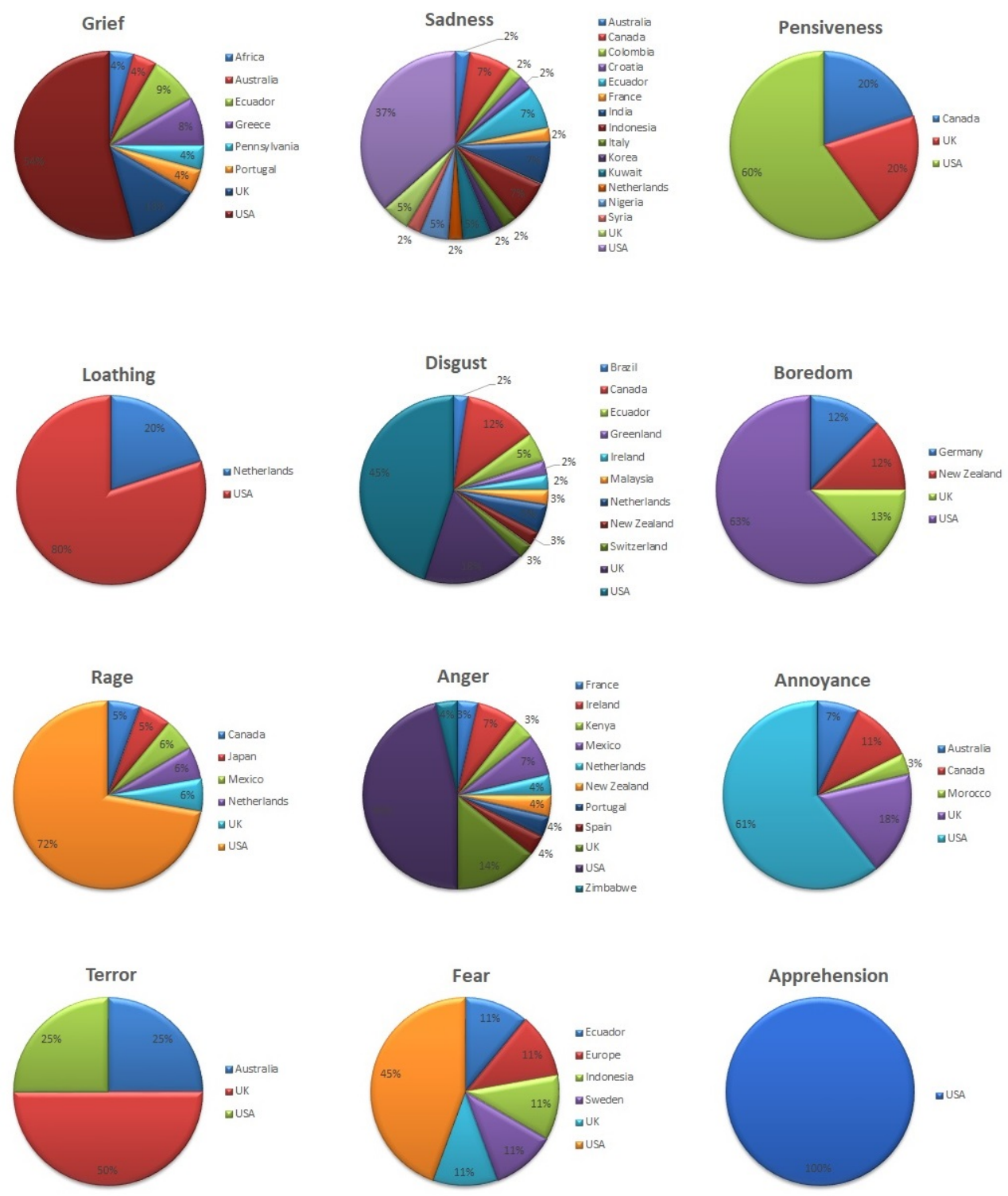

Figure 3.7: Location Vs. Emotion - Pie Chart 2. 

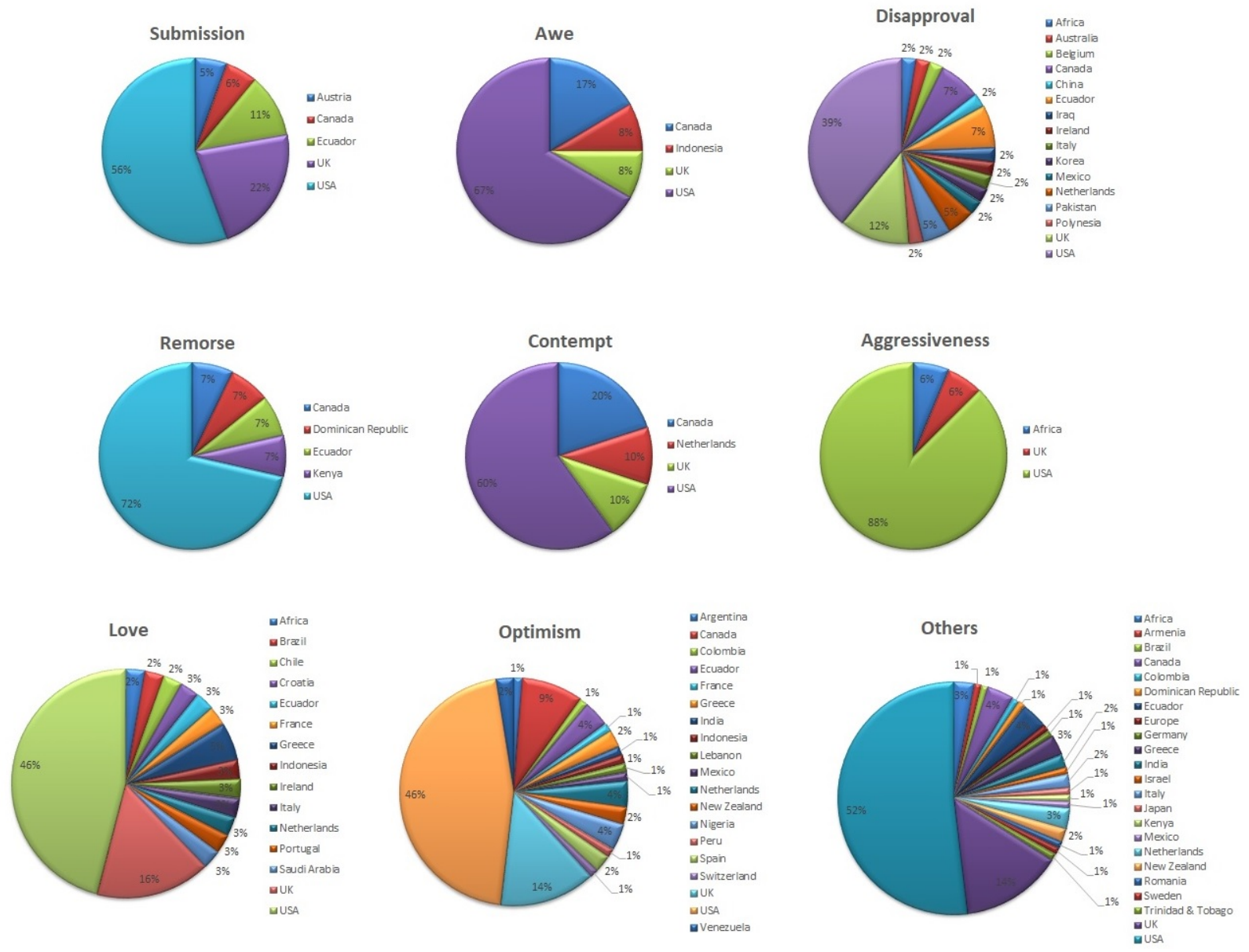

Figure 3.8: Location Vs. Emotion - Pie Chart 3. 
for all human beings and their emotions because they are completely based on a specific dataset containing tweets collected on a random day from different people scattered almost all over the world. It is important to investigate whether emotions were directly reflecting personal feelings related to any particular events associated with the time period during which the tweets were posted.

\subsection{Conclusion}

Emotion detection and analysis is an important research area for analyzing both individual and public emotions on various events. The diversity and multiple dimensions of human emotions make the process complicated and reduce the accuracy. The emotion model that was used in this work covered almost all basic human emotions. A larger emotion dictionary built by collecting words from different sources made emotion detection more sparse and accurate. Acronyms of common tweet words and some common words used in tweets only were considered in this work which made the emotion detection process easier and more efficient. After detecting emotions from the text, the analysis on the emotion with respect to gender, location and time represented some interesting human characteristics. 


\section{Chapter 4}

\section{Emotion Detection : From Data Collection to Recommendation}

Twitter is one of the most popular social network nowadays with 330 million monthly active users[113]. People express their opinions about their daily lives, different social or national or international issues. They share their views within 140 characters of text and sometimes also share audio or video files. Posts are called tweets and they are public. Other people can like posts, reply to them or retweet them. People can follow each other or can be friends with each other in Twitter. Unlike most other social networking platforms, Twitter allows one directional links, which means one user can follow another user without the latter user reciprocating the communication. These interactions lead to a network of communication containing both one-directional and bi-directional connections between the Twitter users. $\operatorname{Hashtags}(\#)$ and emojis(i.e., : $),:($, etc.) are used in tweets to enhance the sentiment of a tweet.

In this chapter, the methodology of the implementation of emotion and sentiment based recommender system is explained in detail. The methodology is different than the methodology mentioned in chapter 3 . The dataset created for this procedure including both tweets 
and replies was a new dataset which had nothing in common with the dataset that was used in chapter 3 for experiments. For emotion detection in both chapter 3 and 4, Naive Bayes classifier was used. But the features used for the classifiers in both chapters were different. In chapter 3, for Naive Bayes classification, only NAVA words of the dataset of chapter 3 were considered whereas both the full texts and NAVA words of the new dataset of chapter 4 were considered for Naive Bayes classification features in this chapter. The methodology followed from data collection to recommendation generation were explained in the following sections.

\subsection{Data Collection}

The dataset used for this experiments contains a collection of tweets, replies, retweets and their user information. The existing datasets of Twitter couldn't be used for this work, because most existing datasets have either the tweets or the friend/follower connections between users. For this specific experiment, an emotion network based on the text of the users including the user data was needed, not just the information about who is following whom. For the emotion network, the connections between users were required based on their emotion on a specific issue. So, few recent events and issues were selected to collect tweets with various emotions with search keywords - \#Syria, \#DonaldTrump, \#SchoolShooting, \#Christmas2017, \#NewYear, \#ValentinesDay2018, \#Terrorism, \#olympicgames2018, \#WomensDay, \#Oscars2018.

The dataset was prepared in few steps - (i) collecting random tweets on a keyword; (ii) collecting user information like user ID(unique ID generated by Twitter), user name(the full name of the user), screen name(the name that the user can be addressed by), location(location of the user), number of posts(number of tweets), number of followers(the peo- 
ple who are following the particular user), number of followees(all the people the particular user is following), number of likes; (iii) collecting replies on each tweet; (iv) collecting user information of the repliers; (v) collecting retweets on the tweet; and (vi) collecting user information of each retweeter. These steps were repeated for all search keywords.

Few challenges emerged while collecting data from Twitter that included: (i) Some tweets shared photos and videos and didn't mention much in the text. (ii) Even for tweets which were expressed as text in English, lots of comments were in other languages. (iii) Lots of comments had no text, they just shared photos or videos. (iv) In some cases, lots of replies were posted by persons who tweeted as reply to repliers. Some people replied to each reply on their tweet making the number of replies on their tweets double. (v) Some replies on some tweets were not from another regular user, but from some accounts of news channels or business persons. Those replies were basically advertisements of some news or product. For example, in the replies of \#WomensDay tweets, few advertisement replies were from some news media which work for gender equity, few replies were from business accounts with advertisement of their beauty products, and so on. (vi) Few people had thousands of tweets, but none of them were original. They just retweeted others tweets. (vii) Few replies didn't have any text other than few mentions of some accounts. (viii) Few replies just had the same hashtagged word as the original tweet and nothing else. (ix) Few tweets and replies just stated some facts without expressing any sentiments or emotions. (x) Few replies just had emojis and no text. (xi) Some repliers responded by asking questions which didn’t express any emotion. (xii) Some responses were totally random and out of context.

Both Twitter API and web-page scraping were used for data collection from Twitter. Table 4.1 shows attributes of the tweets and Table 4.2 shows the attributes of a user object that can be extracted using Twitter API. There are few profile images and background images attributes available which were not mentioned in the tables. Due to the rate limit of 


\begin{tabular}{|c|c|c|c|}
\hline created_at & id & id_str & text \\
\hline source truncated & in_reply_to_status_id & in_reply_to_status_id_str & in_reply_to_user_id \\
\hline in_reply_to_user_id_str & in_reply_to_screen_name & user & coordinates \\
\hline place & quoted_status_id_str & is_quote_status & quoted_status \\
\hline retweeted_status & quote_count & reply_count & retweet_count \\
\hline favorite_count & entities & extended_entities & favorited \\
\hline retweeted & possibly_sensitive & filter_level & lang \\
\hline
\end{tabular}

Table 4.1: Tweet Attributes

\begin{tabular}{|c|c|c|c|c|}
\hline id & id_str & name & screen_name & location \\
\hline url & description & derived & protected & verified \\
\hline followers_count & friends_count & listed_count & favourites_count & statuses_count \\
\hline created_at & utc_offset & time_zone & geo_enabled & lang \\
\hline
\end{tabular}

Table 4.2: User Attributes

Twitter API, 15 API calls are allowed in every 15 minutes and this restricted the amount of data collected for the research.

\section{$4.2 \quad$ Pre-processing}

Figure 4.1 shows the work-flow of creating the dataset. First, a crawler was used to find original tweets on a specific topic. The tweets were filtered while collecting tweets and only original tweets (not retweets) were accepted on the topic. The first crawling operation returned unique tweet ID and tweet text which were applied on another crawler to find the number of likes, retweets and time of tweet. Another crawler returned retweeters user IDs. No direct functions exist for extracting replies to a tweet using the Twitter API. Thus, replies to a tweet were collected using a different crawler. The latter crawler took the tweet ID and user ID and checked the texts directed to the user since the time of the tweet. It collected only texts which were true for the parameter 'in_reply_to_status_id'. As this was not using any direct function, collecting replies of a tweet took most of the time. 


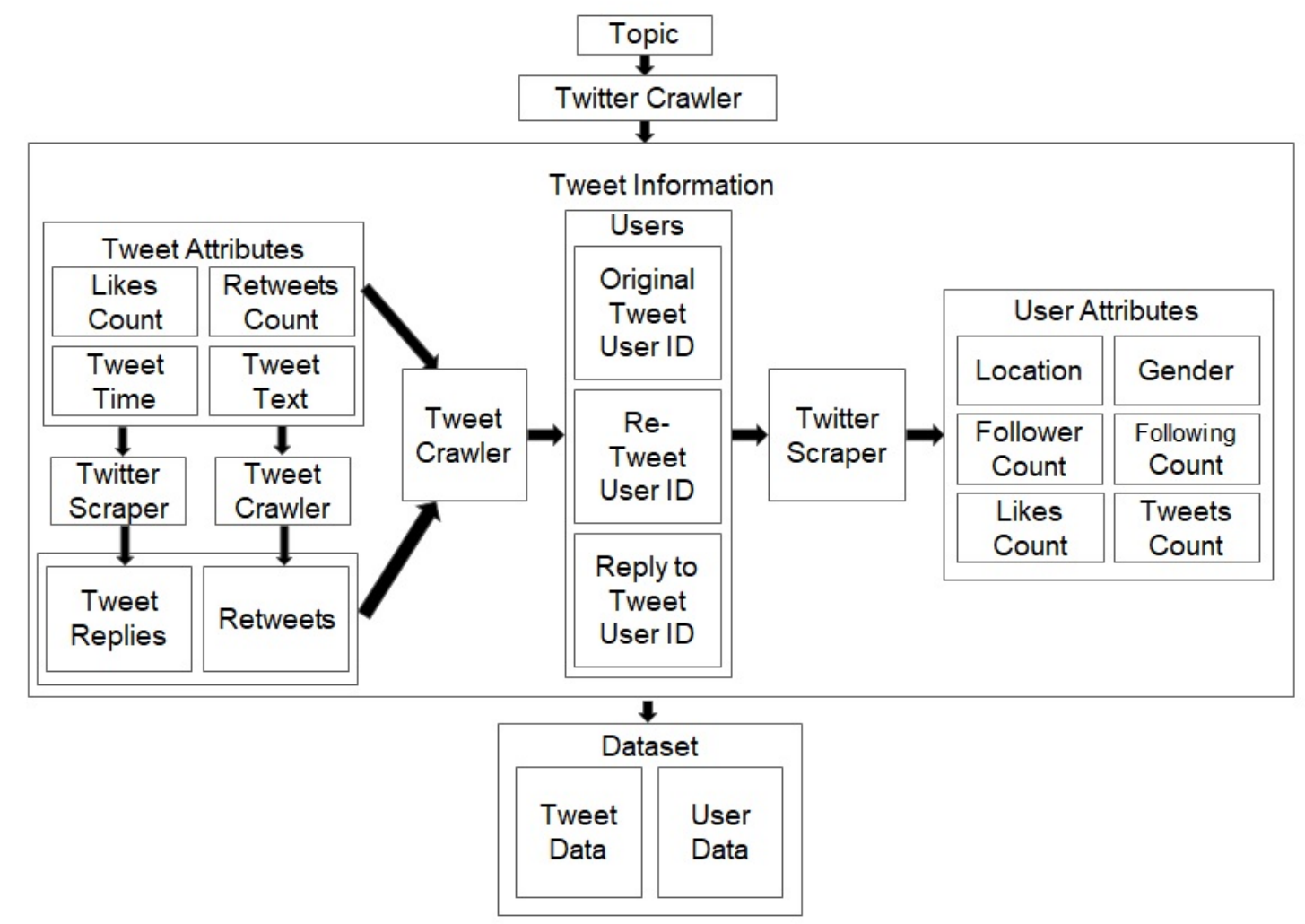

Figure 4.1: Dataset Creation Workflow.

To enhance the speed of collecting replies of tweets, a Web page scraper was used where replies were collected by scraping the tweet page. User information was collected as well for this research. A Web page scraper was used to extract users' data from their Twitter Web page for original tweet users, retweeters and repliers. User attributes collected were the number of tweets, likes, follower, followee and location (when available). In the final dataset, all user data and tweet data were combined. Figure 4.3, Figure 4.4, Figure 4.5 and Figure 4.6 are samples of tweet, reply, retweet and user datasets consecutively.

Data preprocessing included cleaning the collected data and annotating the data according to sentiments and emotions. Tweets and comments had lots of unnecessary symbols and noise. Figure 4.2 shows the steps of preprocessing. The data cleansing process followed these steps: (i) all user mentions (i.e., @alice) were removed from the text and added as a connec- 


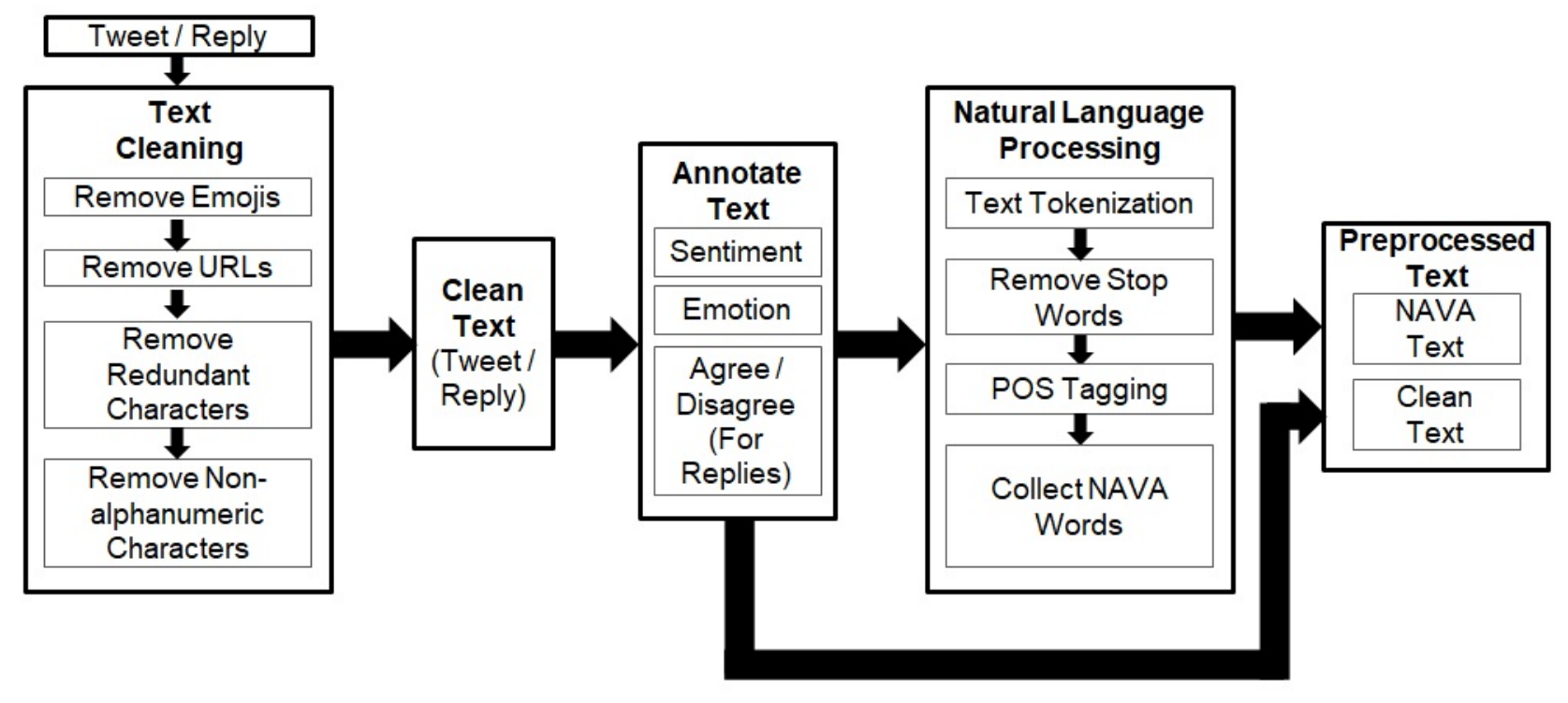

Figure 4.2: Preprocessing Workflow.

\begin{tabular}{|c|c|c|c|c|c|c|c|c|c|}
\hline UserID & TweetII & Retweets & Likes & Replies & TweetTime & Tweet & Topic & Sentiment & Emotion \\
\hline $1173863894^{\prime}$ & $968177563439857664^{\prime}$ & 463 & 768 & 31 & 9:34 AM - 26 Feb 2018 & $\begin{array}{l}\text { Killing of innocent children and women in \#Syria is } \\
\text { strongly condemnable. UN and all other human rights } \\
\text { organizations must play their role to save humanity and }\end{array}$ & \#syria & Negative & Disgust \\
\hline $126384734^{\prime}$ & $968009367168536576^{\prime}$ & 925 & 848 & 66 & 10:26 PM - 25 Feb 2018 & SOUR MEDIA IS FAILING TO REPORT THE FULL TRU & \#syria & Negative & Disgust \\
\hline $1561077758^{\prime}$ & $971929948410793984^{\prime}$ & 1995 & 5988 & 375 & 6:05 PM-8 Mar 2018 & I salute all the conservative women today who fight for or & \#WomensDay & Positive & Joy \\
\hline $17980523^{\prime}$ & $970673331035955200^{\prime}$ & 215 & 515 & 35 & 6:52 AM-5 Mar 2018 & If America hates Tramp, why don't they love the shows the & \#0scars2018 & Negzative & Surprise \\
\hline 19397785' & $970514262832463872^{\prime}$ & 1178 & 15755 & 274 & 8:20 PM-4 Mar 2018 & So happy and proud for you @ JordanPeele! Thanks again & $\# 0$ scars2018 & Positive & Joy \\
\hline $2400907280^{\prime}$ & 971979563528896517 & 63 & 776 & 12 & 9:22 PM-8 Mar 2018 & The sign of a beautiful woman is they always see the beau & \#WomensDay & Positive & Joy \\
\hline 2820265567 & $968005032367988736^{\prime}$ & 128 & 581 & 14 & 10:09 PM - 25 Feb 2018 & 8 What a pathetic world we're living on? What did these po & \#syria & Negative & Disgust \\
\hline $312696314^{\prime}$ & 970969985345400834 & 205 & 193 & 19 & 2:30 AM-6 Mar 2018 & A year before the war began \#Syria had plans to produce & \#syria & Negative & Anger \\
\hline $48038539^{\prime}$ & 969422253757812737 & 110 & 179 & 11 & 8:00 PM-1 Mar 2018 & \#Syria: We know that that defending our country will cost & \#syria & Negative & Sadness \\
\hline $739784130^{\circ}$ & $971865392279777280^{\circ}$ & 8904 & 35307 & 2500 & 1:49 PM- 8 Mar 2018 & $\begin{array}{l}\text { This \#womensday I'd like to thank my mama who's my } \\
\text { best friend; i love u. Who inspires u? \#pressforprogress }\end{array}$ & \#WomensDay & Positive & Joy \\
\hline $25058809676075010^{\circ}$ & $970777015652581376^{\prime}$ & 674 & 661 & 61 & 1:44 PM - 5 Mar 2018 & Heavy bombing in \#EastGhouta. Noor's Arabic teacher ha & \#syria & Negative & Fear \\
\hline
\end{tabular}

Figure 4.3: Sample Tweet Dataset.

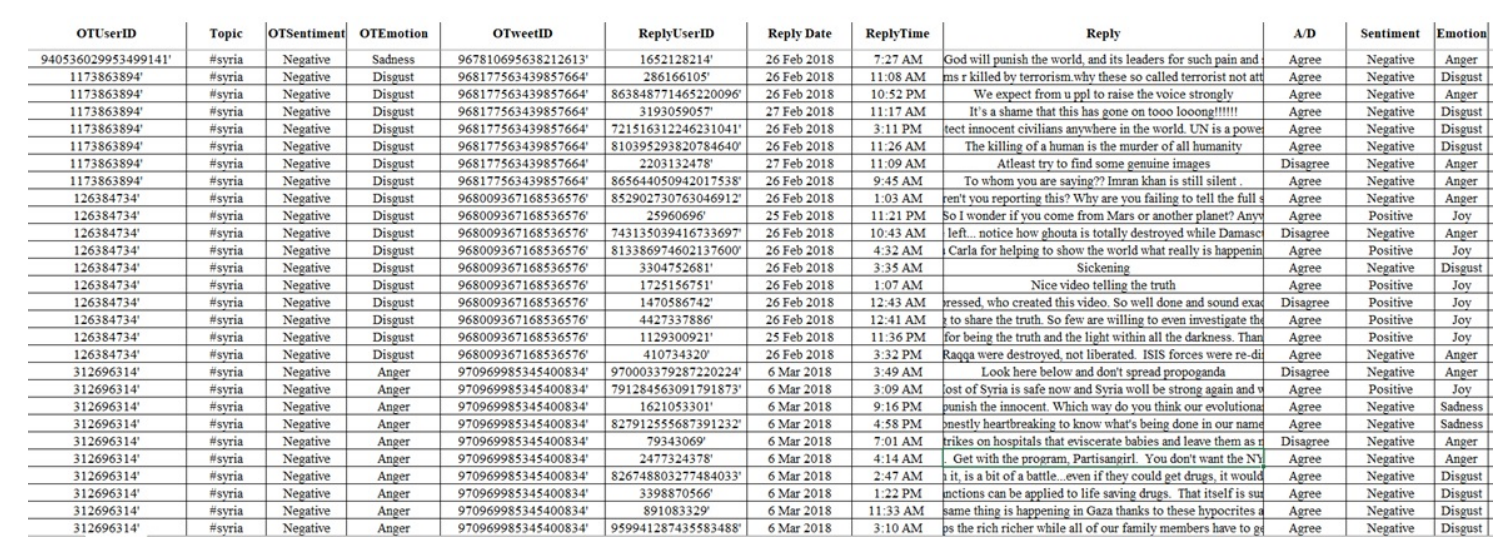

Figure 4.4: Sample Reply Dataset. 


\begin{tabular}{|c|c|c|c|}
\hline 'OTUserID' & 'TweetID' & 'RetweeterID' & Topic \\
\hline $1104070280630272^{\prime}$ & '978652593546252288' & '830964611205718016' & \#SchoolShooting \\
\hline '767104070280630272' & '978652593546252288' & '732078408' & "SchoolShooting \\
\hline '4899575941' & "978718074990944257" & '846190758696673280' & \#SchoolShooting \\
\hline '4899575941' & $978718074990944257^{\prime}$ & $946358671067570176^{\circ}$ & \#SchoolShooting \\
\hline '4899575941' & "978718074990944257" & '2265799817' & \#School Shooting \\
\hline '4899575941' & "978718074990944257" & '792425445929078788' & \#SchoolShooting \\
\hline '4899575941' & "978718074990944257" & '826867021086343169' & \#SchoolShooting \\
\hline $4899575941^{\prime}$ & "978718074990944257" & ${ }^{\circ} 459471568^{\prime}$ & \#SchoolShooting \\
\hline '4899575941' & "978718074990944257" & '919722437616418816" & \#SchoolShooting \\
\hline '4899575941' & '978718074990944257" & '607371255' & "SchoolShooting \\
\hline '4899575941' & "978718074990944257" & '874074353889140736' & \#SchoolShooting \\
\hline '4899575941' & "978718074990944257" & '799605330200186880' & \#SchoolShooting \\
\hline '4899575941' & "978718074990944257" & '558607687' & \#SchoolShooting \\
\hline '4899575941' & "978718074990944257" & $.1607390360^{\prime}$ & \#SchoolShooting \\
\hline '4899575941' & 978718074990944257 & '826124051869528065' & \#SchoolShooting \\
\hline '4899575941' & "978718074990944257" & $256321188^{\prime}$ & \#SchoolShooting \\
\hline '4899575941' & 978718074990944257 & $42480090766^{\prime}$ & "WSchoolShooting \\
\hline $4899575941^{\prime}$ & "978718074990944257" & $' 768166136106057729$ ' & "SchoolShooting \\
\hline '4899575941' & 978718074990944257 & '818290710730379265' & \#SchoolShooting \\
\hline '4899575941' & "978718074990944257" & '856923191926824961' & \#SchoolShooting \\
\hline '4899575941' & 978718074990944257 & $947293112774389760^{\circ}$ & \#SchoolShooting \\
\hline '4899575941' & "978718074990944257" & '821037202968870912' & \#SchoolShooting \\
\hline 91122212005054 & 97879364585 & 78801159865 & \#SchoolShoo \\
\hline '911222120050544645" & "978793645854863360" & '816037443468197892' & \#SchoolShooting \\
\hline
\end{tabular}

Figure 4.5: Sample Retweet Dataset.

\begin{tabular}{c|l|c|c|c|c|} 
UserID & TweetLocation & \#Tweets & \#Followers & \#Likes & \#Following \\
\hline 2820265567 & Hyderabad, India & 1363 & 390000 & 391 & 18 \\
\hline 940536029953499141 & Los Angeles, USA & 7817 & 1159 & 16500 & 665 \\
\hline 1173863894 & Punjab, Pakistan & 6766 & 235000 & 485 & 55 \\
\hline 126384734 & Los Angeles, USA & 9050 & 80900 & 10300 & 387 \\
\hline 312696314 & Syria & 46600 & 71900 & 15100 & 974 \\
\hline 48038539 & Syria & 40200 & 5695 & 1430 & 189 \\
\hline 932954218406617088 & USA & 2412 & 4365 & 3067 & 4991 \\
\hline 925058809676075010 & Syria & 232 & 8590 & 791 & 70 \\
\hline 19397785 & USA & 12400 & 41900000 & 149 & 290 \\
\hline 17980523 & Florida, USA & 112000 & 340000 & 19800 & 8762 \\
\hline 1561077758 & Spartanburg, USA & 11200 & 77300 & 51200 & 38600 \\
\hline 2400907280 & Philippines & 477 & 16300 & 7983 & 409 \\
\hline 739784130 & Mexico & 11500 & 647000 & 19900 & 16800 \\
\hline & & & & &
\end{tabular}

Figure 4.6: Sample User Dataset.

tion between users in the network; (ii) all hashtags (only the \# symbol) were removed; (iii) all emoticons were removed (i.e., :-), :-( etc.); (iv) all multiple occurrences of the same letter were removed (i.e., 'wowwwwwww' became 'wow'); (v) all non-alphanumeric characters (not in A-Z or a-z or 0-9) were removed (i.e., ?, ,, ., ;, ! etc.); (vi) all URLs were removed (i.e., http://a.com). Clean tweets and replies were then annotated.

All tweets and replies were annotated with 'positive', 'negative' or 'neutral' sentiments. For emotion annotations, Ekman's emotion model[38] was used which lists six basic human emotions - 'Anger', 'Disgust', 'Fear', 'Joy', 'Sadness' and 'Surprise'. All tweets and replies were annotated using these six emotions. Replies on tweets were also annotated with 'agreed' or 'disagreed' based on their agreement or difference on the original tweet text. This version of texts was stored as 'Clean Text'. After annotating tweets and replies, Natural Language 


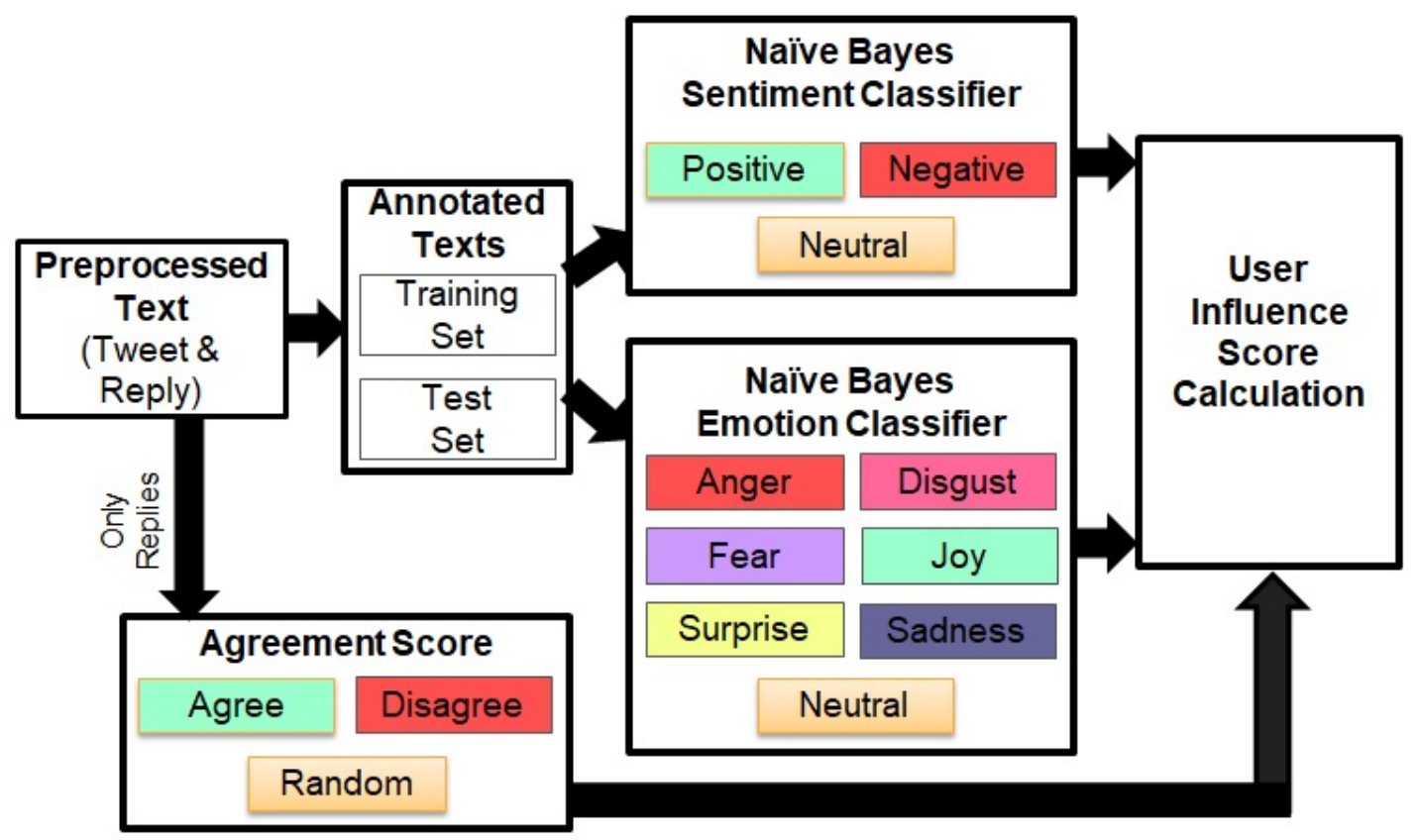

Figure 4.7: Classification \& Recommender System Workflow.

Processing (NLP) was used to process the texts. Words of the sentences were tokenized. Then common English stop words (i.e., am, as, the, etc.) were removed from the text and words were tagged according to their corresponding Parts-of-Speeches(POS) using a POS tagger. Among all words, only Noun, Adjective, Verb and Adverb(NAVA) words were collected as they contribute most in a sentence. These texts were stored as 'NAVA Text' and finally, the preprocessed text were extracted that had two versions of the texts - the cleaned texts and only the NAVA words of each piece of text.

\subsection{Emotion Detection}

In previous studies of emotion detection from text, different methods were used by various researchers. In most recent researches, machine learning was used with both supervised[57, 62] and unsupervised[70, 65] classifiers. Machine learning is a better option for a larger dataset and the training of the classifier is a more generalized approach than creating emotion word dictionaries. Naive Bayes worked better than other machine learning approaches described 
in the literature with sentiment and emotion detection from text. Therefore, Naive Bayes

algorithm was chosen to classify tweets and replies into their corresponding sentiment and emotion classes.

Figure 4.7 shows a work flow of classification of preprocessed text and calculation of influence score to generate recommendation. The preprocessed text (both tweets and replies) were divided into training and test sets for emotion and sentiment classifications. Then, emotion and sentiment were fed into the calculation of "user influence score" which also used the agreement score for replies (i.e., if the reply agreed to the original tweet or not). The influence score used all these values along with the number of tweets, retweets, follower and followees to generate the final influence score. The recommender system took the score and generated a list of users as a recommendation who shared similar emotion and sentiment on a specific topic.

The Naive Bayes algorithm was applied with 3-fold, 5-fold and 10-fold cross validation on the preprocessed text (tweets and replies). The feature set used for Naive Bayes were NAVA words for 'NAVA Text' and all the words for 'Clean Text' from each tweet and reply. The classifier was applied on the same data set twice - (i) for classifying them according to their sentiments and (ii) for classifying them according to their corresponding emotions.

\subsection{Influential Users Detection}

In literature, researchers applied only the numeric scores like number of followers, followees to detect the influential users from a network. But, in this research, few other factors were considered for the calculations. One person can influence another person in both positive and negative ways. Normally, when someone likes or retweets a tweet from another person, 


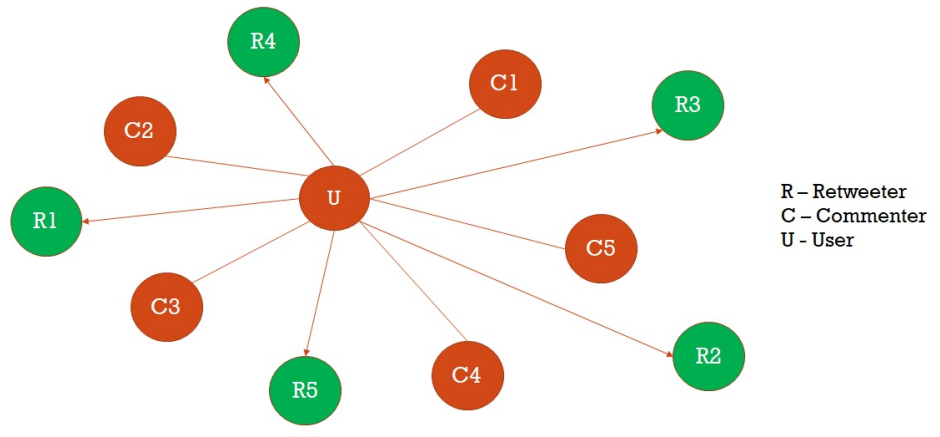

Figure 4.8: Connections between a Tweeter and the Retweeters and Repliers.

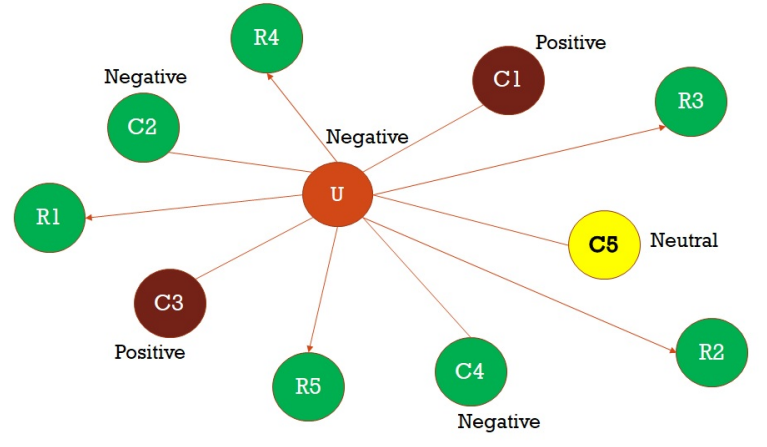

Figure 4.9: Sentiments of Nodes.

he/she agrees to that tweet. But a person can reply on another person's tweet and express either his/her support or disagreement to that tweet. This was addressed while calculating the influence score based on all the factors - (i) Number of retweets, (ii) Number of likes, (iii) Number of comments that agree to the tweet or show similar sentiment/emotion, (iv) Number of comments that disagree to the tweet or show opposite sentiment/emotion, and (v) Number of people who follow the person.

Figure 4.8 represents a sample of connections between the user of the tweet (U) and the retweeters $(\mathrm{R})$ and repliers $(\mathrm{C})$ of that tweet. The retweeters are influenced by the tweeter and are shown in green nodes; $\mathrm{U}$ influences $\mathrm{R}$ nodes. In Figure 4.9, the sentiments of the replies are shown as positive, negative or neutral. It shows people having similar sentiments to the user. Even if some nodes are expressing same sentiments on a certain issue, they still can have different emotions. For example, on some horrific incident, one person can express 


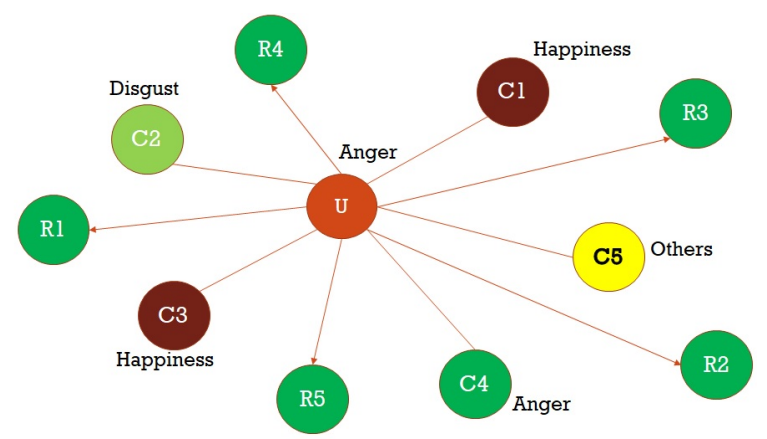

Figure 4.10: Emotions of Nodes.

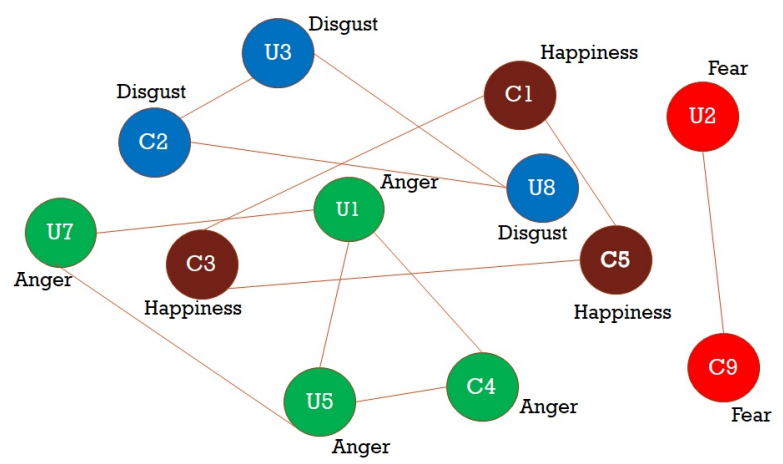

Figure 4.11: Suggestions of Connections between Similar Emotion Nodes.

anger whereas another person can express disgust, but anger and disgust both are negative sentiments. Figure 4.10 represents emotions of the nodes. Figure 4.11 represents possible suggestions for users to let them know people who are not directly connected, but have the same emotion regarding any social, national or international issue.

Due to the variety of parameters and metrics of Twitter data, there is no one standard equation to measure influential users in Twitter. Various researchers used different measurements based on different perspectives and statements of their research problems.

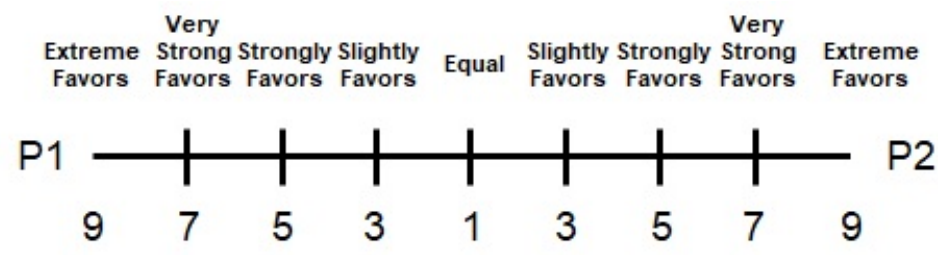

Figure 4.12: Pairwise Comparison Scale in AHP. 
Existing measurements were modified according to the problem statement and two types of influence scores (IS) of a user were measured using the following values. $W$ values are the weights assigned to each parameter. These weights were calculated using Analytical Hierarchy Process(AHP)[114]. Weight calculation was done using the following steps. (i) Parameters were divided into two groups: (a) user-based parameters (Number of tweets, followers, followees and likes), (b) tweet-based parameters (number of retweets, replies, likes, agreement, sentiment and emotion scores). (ii) For each group of parameters, a pairwise comparison was done between every combination of two parameters using the scale shown in Figure 4.12. $\mathrm{P} 1$ and $\mathrm{P} 2$ are any two parameters and values from 1 to 9 are measurements of their comparison. (iii) Two different comparison matrix for two groups were created. (iv) After creating the Eigen vectors for both matrices, normalized Eigen vectors were used as priority matrices for weight vectors. A general influence score $\left(I S_{G}\right)$ for each user was calculated based on user-based parameters. Another topic specific influence score $\left(I S_{T}\right)$ was computed by merging user-based and tweet-based parameters.

User-based Parameters:

$T S_{U}($ Tweet_Score $)=W_{T} * \#$ Tweets

$F S_{U}($ Follower_Score $)=W_{F} * \#$ Followers

$O S_{U}($ Followee_Score $)=W_{O} * \#$ Followees

$L S_{U}($ Like_Score $)=W_{L} * \#$ Likes

Tweet-based Parameters:

$R S_{T}($ Retweet_Score $)=W_{R} * \#$ Retweets

$C S_{T}($ Comment_Score $)=W_{C} * \#$ Comments

$P S_{T}($ Like_Score $)=W_{P} * \#$ Likes

$A S_{T}($ Agreement_Score $)=W_{A} *$ Agreement_Value

$S S_{T}($ Sentiment_Score $)=W_{S} *$ Sentiment_Value 
$E S_{T}($ Emotion_Score $)=W_{E} *$ Emotion_Value

$$
\begin{aligned}
& \text { Agreement_Value }=\left\{\begin{array}{rr}
1 & \text { if reply agrees with tweet } \\
-1 & \text { if reply disagrees with tweet }
\end{array}\right. \\
& \text { Sentiment_Value }=\left\{\begin{array}{rr}
1 & \text { similar sentiment } \\
-1 & \text { different sentiment }
\end{array}\right. \\
& \text { Emotion_Value }=\left\{\begin{array}{rr}
1 & \text { similar emotion } \\
-1 & \text { different emotion }
\end{array}\right.
\end{aligned}
$$

The two Influence_Scores (IS) are,

$$
\begin{gathered}
I S_{U}=T S_{U}+F S_{U}+O S_{U}+L S_{U} \\
I S_{T}=I S_{U}+R S_{T}+C S_{T}+P S_{T}+A S_{T}+S S_{T}+E S_{T}
\end{gathered}
$$

To normalize IS values, the values were converted into percentage using the following formula, where MIN and MAX are the minimum and maximum values of IS (Influence Score) among all users in the dataset.

$$
\frac{I S-M I N}{M A X-M I N} * 100
$$

\subsection{Recommendation}

The recommender system provided two different types of recommendation on both emotion and sentiment. (i) General Recommendation: A common recommendation based on a specific topic which recommended top ten user IDs on each sentiment and emotion. (ii) Personalized Recommendation: For each user, a recommendation which recommended top ten 


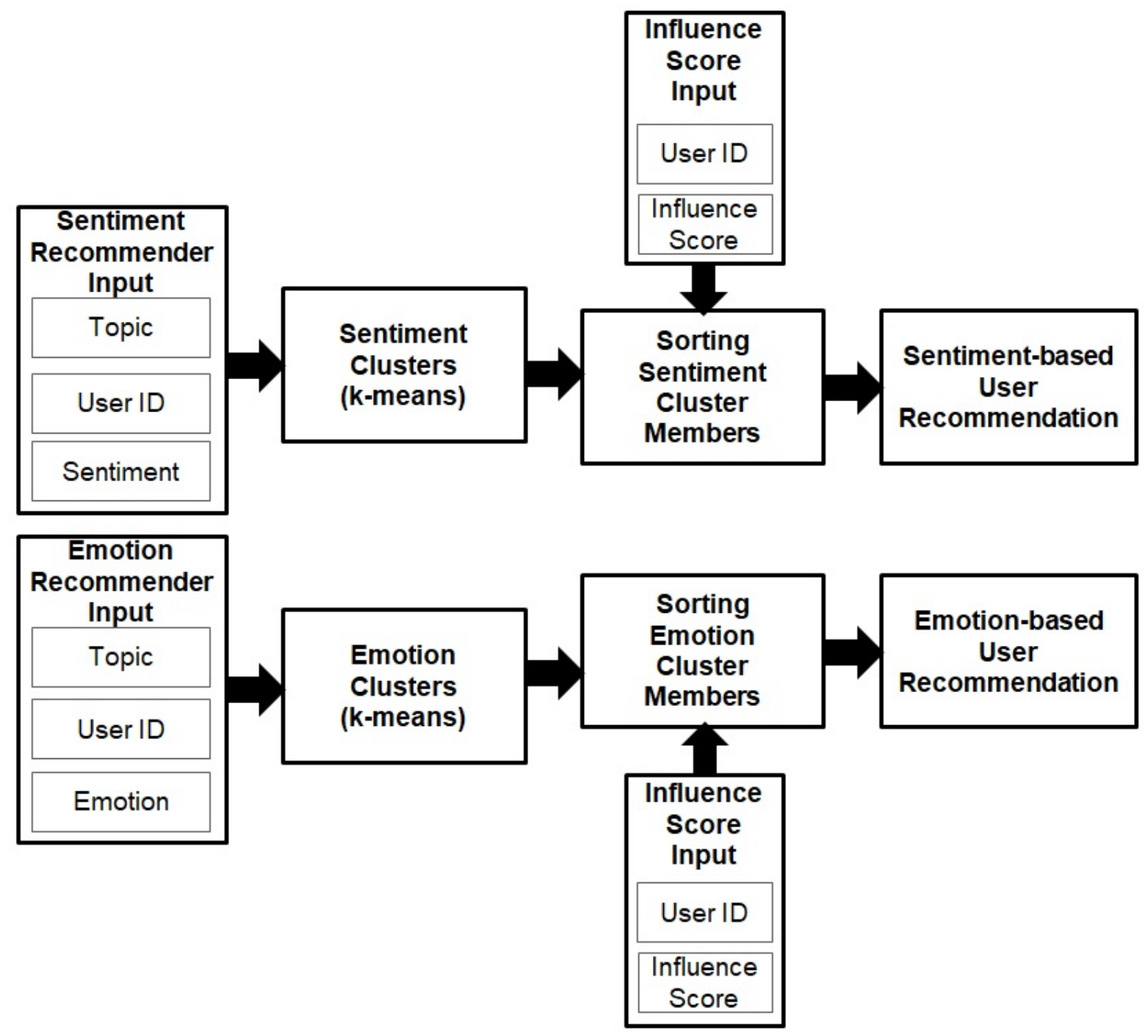

Figure 4.13: Recommender System Workflow.

user IDs who shared similar emotion and sentiment on a specific topic. The general recommender found users who interacted (tweeted, replied, retweeted) on a specific topic. Each of those users expressed some emotion and sentiment regarding that particular topic. Therefore, k-means clustering was applied individually based on sentiment and emotion to create individual clusters for each emotion and sentiment. As each user had a corresponding emotion and a sentiment regarding a specific topic, for each topic, k-means clustering method created clusters of users according to their emotion and sentiment towards that topic. Since k-means clustering needs the number of clusters prior to any calculation, the method worked 
well for this procedure. The number of clusters of sentiment and emotion recommender were equal to the number of sentiments and emotions considered. For sentiment, three clusters (positive, negative and neutral) were formed. Each user was assigned to a cluster according to his/her sentiment on the topic. Then, all users of each cluster were sorted according to their influence score and the first ten users with highest influence scores were recommended. The same process was repeated for emotion as well. Seven clusters for emotion were formed, namely anger, disgust, fear, joy, sadness, surprise and neutral. Figure 4.13 shows the workflow of the recommender system. For the personalized recommendation, for each user of a cluster, a highest influence score ranked list of users from the same cluster was generated as a customized recommendation to that user to suggest him/her about other people sharing similar emotion or sentiment on a topic.

The framework for the data collection, annotation, classification and recommendation used few existing ideas and parameters from literature and combined them with some new framework units and calculation parameters. The workflow diagrams of each unit showed the process in detail with all the considered features. The equations used included parameters with proper definition and reasoning behind the calculations for experimental setup. 


\section{Chapter 5}

\section{Experimental Results}

The experiments and results discussed in this chapter were the outcomes of the methodology and procedures explained in chapter 5 . As the dataset and methodology of chapter 4 differ from the dataset and procedure of chapter 3 , chapter 5 has nothing in common with the experimental results of chapter 3 . The experiments for this research were conducted in multiple steps. The data collection process with sample results were described in Chapter 4 . In this chapter, all the preprocessing done on the collected data are mentioned with classification accuracy scores, sample influence scores, sample general and personalized recommendations for both sentiments and emotions.

\subsection{Emotion and Sentiment Classification}

After collecting the data, the texts were cleaned and preprocessed. Then Naive Bayes classifier was applied to classify the text according to corresponding sentiment and emotion classes using 3-fold, 5-fold and 10-fold cross validation. The sentiment and emotion classifiers were applied on the cleaned text and on only the NAVA words individually to measure the classification accuracy. Table 5.1 shows accuracy results in percentage for sentiment and emotion classification for both full text and text with only NAVA words. The table 
also shows the accuracy of emotion classification accuracy of Naive Bayes on the benchmark ISEAR dataset[83]. The emotion classes in the new dataset and the ISEAR dataset had five emotions in common. Instead of 'surprise', ISEAR included 'shame' and 'guilt'. The accuracy of emotion classification of ISEAR dataset were included to show variation in results of the same classifier with two different datasets with slightly varying emotions.

According to the results, using full text for classification increased accuracy by at least $5 \%$ than using only NAVA words. Also, as the number of folds were increased for the k-fold cross validation, classification accuracy increased gradually. Another interesting observation was the differences between accuracies in sentiment and emotion classifications. For sentiment classification, 3 classes (positive, negative, neutral) existed whereas there were 7 classes for emotion (anger, disgust, fear, joy, sadness, surprise and neutral). As the number of classes increased for emotion classifier, the accuracy level decreased a little bit compared to the sentiment classifier. As expected from a text based classifier, the accuracy levels were not very high. The reason behind this was the structure of the data. For Twitter data (tweets

and replies), finding proper and grammatically correct sentences with appropriate parts-ofspeeches was difficult. On the other hand, for the benchmark ISEAR dataset, as the texts were properly formed meaningful sentences, the accuracy level of classification was higher than the Twitter data. The distribution of emotions in the dataset was another reason for the lower accuracy. As the tweets and replies were collected randomly, all the emotions were not very well-distributed in the dataset which resulted lower classification accuracy.

\section{$5.2 \quad$ Influence Score}

The next step was measuring the influence score for users. AHP was used to calculate the weight vector. The comparison matrices is shown in Figure 5.1. The priority matrices were 


\begin{tabular}{|c|c|c|c|c|c|c|}
\hline $\begin{array}{c}\mathrm{k}- \\
\text { fold }\end{array}$ & $\begin{array}{c}\text { Full Text } \\
\text { Sentiment }\end{array}$ & $\begin{array}{c}\text { NAVA } \\
\text { Sentiment }\end{array}$ & $\begin{array}{c}\text { Full Text } \\
\text { Emotion }\end{array}$ & $\begin{array}{c}\text { NAVA } \\
\text { Emotion }\end{array}$ & $\begin{array}{c}\text { ISEAR[83] } \\
\text { Dataset } \\
\text { Full Text } \\
\text { Emotion }\end{array}$ & $\begin{array}{c}\text { ISEAR[83] } \\
\text { Dataset } \\
\text { NAVA } \\
\text { Emotion }\end{array}$ \\
\hline \begin{tabular}{c}
\hline- \\
fold
\end{tabular} & 62.69 & 55.96 & 44.37 & 40.45 & 52.34 & 53.37 \\
\hline $\begin{array}{c}5- \\
\text { fold }\end{array}$ & 63.71 & 58.33 & 46.32 & 41.29 & 54.01 & 54.82 \\
\hline $\begin{array}{c}10- \\
\text { fold }\end{array}$ & 66.86 & 61.15 & 47.34 & 43.24 & 55.11 & 56.51 \\
\hline
\end{tabular}

Table 5.1: Sentiment and Emotion Classification Accuracy (in percentage - \%)

\begin{tabular}{|c|c|c|c|c|}
\hline & TS $_{U}$ & FS $_{U}$ & OS $_{U}$ & LS $_{U}$ \\
\hline TS $_{U}$ & 1 & $1 / 5$ & 7 & 7 \\
\hline FS $_{U}$ & 5 & 1 & 7 & 7 \\
\hline OS $_{U}$ & $1 / 7$ & $1 / 7$ & 1 & 1 \\
\hline LS $_{U}$ & $1 / 7$ & $1 / 7$ & 1 & 1 \\
\hline
\end{tabular}

\begin{tabular}{|c|c|c|c|c|c|c|}
\hline & RS $_{\mathrm{T}}$ & $\mathrm{CS}_{\mathrm{T}}$ & $\mathrm{PS}_{\mathrm{T}}$ & $\mathrm{AS}_{\mathrm{T}}$ & $\mathrm{SS}_{\mathrm{T}}$ & $\mathrm{ES}_{\mathrm{T}}$ \\
\hline $\mathrm{RS}_{\mathrm{T}}$ & 1 & 7 & 3 & $1 / 3$ & 7 & 7 \\
\hline $\mathrm{CS}_{\mathrm{T}}$ & $1 / 7$ & 1 & $1 / 7$ & $1 / 7$ & 1 & 1 \\
\hline $\mathrm{PS}_{\mathrm{T}}$ & $1 / 3$ & 7 & 1 & $1 / 3$ & 7 & 7 \\
\hline $\mathrm{AS}_{\mathrm{T}}$ & 3 & 7 & 3 & 1 & 7 & 7 \\
\hline $\mathrm{SS}_{\mathrm{T}}$ & $1 / 7$ & 1 & $1 / 7$ & $1 / 7$ & 1 & 3 \\
\hline ES $_{\mathrm{T}}$ & $1 / 7$ & 1 & $1 / 7$ & $1 / 7$ & $1 / 3$ & 1 \\
\hline
\end{tabular}

Figure 5.1: Comparison Matrices for AHP.

calculated by eigen vectors and normalization. Finally, the weight vector had the following weight values -

$W_{T}=0.292, W_{F}=0.586, W_{O}=0.061, W_{L}=0.061, W_{R}=0.272, W_{C}=0.040, W_{P}=$ $0.204, W_{A}=0.395, W_{S}=0.053$, and $W_{E}=0.035$.

The agreement score represented if the reply to a tweet agreed or disagreed to the tweet. To apply the influence score formula on the whole dataset (both tweets and replies), 1 (agreement) was assigned as the Agreement_Value for original tweets. This inclusion was logical because the user who tweeted something obviously agreed to his/her own tweet. After the calculation the influence score was normalized between 0 to 100 since the influence scores of users varied from 10 to millions because of the number of tweets, followers etc. Figure 5.2 shows the user IDs with their original and normalized influence scores in a descending 


\begin{tabular}{|c|c|c|}
\hline UserID & IS & Normalized IS \\
\hline $19397785^{\prime}$ & 24557047.58 & 99.99999991 \\
\hline $739784130^{\prime}$ & 384738.7 & 1.566705736 \\
\hline $17980523^{\prime}$ & 233686.282 & 0.951597466 \\
\hline $2820265567^{\prime}$ & 228962.945 & 0.932363324 \\
\hline '77338274' & 219298.639 & 0.893008809 \\
\hline '749038067747590144' & 196021.573 & 0.798221079 \\
\hline '149296998' & 182857.669 & 0.744615673 \\
\hline $1173863894^{\prime}$ & 160579.121 & 0.65389406 \\
\hline '251009806' & 115090.954 & 0.46865937 \\
\hline '1713417312' & 107508.749 & 0.437783486 \\
\hline '17235735' & 107062.369 & 0.435965759 \\
\hline '2258357868' & 105952.716 & 0.431447084 \\
\hline '880929493' & 105707.38 & 0.430448039 \\
\hline '253340432' & 104023.739 & 0.423591999 \\
\hline '839099917121511425' & 103879.948 & 0.42300646 \\
\hline '503794853' & 102850.474 & 0.418814286 \\
\hline '2318083470' & 101315.387 & 0.41256318 \\
\hline '3357427702' & 98647.878 & 0.40170068 \\
\hline '2876041031' & 98647.878 & 0.40170068 \\
\hline '805345210557493248' & 97451.093 & 0.396827191 \\
\hline '348780432' & 96743.078 & 0.393944047 \\
\hline '348780432' & 96743.078 & 0.393944047 \\
\hline '624827127' & 92965.685 & 0.378561932 \\
\hline
\end{tabular}

Figure 5.2: Users and their Influence Scores.

order. An interesting observation from the influence scores is that the difference between the highest score and all the other scores is very high. As Twitter does not enforce the bidirectional follow rule like other social networks, few people (i.e. actors, politicians, journalists, etc.) have sometimes 1000 times more followers than a regular user. That difference still existed after normalizing the scores. They were not considered as outliers because there were multiple users with huge amount of followers and this also represented the uniqueness of Twitter over other social networks.

\subsection{Recommendations}

The influence scores were then used for the recommender system. The general recommender provided top 10 most influential users for each sentiment and emotion. Figure 5.3 and 5.4 


\begin{tabular}{c|c|c} 
UserID & IS & Sentiment \\
\hline $19397785 '$ & 100 & Positive \\
\hline $407474647 '$ & 0.550642859 & Positive \\
\hline '601448080' & 0.539985025 & Positive \\
\hline '601448080' & 0.539985025 & Positive \\
\hline '601448080' & 0.539985025 & Positive \\
\hline $20831692^{\prime}$ & 0.46468827 & Positive \\
\hline $413007007 '$ & 0.314848707 & Positive \\
\hline $90895234 '$ & 0.212021002 & Positive \\
\hline $150422835 '$ & 0.19237308 & Positive \\
\hline$' 3061826623 '$ & 0.15292931 & Positive \\
\hline $17980523 '$ & 1.146551095 & Negative \\
\hline '29703494' & 0.154778385 & Negative \\
\hline $477918460 '$ & 0.083265675 & Negative \\
\hline $368586936 '$ & 0.075857442 & Negative \\
\hline $54158866 '$ & 0.022759141 & Negative \\
\hline$' 3740444776 '$ & 0.014704328 & Negative \\
\hline '3740444776' & 0.014704328 & Negative \\
\hline $862840407159447552 '$ & 0.006537378 & Negative \\
\hline $811007544080662532 '$ & 0.003154166 & Negative \\
\hline$' 891708601093562369 '$ & 0.001586626 & Negative \\
\hline $938907973279154177 '$ & 0.215082117 & Neutral \\
\hline$' 3555274281 '$ & 0.05117049 & Neutral \\
\hline
\end{tabular}

Figure 5.3: General Recommendation according to Sentiment.

show the general sentiment and emotion recommendation on \#Oscars2018. Figure 5.5 shows personalized recommendation for one user. The file name shows user ID for whom the recommendation was issued.

\subsection{Networks between Users}

Figure 5.6 shows the retweet network for tweets on \#Oscars2018. The little circles represent users. Larger circles represent clusters of users who retweeted a tweet of the user who is the center of the cluster. The bigger the circle is, the more retweets that tweet has. This is equivalent to the influence of the user. Few tweets were retweeted later by the author of the tweet. This created few loops in the network. Figure 5.7 shows the retweet network for \#Oscars2018. Figure 5.8 shows the reply network for \#Oscars2018 without considering 


\begin{tabular}{|c|c|c|}
\hline UserID & IS & Emotion \\
\hline '891708601093562369' & 0.001586626 & Anger \\
\hline $54158866^{\prime}$ & 0.022759141 & Disgust \\
\hline $862840407159447552^{\prime}$ & 0.006537378 & Disgust \\
\hline $811007544080662532^{\prime}$ & 0.003154166 & Disgust \\
\hline $814488110306381825^{\prime}$ & 0.000212345 & Disgust \\
\hline '29703494' & 0.154778385 & Fear \\
\hline $19397785^{\prime}$ & 100 & Joy \\
\hline $407474647^{\prime}$ & 0.550642859 & Joy \\
\hline $20831692^{\prime}$ & 0.46468827 & Joy \\
\hline $413007007^{\prime}$ & 0.314848707 & Joy \\
\hline $90895234^{\prime}$ & 0.212021002 & Joy \\
\hline $150422835^{\prime}$ & 0.19237308 & Joy \\
\hline '820232246904950784' & 0.094951834 & Joy \\
\hline $89931829^{\prime}$ & 0.043022627 & Joy \\
\hline $750441536848994304^{\prime}$ & 0.025071083 & Joy \\
\hline $279785200^{\prime}$ & 0.021029361 & Joy \\
\hline '3740444776' & 0.014704328 & Sadness \\
\hline '3740444776' & 0.014704328 & Sadness \\
\hline $17980523^{\prime}$ & 1.146551095 & Surprise \\
\hline '601448080' & 0.539985025 & Surprise \\
\hline '601448080' & 0.539985025 & Surprise \\
\hline '601448080' & 0.539985025 & Surprise \\
\hline '3061826623' & 0.15292931 & Surprise \\
\hline $477918460^{\prime}$ & 0.083265675 & Surprise \\
\hline $368586936^{\prime}$ & 0.075857442 & Surprise \\
\hline '3740444776' & 0.014704328 & Surprise \\
\hline '938907973279154177' & 0.215082117 & Neutral \\
\hline '3555274281' & 0.05117049 & Neutral \\
\hline '882654015284051969' & 0.007735102 & Neutral \\
\hline
\end{tabular}

Figure 5.4: General Recommendation according to Emotion.

\begin{tabular}{c|c|}
\multicolumn{2}{|c}{$279785200^{\prime}$ Rec.csv } \\
\hline UserID & IS \\
\hline $19397785 '$ & 100 \\
\hline$' 601448080^{\prime}$ & 0.539985025 \\
\hline$' 601448080^{\prime}$ & 0.539985025 \\
\hline $413007007 '$ & 0.314848707 \\
\hline $150422835 '$ & 0.19237308 \\
\hline '820232246904950784' & 0.094951834 \\
\hline $750441536848994304 '$ & 0.025071083 \\
\hline '3296075343' & 0.017386085 \\
\hline $831982169253937152^{\prime}$ & 0.016925605 \\
\hline $958527409019432960 '$ & 0.012621434 \\
\hline
\end{tabular}

Figure 5.5: Personalized Recommendation. 


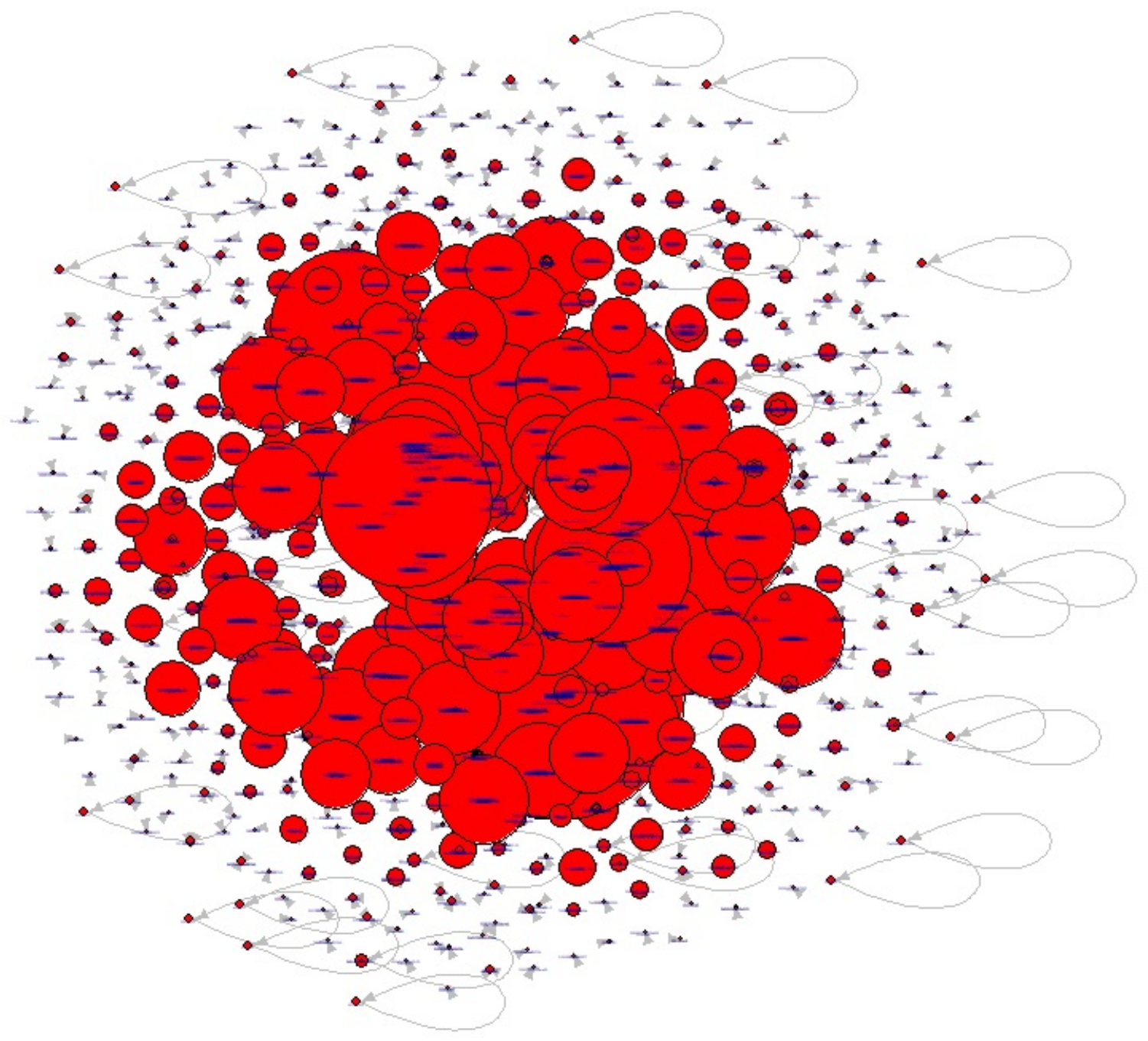

Figure 5.6: Retweet Network based on Degree for \#Oscars2018.

emotions or sentiments. Here, a connection from one node to another means the source node replied to a tweet of the destination node. Figure 5.9 and 5.10 show the reply network connections for \#Oscars2018. They are color coded according to their sentiments and emotions. For the sentiment network, yellow nodes represent positive sentiment, blue nodes represent negative sentiment and green nodes represent neutral sentiment. In the emotion network, dark blue represents joy, pink represents anger, blue represents disgust, orange represents fear, red represents surprise and yellow represents neutral emotion. This shows how most of the connected users show similar sentiment and emotion on a topic. 


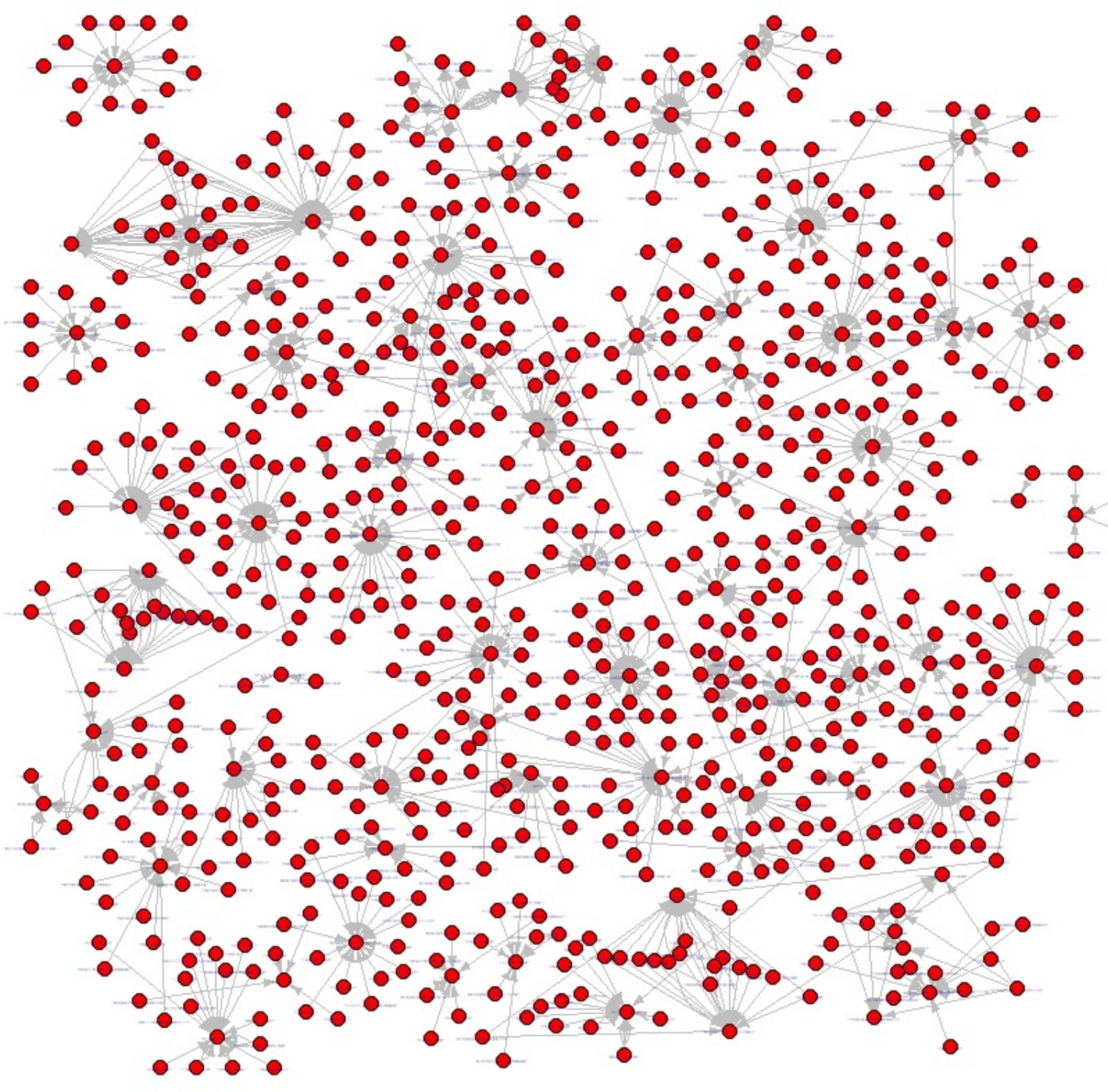

Figure 5.7: Retweet Network for \#Oscars2018. 


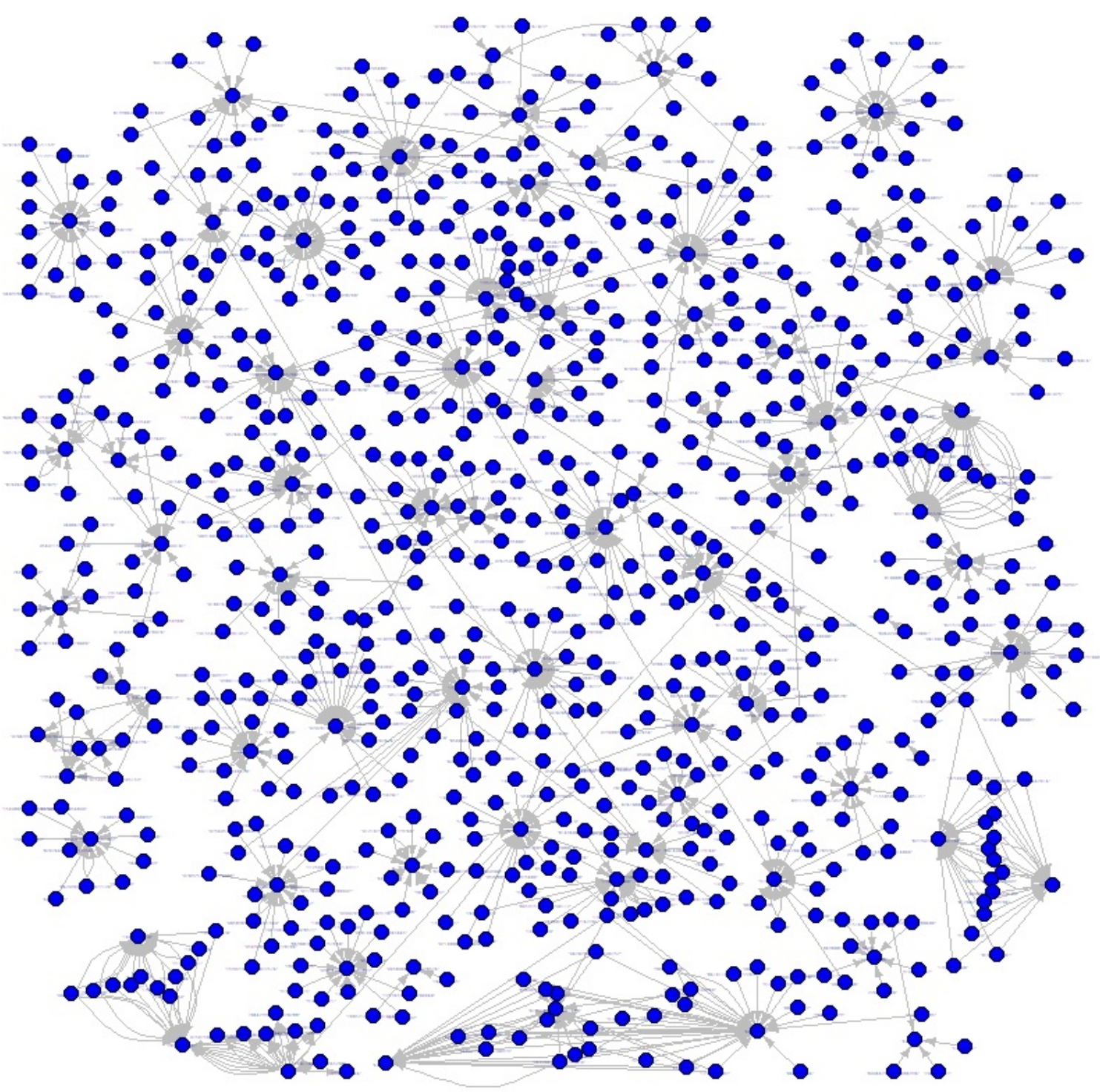

Figure 5.8: Reply Network for \#Oscars2018. 


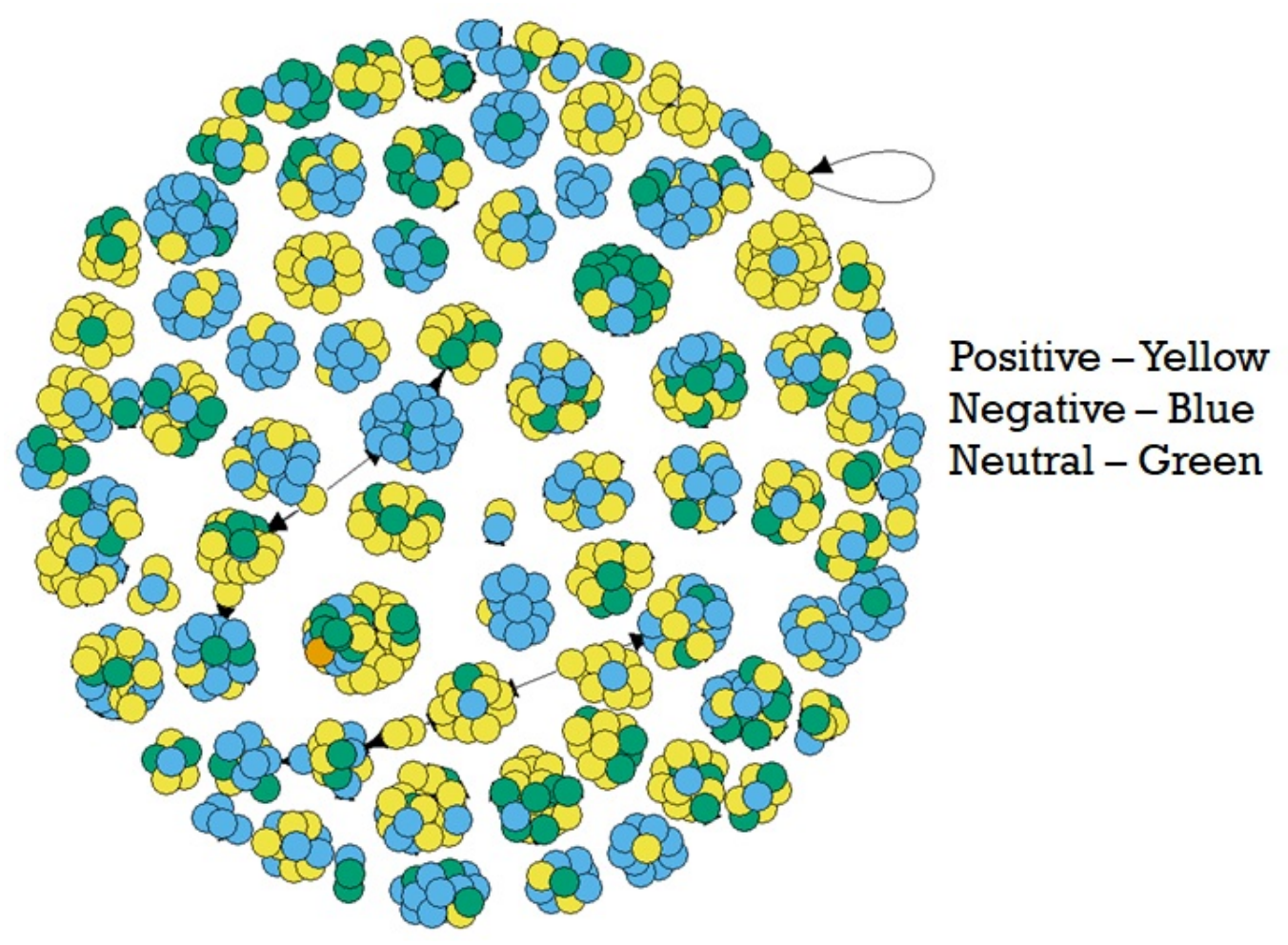

Figure 5.9: Tweet Sentiment Network for \#Oscars2018.

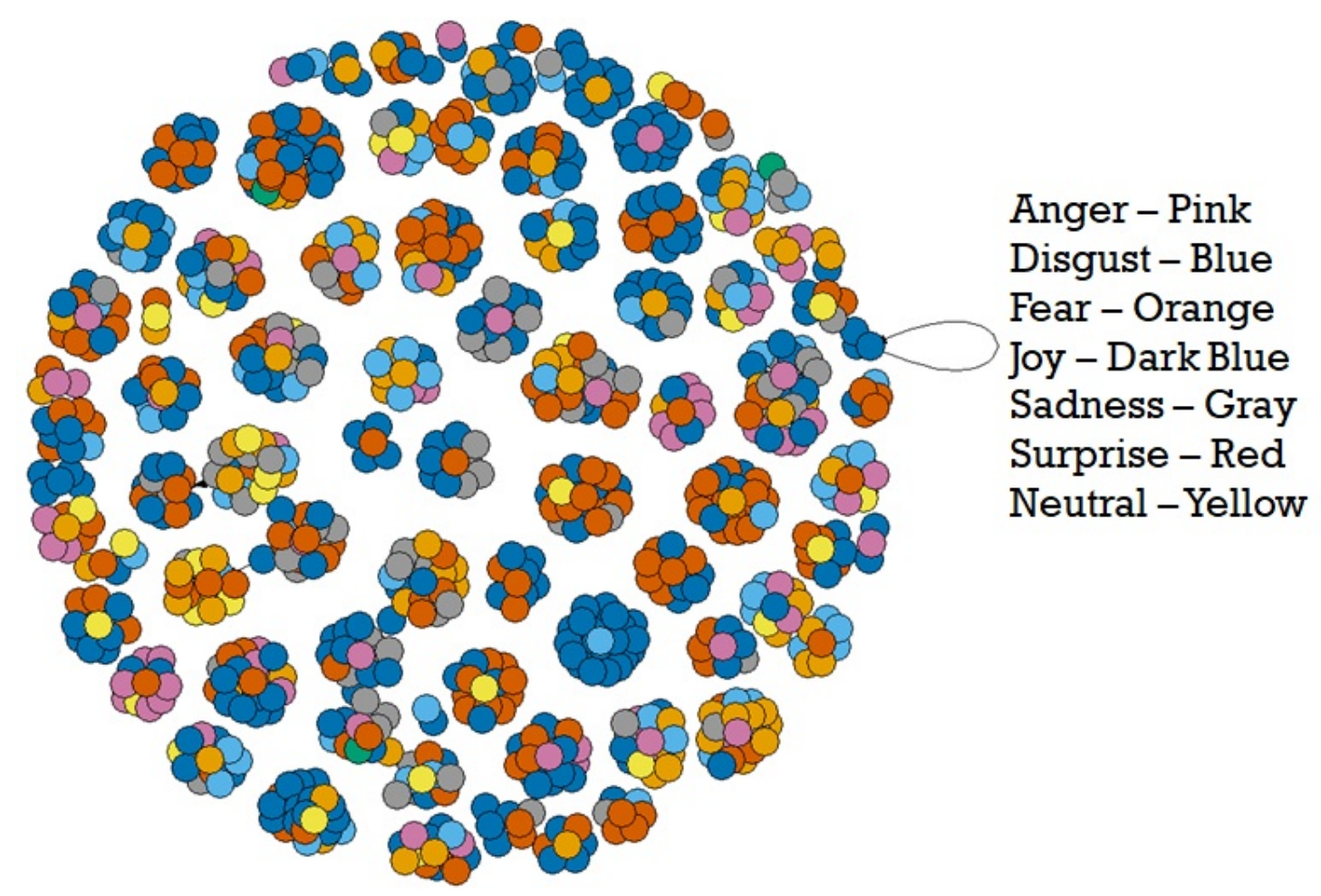

Figure 5.10: Tweet Emotion Network for \#Oscars2018. 
The experimental results mentioned in this chapter lead us to some interesting realizations. The randomness of Twitter data showed it's affect on most of the scores and results to further prove the claim of the previous researchers, that "Twitter is different than the other social networks in terms of all measurable characteristics and therefore do not follow any rules that are applicable for other social networks for most scenarios." 


\section{Chapter 6}

\section{Conclusion and Future Works}

Humans themselves intentionally or unintentionally perform emotion recognition every time they interact with others. Hints may be picked up to classify emotions based on facial expression, speech, and even text. There have been a lot of research works done to categorize emotions and sentiments by considering these information sources. In this thesis, the focus was on reviewing research works performed on categorizing emotions and sentiments based on text and analyzing them. Emotion and sentiment based parameters were used with the other user-based and tweet-based parameters to generate recommendations for users based on emotion and sentiment network.

\subsection{Conclusion}

In this thesis, some recent works on this topic and some possible areas where improvements are possible and needed were covered. Existing works of different researchers have used novel approaches or hybrids of existing approaches to address text based emotion and sentiment detection. They have used only one word or multiple words from the given sentences, they have applied lexicons, implemented machine learning methods, created new datasets for various domains, described emotions and sentiments with diverse models and tried some unique 
ideas. Many different methodologies, such as, SVM, Hybrid systems, Ensemble systems have been used for categorizing emotions and sentiments. Hybrid systems seemed to perform better than systems using just one source of information. Ensemble systems also seemed to perform better than individual classifier. There is a lot of overlap in the methodologies and features used in the research on this topic. Few existing works were summarized to give an idea on the research done on this topic, databases used, features extracted, methodologies used, and the results reported by various researchers.

But despite so many existing works described in the literature, the accuracy of emotion detection from text when the emotion is implicit is not good. Detecting sentiments from texts have better accuracy than detecting emotions due to the fact that sentiments can vary within only three categories- positive, negative or neutral. Detecting sarcasms correctly still needs huge contributions. Classifying emotions according to their intensities is a comparatively new topic. Detecting multiple emotions and sentiments from the same text; realizing the cause behind an emotion or sentiments; detecting the personality of a person; relating emotion and sentiment with different social and individual parameters; detecting the mood of an individual or public in general; predicting the action of a person based on his/her emotion or sentiment - these can be some possible issues to focus on in future. Through the literature review, a better understanding of the research done on this topic, databases used, features extracted, methodologies used, and the results reported by various researchers can be achieved.

The analysis done in this thesis on different emotion and sentiment showed some interesting human characteristics. For all six emotions analyzed, each of them can be characterized by multiple dimensions. For example, 'surprise' is an emotion which can express both 'positive' and 'negative' sentiments. Someone can be surprised by how stupid or disgusting some people are, which is the 'negative' kind of surprise. On the other hand, someone can be 
surprised by how beautiful nature is, which is the 'positive' type of surprise. This occurred in the dataset several times. Sometimes, some repliers' emotion about a tweet was 'surprise' even if they disagreed to the tweet because they were surprised about the stupidity of the tweet. For some tweets, few comments expressed opposite sentiment even though the repliers agreed to the tweet. For example, a tweet from a Syrian who tweeted about their sufferings had most of the comments agreeing to the content. Few comments expressed love and support towards them. So, those comments fall into positive sentiment by classification because of their positive words. This shows that even if the tweet and its' comments were showing opposite sentiment, they were actually agreeing on the tweet.

The idea for this research was to analyze Twitter network from a comparatively newer perspective. Users behavior were observed based on their text (tweets, replies) along with numerical scores like number of tweets, followers, likes, followees. All those values were combined with the sentiment and emotion of the users on certain topics to measure the effect. Existing researches focused on the text of the tweets where text from both tweets and replies were considered in this research to find the communication between users in more humane aspects. In most cases, the experiment found that users who reply to tweets normally share similar emotion or sentiment and agree to the tweet content. But there were cases where the reply opposed the original tweet emotion and expressed the disagreement of the replier very clearly. After considering all these factors to find influential users of a network, the result was more specific to a certain issue or topic. The experiment provided a general recommendation to show who are the most influential users expressing a certain emotion or sentiment on an issue. The personalized recommender provided customized recommendation to suggest from the network information about other people in a descending order of similarity who share similar sentiment or emotion on a topic as the user himself/herself. 


\subsection{Future Works}

Based on the study described in this research, a number of aspects were identified that were investigated further with the hope to produce an improved model and hence produce better results. For instance, one of the limitations of this work is that the system can not process tweets containing multiple emotions which can be considered as a possible future work. Detecting sarcasms, slangs, metaphors and implicit emotions can also be some possible fields to focus on in future. In this thesis, all emotions were considered with the same intensity which is not the case in real life. Assigning different weights to different emotions based on their intensity and the involved user would be interesting to investigate. A comparison between the classification accuracies of multiple supervised and unsupervised classifiers can also be a possible future work to detect the most appropriate classifier for the new dataset mentioned in chapter 4 and 5. Also, an automatic emotion and sentiment annotator using unsupervised classifier can accelerate the data preprocessing system.

Another possible topic to focus on in future can be the recommender system. The proposed approach is the first step towards a personalized recommender system for social networks. The system is static and is limited to the range of collected data within a limited time period and lots of missing information on users. A dynamic recommender system to provide suggestions based on any topic on any social network using user emotion and sentiment can be a possible future work which can use this framework to recommend various preferable topics to users. Weights were assigned to different user and tweet based parameters using AHP and the weights were static throughout the experiments. A dynamic weight generation system which can start from initial weight values and can update the weights using machine learning to achieve higher accuracy can be another possible topic for future implementations.

In spite of the use of different audio-visual technologies, people still use text in most of their interactions within their social networks and for their posting on social media forums. 
For a long time, various organizations related to business, psychology, health-care, politics, security and other fields have been trying to extract emotion of people from their social network interactions. Due to the necessity of detecting the correct emotion from a piece of text in various socio-economical areas, researchers have developed automatic emotion detection systems using different approaches. Researchers are still working on this topic to optimize existing systems and to increase their accuracy. Since it is nearly impossible to identify all variations of text representing all possible human emotions, the current research described in the literature has a wide range of possibilities for improvement. 


\section{Bibliography}

[1] Kashfia Sailunaz, Manmeet Dhaliwal, Jon Rokne, and Reda Alhajj. Emotion Detection from Text and Speech: A Survey. Social Network Analysis and Mining, Springer, 8(1):28, 2018.

[2] Kashfia Sailunaz, Tansel Özyer, Jon Rokne, and Reda Alhajj. Text-Based Analysis of Emotion by Considering Tweets. Machine Learning Techniques for Online Social Networks, Springer, pages 219-236, 2018.

[3] Roddy Cowie, Ellen Douglas-Cowie, Nicolas Tsapatsoulis, George Votsis, Stefanos Kollias, Winfried Fellenz, and John G. Taylor. Emotion Recognition in Human-Computer Interaction. IEEE Signal processing magazine, 18(1):32-80, 2001.

[4] Jesper Kjeldskov, and Connor Graham. A Review of Mobile HCI Research Methods. International Conference on Mobile Human-Computer Interaction, Springer, Berlin, Heidelberg, pages 317-335, 2003.

[5] Jonghwa Kim, Johannes Wagner, Thurid Vogt, Elisabeth André, Frank Jung, and Matthias Rehm. Emotional Sensitivity in Human-Computer Interaction. itInformation Technology Methoden und innovative Anwendungen der Informatik und Informationstechnik, 51(6):325-328, 2009.

[6] John F. Pane, Brad A. Myers, and Leah B. Miller. Using HCI Techniques to Design a more Usable Programming System. Human Centric Computing Languages and Environments, Proceedings. IEEE 2002 Symposia on, IEEE, pages 198-206, 2002. 
[7] Jerome R Bellegarda. Large-Scale Personal Assistant Technology Deployment: The Siri Experience. INTERSPEECH, pages 2029-2033, 2013.

[8] Alexander Maedche, Stefan Morana, Silvia Schacht, Dirk Werth, and Julian Krumeich. Advanced User Assistance Systems. Business Information Systems Engineering, 58(5):367-370, 2016.

[9] S. Lalitha, Sahruday Patnaik, T. H. Arvind, Vivek Madhusudhan, and Shikha Tripathi. Emotion Recognition through Speech Signal for Human-Computer Interaction. Electronic System Design (ISED), 2014 Fifth International Symposium on, IEEE, pages $217-218,2014$.

[10] Oh-Wook Kwon, Kwokleung Chan, Jiucang Hao, and Te-Won Lee. Emotion Recognition by Speech Signals. Eighth European Conference on Speech Communication and Technology, 2003.

[11] Thapanee Seehapoch, and Sartra Wongthanavasu. Speech Emotion Recognition using Support Vector Machines. Knowledge and Smart Technology (KST), 2013 5th International Conference on, IEEE, pages 86-91, 2013.

[12] Rafael A. Calvo, and Sunghwan Mac Kim. Emotions in Text: Dimensional and Categorical Models. Computational Intelligence, 29(3):527-543, 2013.

[13] Isidoros Perikos, and Ioannis Hatzilygeroudis. Recognizing Emotions in Text using Ensemble of Classifiers. Engineering Applications of Artificial Intelligence, 51:191$201,2016$.

[14] AFM Nazmul Haque Nahin, Jawad Mohammad Alam, Hasan Mahmud, and Kamrul Hasan. Identifying Emotion by Keystroke Dynamics and Text Pattern Analysis. Behaviour Information Technology, 33(9):987-996, 2014. 
[15] Saif M. Mohammad, and Svetlana Kiritchenko. Using Hashtags to Capture Fine Emotion Categories from Tweets. Computational Intelligence, 31(2):301-326, 2015.

[16] Ira Cohen, Ashutosh Garg, and Thomas S. Huang. Emotion Recognition from Facial Expressions using Multilevel HMM. Neural Information Processing Systems, 2, 2000.

[17] Mark Rosenblum, Yaser Yacoob, and Larry Davis. Human Emotion Recognition from Motion using a Radial basis Function Network Architecture. Motion of Non-Rigid and Articulated Objects 1994., Proceedings of the 1994 IEEE Workshop on, IEEE, pages 43-49, 1994.

[18] Debishree Dagar, Abir Hudait, H. K. Tripathy, and M. N. Das. Automatic Emotion Detection Model from Facial Expression. Advanced Communication Control and Computing Technologies (ICACCCT), 2016 International Conference on, IEEE, pages $77-85,2016$.

[19] Loic Kessous, Ginevra Castellano, and George Caridakis. Multimodal Emotion Recognition in Speech-based Interaction using Facial Expression, Body Gesture and Acoustic Analysis. Journal on Multimodal User Interfaces, 3(1-2):33-48, 2010.

[20] Jorg J. Schmitt, Wolfgang Hartje, and Klaus Willmes. Hemispheric Asymmetry in the Recognition of Emotional Attitude Conveyed by Facial Expression, Prosody and Propositional Speech. Cortex, 33(1):65-81, 1997.

[21] Amazon, https://www.amazon.ca/.

[22] Daniel Neiberg, Kjell Elenius, and Kornel Laskowski. Emotion Recognition in Spontaneous Speech using GMMs. Ninth International Conference on Spoken Language Processing, 2006. 
[23] Myriam D. Munezero, Calkin Suero Montero, Erkki Sutinen, and John Pajunen. Are they Different? Affect, Feeling, Emotion, Sentiment, and Opinion Detection in Text. IEEE Transactions on Affective Computing, 5(2):101-111, 2014.

[24] Oxford Living Dictionaries, https://en.oxforddictionaries.com/.

[25] Cambridge Dictionary, https://dictionary.cambridge.org/.

[26] American Psychological Association. Glossary of Psychological Terms, http://www.apa.org/research/action/glossary.aspx?tab=5.

[27] Charlie Dunbar Broad. Emotion and Sentiment. The Journal of Aesthetics and Art Criticism, 13(2):203-214, December 1954.

[28] Statista. Number of Social Media Users Worldwide from 2010 to 2021 (in billions), https://www.statista.com/statistics/278414/number-of-worldwide-socialnetwork-users/.

[29] Arthur Huang, David Ebert, and Parker Rider. You Are What You Tweet: A New Hybrid Model for Sentiment Analysis. International Conference on Machine Learning and Data Mining in Pattern Recognition, Springer, Cham, pages 403-416, 2017.

[30] Twitter, https://twitter.com/.

[31] Charles Darwin. The Expression of the Emotions in Man and Animals. Oxford University Press, USA, 1998.

[32] A. R. Manser. Sketch for a Theory of the Emotions. Analytic Philosophy, 4(1):27-28, January 1963.

[33] Charles Bell. Essays on the Anatomy of Expression in Painting. Essays on the Anatomy and Philosophy of Expression. 1824. 
[34] Joseph E LeDoux. Cognition and Emotion. Handbook of Cognitive Neuroscience, Springer US, pages 357-368, 1984.

[35] Joan C Borod. The Neuropsychology of Emotion. Oxford University Press, 2000.

[36] Sreeja PS, and G. S. Mahalakshmi. Emotion Models: A Review. International Journal of Control Theory and Applications (IJCTA), 10(8):651-657, 2017.

[37] Lea Canales, and Patricio Martinez-Barco. Emotion Detection from Text: A Survey. Processing in the 5th Information Systems Research Working Days (JISIC 2014), pages 37-43, 2014.

[38] P Ekman. An Argument for Basic Emotions. Cognition and emotion, 6(3-4):169-200, 1992.

[39] Phillip Shaver, Judith Schwartz, Donald Kirson, and Cary O'connor. Emotion Knowledge: Further Exploration of a Prototype Approach. Journal of Personality and Social Psychology, 52(6):1061-1086, 1987.

[40] Keith Oatley, and Philip N. Johnson-Laird. Towards a Cognitive Theory of Emotions. Cognition and Emotion, 1(1):29-50, 1987.

[41] Robert Plutchik. Emotion: A Psychoevolutionary Synthesis. Harper and Row, 1980.

[42] James A. Russell. A Circumplex Model of Affect. Journal of Personality and Social Psychology, 39(6):1161-1178, 1980.

[43] Gerald L. Clore Andrew Ortony and Allan Collins. The Cognitive Structure of Emotions. Cambridge University Press, 1988.

[44] Hugo Lovheim. A New Three-Dimensional Model for Emotions and Monoamine Neurotransmitters. Medical Hypotheses, 78(2):341-348, 2012. 
[45] Soujanya Poria, Iti Chaturvedi, Erik Cambria, and Amir Hussain. Convolutional MKL based Multimodal Emotion Recognition and Sentiment Analysis. Data Mining (ICDM), 2016 IEEE 16th International Conference on, pages 439-448, 2016.

[46] Krishna Mohan Kudiri, Abas Md Said, and M. Yunus Nayan. Human Emotion Detection through Speech and Facial Expressions. Computer and Information Sciences (ICCOINS), 2016 3rd International Conference on, IEEE, pages 351-356, 2016.

[47] Mohammad Soleymani, Sadjad Asghari-Esfeden, Yun Fu, and Maja Pantic. Analysis of EEG Signals and Facial Expressions for Continuous Emotion Detection. IEEE Transactions on Affective Computing, 7(1):17-28, 2016.

[48] Nancy Semwal, Abhijeet Kumar, and Sakthivel Narayanan. Automatic Speech Emotion Detection System using Multi-Domain Acoustic Feature Selection and Classification Models. Identity, Security and Behavior Analysis (ISBA), 2017 IEEE International Conference on, IEEE, pages 1-6, 2017.

[49] Christos-Nikolaos Anagnostopoulos, Theodoros Iliou, and Ioannis Giannoukos. Features and Classifiers for Emotion Recognition from Speech: A Survey from 2000 to 2011. Artificial Intelligence Review, 43(2):155-177, 2015.

[50] Samira Ebrahimi Kahou, Xavier Bouthillier, Pascal Lamblin, Caglar Gulcehre, Vincent Michalski, Kishore Konda, Sebastien Jean, Pierre Froumenty, Yann Dauphin, Nicolas Boulanger-Lewandowski, Raul Chandias Ferrari, Mehdi Mirza, David Warde-Farley, Aaron Courville, Pascal Vincent, Roland Memisevic, Christopher Pal, and Yoshua Bengio. EmoNets: Multimodal Deep Learning Approaches for Emotion Recognition in Video. Journal on Multimodal User Interfaces, 10(2):99-111, 2016.

[51] Ali Yadollahi, Ameneh Gholipour Shahraki, and Osmar R. Zaiane. Current State of Text Sentiment Analysis from Opinion to Emotion Mining. ACM Computing Surveys (CSUR), 50(2):25:1-25:33, 2017. 
[52] Chetan R. Chopade. Text Based Emotion Recognition: A Survey. International Journal of Science and Research (IJSR), 4(6):409-414, 2015.

[53] Vaibhav Tripathi, Aditya Joshi, and Pushpak Bhattacharyya. Emotion Analysis from Text: A Survey. http://www.cfilt.iitb.ac.in/resources/surveys/emotion-analysissurvey-2016-vaibhav.pdf.

[54] Haji Binali, Chen Wu, and Vidyasagar Potdar. Computational Approaches for Emotion Detection in Text. Digital Ecosystems and Technologies (DEST), 2010 4th IEEE International Conference on, pages 172-177, 2010.

[55] Edward Chao-Chun Kao, Chun-Chieh Liu, Ting-Hao Yang, Chang-Tai Hsieh, and Von-Wun Soo. Towards Text-Based Emotion Detection a Survey and Possible Improvements. Information Management and Engineering, 2009. ICIME'09. International Conference on, IEEE, pages 70-74, 2009.

[56] Shiv Naresh Shivhare, and Saritha Khethawat. Emotion Detection from Text. 2012.

[57] Umang Gupta, Ankush Chatterjee, Radhakrishnan Srikanth, and Puneet Agrawal. A Sentiment-and-Semantics-Based Approach for Emotion Detection in Textual Conversations. Neu-IR: Workshop on Neural Information Retrieval, SIGIR 2017, ACM, arXiv preprint arXiv:1707.06996., 2017.

[58] Bart Desmet, and VeRonique Hoste. Emotion Detection in Suicide Notes. Expert Systems with Applications, 40(16):6351-6358, 2013.

[59] Luca Dini, and Andre Bittar. Emotion Analysis on Twitter: The Hidden Challenge. Language Resources and Evaluation Conference (LREC), 2016.

[60] Saif M. Mohammad, and Felipe Bravo-Marquez. Emotion Intensities in Tweets. Proceedings of the Sixth Joint Conference on Lexical and Computational Semantics ( ${ }^{*}$ Sem), 2017. 
[61] Anja Summa, Bernd Resch, Geoinformatics-Z. GIS, and Michael Strube. Microblog Emotion Classification by Computing Similarity in Text, Time, and Space. Proceedings of the Workshop on Computational Modeling of People's Opinions, Personality, and Emotions in Social Media, pages 153-162, 2016.

[62] Anirban Sen, Manjira Sinha, Sandya Mannarswamy, and Shourya Roy. Multi-Task Representation Learning for Enhanced Emotion Categorization in Short Text. PacificAsia Conference on Knowledge Discovery and Data Mining, Springer, Cham, pages 324-336, 2017.

[63] Vinay Kumar Jain, Shishir Kumar, and Steven Lawrence Fernandes. Extraction of Emotions from Multilingual Text using Intelligent Text Processing and Computational Linguistics. Journal of Computational Science, 2017.

[64] Xin Kang, Fuji Ren, and Yunong Wu. Exploring Latent Semantic Information for Textual Emotion Recognition in Blog Articles. IEEE/CAA Journal of Automatica Sinica, 2017.

[65] Ameeta Agrawal, and Aijun An. Unsupervised Emotion Detection from Text using Semantic and Syntactic Relations. Proceedings of the The 2012 IEEE/WIC/ACM International Joint Conferences on Web Intelligence and Intelligent Agent Technology, IEEE Computer Society, 1:346-353, 2012.

[66] Sudhanshu Prakash Tiwari, M. Vijaya Raju, Gurbakash Phonsa, and Deepak Kumar Deepu. A Novel Approach for Detecting Emotion in Text. Indian Journal of Science and Technology, 9(29), 2016.

[67] Sophia Yat Mei Lee, Ying Chen, and Chu-Ren Huang. A Text-Driven Rule-Based System for Emotion Cause Detection. Proceedings of the NAACL HLT 2010 Workshop on Computational Approaches to Analysis and Generation of Emotion in Text, Association for Computational Linguistics, pages 45-53, 2010. 
[68] Weiyuan Li, and Hua Xu. Text-Based Emotion Classification using Emotion Cause Extraction. Expert Systems with Applications, 41(4):1742-1749, 2014.

[69] Yunjing An, Shutao Sun, and Shujuan Wang. Naive Bayes Classifiers for Music Emotion Classification based on Lyrics. Computer and Information Science (ICIS), 2017 IEEE/ACIS 16th International Conference on, pages 635-638, 2017.

[70] Mousannif Hajar. Using YouTube Comments for Text-based Emotion Recognition. Procedia Computer Science, 83:292-299, 2016.

[71] Xiangsheng Li, Jianhui Pang, Biyun Mo, and Yanghui Rao. Hybrid Neural Networks for Social Emotion Detection Over Short Text. Neural Networks (IJCNN), 2016 International Joint Conference on, IEEE, pages 537-544, 2016.

[72] Carlo Strapparava, and Rada Mihalcea. Learning to Identify Emotions in Text. Proceedings of the 2008 ACM symposium on Applied computing, ACM, pages 1556-1560, 2008.

[73] Diman Ghazi, Diana Inkpen, and Stan Szpakowicz. Prior and Contextual Emotion of Words in Sentential Context. Computer Speech Language, 28(1):76-92, 2014.

[74] Maryam Hasan, Elke Rundensteiner, Xiangnan Kong, and Emmanuel Agu. Using Social Sensing to Discover Trends in Public Emotion. Semantic Computing (ICSC), 2017 IEEE 11th International Conference on,IEEE, pages 172-179, 2017.

[75] Maryam Hasan, Elke Rundensteiner, and Emmanuel Agu. Emotex: Detecting Emotions in Twitter Messages. 2014 ASE BIGDATA/SOCIALCOM/CYBERSECURITY Conference, 2014.

[76] Neeraj Kanger, and Gourav Bathla. Recognizing Emotion in Text using Neural Network and Fuzzy Logic. Indian Journal of Science and Technology, 10(12), 2017. 
[77] Sheeba Grover, and Amandeep Verma. Design for Emotion Detection of Punjabi Text using Hybrid Approach. Inventive Computation Technologies (ICICT), International Conference on, IEEE, 2:1-6, 2016.

[78] Aditya Joshi, Vaibhav Tripathi, Ravindra Soni, Pushpak Bhattacharyya, and Mark James Carman. EmoGram: An Open-Source Time Sequence-Based Emotion Tracker and Its Innovative Applications. AAAI Workshop: Knowledge Extraction from Text, 2016.

[79] JULIELab. EmoBank, https://github.com/JULIELab/EmoBank.

[80] Preotiuc-Pietro and Others. The Valence and Arousal Facebook Posts, http://wwbp.org/downloads/public ${ }_{d}$ ata/dataset - fb-valence-arousal-anon.csv.

[81] CrowdFlower. The Emotion in Text data set, https://www.crowdflower.com/wpcontent/uploads/2016/07/text motion.csv.

[82] Saif Mohammad and Peter Turney. NRC Word-Emotion Association Lexicon, http://www.saifmohammad.com/WebPages/NRC-Emotion-Lexicon.htm.

[83] AAAC Emotion Research. ISEAR Databank, http://emotionresearch.net/toolbox/toolboxdatabase.2006-10-13.2581092615.

[84] Carlo Strapparava, and Rada Mihalcea. SemEval-2007 Task 14: Affective Text, http://web.eecs.umich.edu/ mihalcea/affectivetext/.

[85] Haewoon Kwak, Changhyun Lee, Hosung Park, and Sue Moon. What is Twitter, a Social Network or a News Media? Proceedings of the 19th International Conference on World Wide Web, ACM, pages 591-600, 2010.

[86] Soroush Vosoughi, Deb Roy, and Sinan Aral. The Spread of True and False News Online. Science, 359(6380):1146-1151, 2018. 
[87] Fabian Riquelme, and Pablo Gonzalez-Cantergiani. Measuring User Influence on Twitter: A Survey. Information Processing Management, 52(5):949-975, 2016.

[88] Eleanna Kafeza, Andreas Kanavos, Christos Makris, and Pantelis Vikatos. T-PICE: Twitter Personality based Influential Communities Extraction System. Big Data (BigData Congress), 2014 IEEE International Congress on, IEEE, pages 212-219, 2014.

[89] Chiara Francalanci, and Ajaz Hussain. Influence-based Twitter Browsing with NavigTweet. Information Systems, 64:119-131, 2017.

[90] Eva Lahuerta-Otero, and Rebeca Cordero-Gutierrez. Looking for the Perfect Tweet. The Use of Data Mining Techniques to Find Influencers on Twitter. Computers in Human Behavior, 64:575-583, 2016.

[91] Lorenzo Coviello, Yunkyu Sohn, Adam DI Kramer, Cameron Marlow, Massimo Franceschetti, Nicholas A. Christakis, and James H. Fowler. Detecting Emotional Contagion in Massive Social Networks. PloS One, 9(3):e90315, 2014.

[92] Iris Roelens, Philippe Baecke, and Dries F. Benoit. Identifying Influencers in a Social Network: The Value of Real Referral Data. Decision Support Systems, 91:25-36, 2016.

[93] Rinkesh Nagmoti, Ankur Teredesai, and Martine De Cock. Ranking Approaches for Microblog Search. Web Intelligence and Intelligent Agent Technology (WI-IAT), 2010 IEEE/WIC/ACM International Conference on, 1:153-157, 2010.

[94] Tomoya Noro, Fei Ru, Feng Xiao, and Takehiro Tokuda. Twitter User Rank using Keyword Search. Information Modelling and Knowledge Bases XXIV. Frontiers in Artificial Intelligence and Applications, 251:31-48, 2013.

[95] Aditya Pal, and Scott Counts. Identifying Topical Authorities in Microblogs. Proceedings of the Fourth ACM International Conference on Web Search and Data Mining, pages 45-54, 2011. 
[96] Carolina Bigonha, Thiago NC Cardoso, Mirella M. Moro, Virgílio AF Almeida, and Marcos A. Gonçalves. Detecting Evangelists and Detractors on Twitter. 18th Brazilian Symposium on Multimedia and the Web, pages 107-114, 2010.

[97] Daniel Gayo-Avello, David J. Brenes, Diego Fernández-Fernández, María E. Fernández-Menéndez, and Rodrigo García-Suárez. De Retibus Socialibus et Legibus Momenti. EPL (Europhysics Letters), 94(3):38001, 2011.

[98] M. S. Srinivasan, Srinath Srinivasa, and Sunil Thulasidasan. Exploring Celebrity Dynamics on Twitter. Proceedings of the 5th IBM Collaborative Academia Research Exchange Workshop, ACM, page 13, 2017.

[99] Vito Latora, and Massimo Marchiori. A Measure of Centrality based on Network Efficiency. New Journal of Physics, 9(6), 2007.

[100] Jorge E Hirsch. An Index to Quantify an Individual's Scientific Research Output that Takes into Account the Effect of Multiple Coauthorship. Scientometrics, 85(3):741$754,2010$.

[101] Daniel M. Romero, Wojciech Galuba, Sitaram Asur, and Bernardo A. Huberman. Influence and Passivity in Social Media. Joint European Conference on Machine Learning and Knowledge Discovery in Databases, Springer, Berlin, Heidelberg, pages 18-33, 2011.

[102] Daniel Tunkelang. A Twitter analog to Pagerank, http://thenoisychannel.com/2009/01/13/a-twitter-analog-to-pagerank.

[103] Tomáš Majer, and Marián Šimko. Leveraging Microblogs for Resource Ranking. International Conference on Current Trends in Theory and Practice of Computer Science, Springer, Berlin, Heidelberg, pages 518-529, 2012. 
[104] Abolfazl Aleahmad, Payam Karisani, Maseud Rahgozar, and Farhad Oroumchian. OLFinder: Finding Opinion Leaders in Online Social Networks. Journal of Information Science, 42(5):659-674, 2016.

[105] Your Dictionary. Examples of Sarcasm, http://examples.yourdictionary.com/examplesof-sarcasm.html.

[106] Wordnet, https://wordnet.princeton.edu/.

[107] Dictionary.com, http://www.dictionary.com/.

[108] Thesaurus.com, http://www.thesaurus.com/.

[109] Wordsmyth, http://www.wordsmyth.net/.

[110] Twittonary, http://twittonary.com/.

[111] Twictionary, http://twictionary.pbworks.com/w/page/22547584/frontpage.

[112] Data for everyone, https://www.crowdflower.com/data-for-everyone/.

[113] Statista. Number of Monthly Active Twitter Users Worldwide from 1st Quarter 2010 to 4th Quarter 2017 (in millions).

[114] Thomas L Saaty. How to Make a Decision: The Analytic Hierarchy Process. European Journal of Operational Research, 48(1):9-26, 1990. 